FACULDADE DE MEDICINA DA UNIVERSIDADE DE BRASÍLIA PROGRAMA DE PÓS-GRADUAÇÃO EM CIÊNCIAS MÉDICAS

CARLOS JANSSEN GOMES

DISSERTAÇÃO DE MESTRADO

Brasília 


\section{DINÂMICA CRONOTRÓPICA PÓS-ESFORÇO E FUNÇÃO AUTONÔMICA CARDÍACA DE REPOUSO EM PRATICANTES DE DANÇA DE SALÃO}

Dissertação de mestrado
apresentada ao Programa de Pós-
Graduação em Ciências Médicas
da Faculdade de Medicina, como
requisito para a obtenção do título
de Mestre em Ciências Médicas -
Área de concentração: Ciências
Aplicadas em Saúde.

Aluno: Carlos Janssen Gomes

Orientador: Prof. Dr. Luiz Fernando Junqueira Jr.

Co-orientador: Prof. Dr. Guilherme Eckhardt Molina

Brasília 


\section{DEDICATÓRIA}

Durante toda a minha trajetória acadêmica, diversas pessoas contribuíram das mais variadas formas para a realização de um sonho, o ingresso em um programa de mestrado. Hoje, como egresso, agradeço à Deus e dedico essa obra à todos os que fizeram parte da minha jornada acadêmica/profissional. Em destaque, agradeço e dedico esse manuscrito à senhora Ilma Pires Gomes, amiga, mãe e companheira de todos os momentos. 


\section{Agradecimentos}

Aos familiares e amigos, que sempre respeitam e apoiam minhas decisões. Em especial aos meus filhos mais velhos Marcos Adiel, Lorena Pires e Ludmila Fernanda, e aos pequeninos, João Gabriel, Carla, Isadora e Juliana. Se amor e educação são critérios para ser "pai", todos vocês são meus filhos.

À senhora Ilma Pires Gomes, minha mãe, por toda a rigorosa criação proporcionada. Mesmo diante de todas as dificuldades que enfrentou ao longo de sua vida, sempre fez questão de nos proporcionar uma criação baseada no respeito, humildade e muito amor (também nos apresentava o cinto quando necessário...). Amo você "magrela"!

Aos voluntários da pesquisa e aos técnicos do Laboratório de Avaliação do Desempenho Físico e da Saúde (LADEFS-UNIEURO), que sempre deram todo o suporte necessário para a realização do presente trabalho.

Ao professor Dr. Luiz Guilherme Grossi Porto, que de maneira muito agradável, humilde e respeitosa prestou relevantes contribuições à minha formação acadêmica, além de proporcionar diversos momentos de descontração e boas gargalhadas em nossos encontros no "quintal do prof. Junqueira".

Ao professor Dr. Guilherme Eckhardt Molina pelos valiosos cinco anos de parceria e amizade. Sempre será mencionado como um exemplo que procuro seguir a "margens estreitas" desde a primeira aula assistida. Muito obrigado por toda formação proporcionada, pelo laço de amizade que se torna cada dia mais forte, pelos sábios conselhos e por conduzir com maestria minha vida acadêmica. Esse mestrado é nosso!

Ao professor Dr. Luiz Fernando Junqueira Jr, por ter concedido a honra de sua orientação, por todos os "desconfortos intelectuais" provocados a cada reunião e por mostrar que um pesquisador deve estar além da fisiologia o dos valores de " $p$ ". Espero estar suprindo as expectativas quanto orientando, agora eu acredito que "está pronto". Muito obrigado! 


\section{Sumário}

Resumo

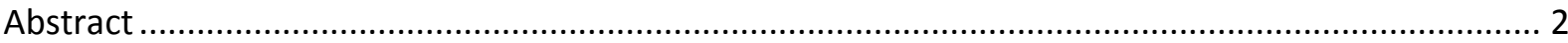

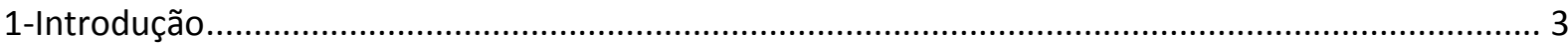

1.1-Visão geral do sistema cardiovascular e sua regulação .............................................................. 5

1.2-Avaliação clínica da função autonômica cardíaca .................................................................. 9

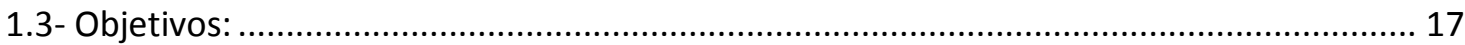

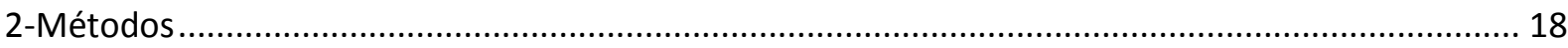

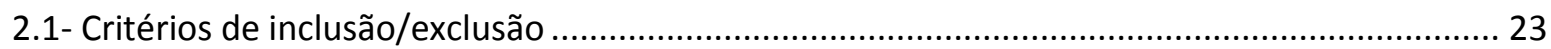

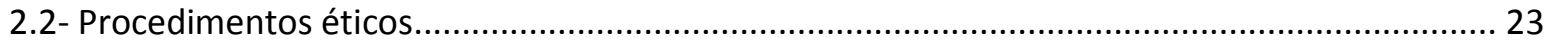

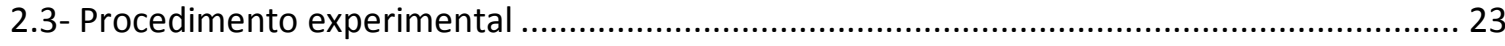

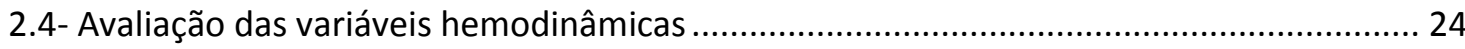

2.5- Avaliação da função autonômica cardíaca.................................................................... 25

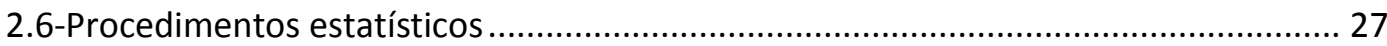

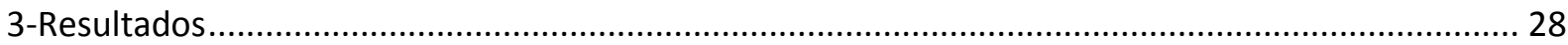

3.1- Análise cronotrópica antes, durante e após o teste de esforço submáximo............................ 28

3.2- Análise da variabilidade da frequência cardíaca nas condições de repouso, durante o teste de esforço e no período de recuperação após do teste de esforço submáximo.

3.3-Correlação entre a variabilidade da frequência cardíaca e o decremento da frequência cardíaca

3.4-Correlação entre a variabilidade da frequência cardíaca em repouso e o coeficiente de

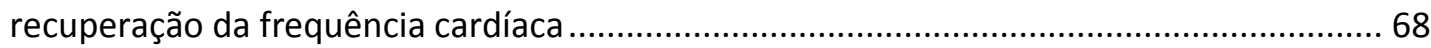

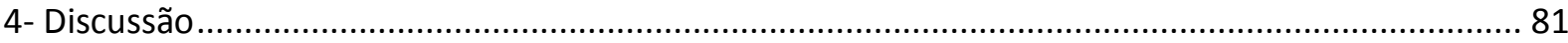

4.1- Análise cronotrópica antes, durante e após o teste de esforço submáximo ............................. 81

4.2- Análise da variabilidade da frequência cardíaca em condição de repouso ............................. 85

4.3-Análise da variabilidade da frequência cardíaca durante e após o teste de esforço submáximo

4.4-Correlação entre a variabilidade da frequência cardíaca de repouso e o decremento absoluto da frequência cardíaca após o teste de esforço

4.5-Correlação entre a variabilidade da frequência cardíaca em repouso e o coeficiente de recuperação da frequência cardíaca ...................................................................... 92

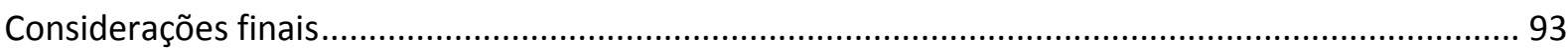

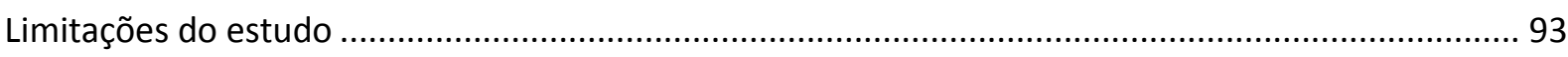

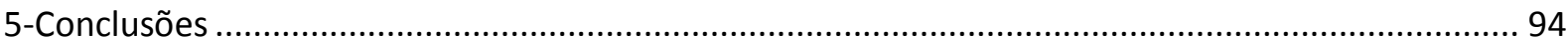


6-Referências bibliográficas 96

ANEXOS 110

ANEXO I: Termo de consentimento livre e esclarecido 110

ANEXO II: Parecer do comitê de ética..... 112

ANEXO III: Dados individuais da frequência cardíaca e sua variabilidade nos grupos controle e praticantes de dança

Erro! Indicador não definido.

ANEXO IV: Análise de correlação entre a variabilidade da frequência cardíaca em repouso e o decremento relativo da frequência cardíaca 


\section{Lista de abreviaturas}

1. AF- Área espectral de alta frequência

2. BF- Área espectral de baixa frequência

3. CCC-Centro de controle cardiovascular

4. CR-Coeficiente de recuperação

5. CT-Grupo controle

6. CV-Coeficiente de variação

7. DFC-Decremento absoluto da frequência cardíaca

8. DFC\%-Decremento relativo da frequência cardíaca

9. DP- Desvio parão

10. FAC- Função autonômica cardíaca

11. FC- Frequência cardíaca

12. FC $_{\text {sup }}$ - Frequência cardíaca na posição supina

13. FC $_{\text {ort- }}$ Frequência cardíaca na posição ortostática

14. $\mathbf{F C}_{\text {pico- }}$-Frequência cardíaca registrada ao final do teste de esforço

15. FR- Frequência respiratória

16. IMC- Índice de massa corporal

17. iRR- Intervalo R-R

18. Kg- Quilograma

19. Km/h- Quilômetro por hora

20. LVFC- Limiar de variabilidade da frequência cardíaca

21. NTS- Núcleo do trato solitário

22. PAS- Pressão arterial sistólica

23. PAD- Pressão arterial diastólica

24. pNN50- percentual de intervalos R-R adjacentes com diferença superior a $50 \mathrm{~ms}$

25. Razão BF/AF- Razão entre a área absoluta de baixa frequência pela área espectral absoluta de alta frequência

26. Razão SD1/SD2- Razão entre o índice SD1 pelo índice SD2

27. RC- Reserva cronotrópica

28. r-MSSD- raiz quadrada da média do quadrado das diferenças sucessivas dos intervalos R-R

29. $\mathbf{r}_{\mathrm{s}}$ - Correlação de Spearman

30. SNA- Sistema nervoso autônomo 
31. TES- Teste de esforço submáximo

32. VD- Velocidade do decremento da frequência cardíaca

33. VFC- Variabilidade da frequência cardíaca

\section{Lista de figuras}

Figura 1- Representação da análise espectral .12

Figura 2- Representação da plotagem de Poincaré..... .13

Figura 3- Representação do limiar de variabilidade da frequência cardíaca. 14

Figura 4- Esquema temporal do registro das variáveis hemodinâmicas. .24

\section{Lista de tabelas}

Tabela 1.1- Identificação, ocupação e valores individuais das características dos voluntários inseridos no grupo controle.

Tabela 1.2: Identificação, ocupação e valores individuais das características dos voluntários inseridos no grupo de praticantes de dança de salão .20

Tabela 1.3: Estatística descritiva amostral da idade e características antropométricas dos grupos controle e praticantes de dança de salão. .21

Tabela 1.4: Estatística descritiva amostral das características fisiológicas dos grupos controle e praticantes de dança de salão. .22

Tabela 2.1: Estatística descritiva amostral dos dados cronotrópicos registrados imediatamente antes e durante o teste de esforço submáximo comparativamente entre os grupos controle e praticantes de dança de salão 30

Tabela 2.2: Estatística descritiva amostral do decremento absoluto da frequência cardíaca após o teste de esforço submáximo comparativamente entre os grupos controle e praticantes de dança de salão.

Tabela 2.3: Estatística descritiva amostral do decremento relativo da frequência cardíaca após o teste de esforço submáximo comparativamente entre os grupos controle e praticantes de dança de salão. 
Tabela 2.4: Estatística descritiva amostral da velocidade do decremento da frequência cardíaca após o teste de esforço submáximo comparativamente entre os grupos controle e praticantes de dança de salão

Tabela 2.5: Estatística descritiva amostral do coeficiente de recuperação da frequência cardíaca após o teste de esforço submáximo comparativamente entre os grupos controle e praticantes de dança de salão

Tabela 3.1: Estatística descritiva amostral dos índices temporais da variabilidade da frequência cardíaca durante 5 minutos na posição supina, comparativamente entre os grupos e praticantes de dança de salão.

Tabela 3.2: Estatística descritiva amostral dos índices temporais da variabilidade da frequência cardíaca durante 5 minutos na posição ortostática, comparativamente entre os grupos controle e praticantes de dança de salão. .38

Tabela 3.3: Estatística descritiva amostral da variação relativa dos índices temporais da variabilidade da frequência cardíaca na transição da posição supina para posição ortostática, comparativamente entre os grupos controle e praticantes de dança de salão

Tabela 4.1: Estatística descritiva amostral dos índices espectrais da variabilidade da frequência cardíaca durante 5 minutos na posição supina, comparativamente entre os grupos controle e praticantes de dança de salão

Tabela 4.2: Estatística descritiva amostral dos índices espectrais da variabilidade da frequência cardíaca durante 5 minutos na posição ortostática, comparativamente entre os grupos controle e praticantes de dança de salão.

Tabela 4.3: Estatística descritiva amostral da variação relativa dos índices espectrais da variabilidade da frequência cardíaca na transição da posição supina para posição ortostática, comparativamente entre os grupos controle e praticantes de dança de salão

Tabela 5.1: Estatística descritiva amostral dos índices tempo-frequenciais da variabilidade da frequência cardíaca durante 5 minutos na posição supina, comparativamente entre os grupos controle e praticantes de dança de salão.

Tabela 5.2: Estatística descritiva amostral dos índices tempo-frequenciais da variabilidade da frequência cardíaca durante 5 minutos na posição ortostática, comparativamente entre os grupos controle e praticantes de dança de salão. 
Tabela 5.3: Estatística descritiva amostral da variação relativa dos índices tempo-frequenciais da variabilidade da frequência cardíaca na transição da posição supina para posição ortostática, comparativamente entre os grupos controle e praticantes de dança de salão. .45

Tabela 6.1: Estatística descritiva amostral dos índices do Poincaré da variabilidade da frequência cardíaca durante 5 minutos na posição supina, comparativamente entre os grupos e praticantes de dança de salão.

Tabela 6.2: Estatística descritiva amostral dos índices de Poincaré durante 5 minutos na posição ortostática, comparativamente entre os grupos controle e praticantes de dança de salão...

Tabela 6.3: Estatística descritiva amostral da variação relativa dos índices de Poincaré na transição da posição supina para posição ortostática, comparativamente entre os grupos controle e praticantes de dança de salão.

Tabela 6.4: Proporção de praticantes de dança que apresentaram índices de variabilidade da frequência cardíaca dentro, acima ou abaixo da amplitude interquartílica considerando os registros do grupo controle.

Tabela 6.5: Estatística descritiva amostral de todos os índices que apresentaram diferenças entre os grupos controle e praticantes de dança nas posições supina, ortostática e na variação com a transição da posição supina para posição ortostática

Tabela 7.1: Estatística descritiva amostral dos índices tempo-frequenciais da variabilidade da frequência cardíaca registrados no período 5minutos de recuperação após o teste de esforço, comparativamente entre os grupos controle e praticantes de dança de salão. .52

Tabela 7.2: Estatística descritiva amostral dos índices de Poincaré registrados no período de 5minutos de recuperação após o teste de esforço, comparativamente entre os grupos controle e praticantes de dança de salão. . .53

Tabela 8.1: Correlação $(n=46)$ entre os diversos índices temporais das séries de intervalo RR na posição supina previamente ao esforço com o decremento absoluto das frequências cardíacas obtidas no período de recuperação ativa após o esforço. .56

Tabela 8.2: Correlação $(n=46)$ entre os diversos índices temporais das séries de intervalo RR na posição ortostática previamente ao esforço com o decremento 
absoluto das frequências cardíacas obtidas no período de recuperação ativa após o esforço

Tabela 8.3: Correlação $(n=46)$ entre a variação relativa dos diversos índices temporais das séries de intervalo RR na transição da posição supina para posição ortostática e o decremento absoluto das frequências cardíacas obtidas no período de recuperação ativa após o esforço. .58

Tabela 9.1: Correlação $(n=46)$ entre os diversos índices espectrais das séries de intervalo RR na posição supina previamente ao esforço com o decremento absoluto das frequências cardíacas obtidas no período de recuperação ativa após o esforço.

Tabela 9.2: Correlação $(\mathrm{n}=46)$ entre os diversos índices espectrais das séries de intervalo RR na posição ortostática previamente ao esforço com o decremento absoluto das frequências cardíacas obtidas no período de recuperação ativa após o esforço

Tabela 9.3: Correlação $(n=46)$ entre a variação relativa dos diversos índices espectrais das séries de intervalo RR na transição da posição supina para posição ortostática e o decremento absoluto das frequências cardíacas obtidas no período de recuperação ativa após o esforço.

Tabela 10.1: Correlação $(n=46)$ entre os diversos índices tempo-frequenciais das séries de intervalo RR na posição supina previamente ao esforço com o decremento absoluto das frequências cardíacas obtidas no período de recuperação ativa após o esforço 62

Tabela 10.2: Correlação $(n=46)$ entre os diversos índices tempo-frequenciais das séries de intervalo RR na posição ortostática previamente ao esforço com $o$ decremento absoluto das frequências cardíacas obtidas no período de recuperação ativa após o esforço.

Tabela 10.3: Correlação $(n=46)$ entre os diversos índices tempo-frequenciais das séries de intervalo $R R$ na transição da posição supina para posição ortostática previamente ao esforço e o decremento absoluto das frequências cardíacas obtidas no período de recuperação ativa após o esforço 
Tabela 11.1: Correlação $(n=46)$ entre os diversos índices de Poincaré na posição supina previamente ao esforço com o decremento absoluto das frequências cardíacas obtidas no período de recuperação ativa após o esforço. .65

Tabela 11.2: Correlação $(n=46)$ entre os diversos índices de Poincaré na posição ortostática previamente ao esforço com o decremento absoluto das frequências cardíacas obtidas no período de recuperação ativa após o esforço .66

Tabela 11.3: Correlação $(n=46)$ entre os diversos índices de Poincaré na transição da posição supina para posição ortostática previamente ao esforço e o decremento absoluto das frequências cardíacas obtidas no período de recuperação ativa após o esforço

Tabela 12.1: Correlação $(n=46)$ entre os diversos índices temporais das séries de intervalos RR na posição supina previamente ao esforço com o coeficiente de recuperação da frequência cardíaca.

Tabela 12.2: Correlação $(n=46)$ entre os diversos índices temporais das séries de intervalos RR na posição ortostática previamente ao esforço e o coeficiente de recuperação da frequência cardíaca. .70

Tabela 12.3: Correlação $(n=46)$ entre os diversos índices temporais das séries de intervalos RR na transição da posição supina para posição ortostática previamente ao esforço e o coeficiente de recuperação da frequência cardíaca. .71

Tabela 13.1: Correlação $(n=46)$ entre os diversos índices espectrais das séries de intervalos RR na posição supina previamente ao esforço e o coeficiente de recuperação da frequência cardíaca. .72

Tabela 13.2: Correlação $(n=46)$ entre os diversos índices espectrais das séries de intervalos RR na posição ortostática previamente ao esforço e o coeficiente de recuperação da frequência cardíaca.

Tabela 13.3: Correlação $(n=46)$ entre os diversos índices espectrais das séries de intervalos RR na transição da posição supina para posição ortostática previamente ao esforço e o coeficiente de recuperação da frequência cardíaca. 
Tabela 14.1: Correlação $(n=46)$ entre os diversos índices tempo-frequenciais das séries de intervalos RR na posição supina previamente ao esforço e o coeficiente de recuperação da frequência cardíaca.

Tabela 14.2: Correlação $(n=46)$ entre os diversos índices tempo-frequenciais das séries de intervalos RR na posição ortostática previamente ao esforço e o coeficiente de recuperação da frequência cardíaca.

Tabela 14.3: Correlação $(n=46)$ entre os diversos índices tempo-frequenciais das séries de intervalos $\mathrm{RR}$ na transição da posição supina para posição ortostática previamente ao esforço e o coeficiente de recuperação da frequência cardíaca.

Tabela 15.1: Correlação $(n=46)$ entre os diversos índices de Poincaré na posição supina previamente ao esforço e o coeficiente de recuperação da frequência cardíaca

Tabela 15.2: Correlação $(n=46)$ entre os diversos índices de Poincaré na posição ortostática previamente ao esforço e o coeficiente de recuperação da frequência cardíaca.

Tabela 15.3: Correlação $(n=46)$ entre os diversos índices de Poincaré na transição da posição supina para posição ortostática previamente ao esforço e o coeficiente de recuperação da frequência cardíaca. 


\section{Resumo}

Introdução: A prática de dança de salão, devido à sua característica lúdica e social, tem sido considerada uma importante estratégia na árdua tarefa de combate ao sedentarismo, um importante fator de risco associado ao surgimento de doenças cardiovasculares e à mortalidade por todas as causas. Por outro lado, ainda é obscuro se indivíduos envolvidos com a prática recreacional da dança, onde o controle da intensidade durante as sessões é negligenciada, apresentam um perfil cardiovascular mais favorável quando comparados a indivíduos sedentários ou insuficientemente ativos. Objetivos: Proceder a avaliação do comportamento da frequência cardíaca (FC) e da sua variabilidade (VFC) em praticantes de dança de salão (PD) comparativamente a indivíduos sedentários ou insuficientemente ativos (CT). Métodos: 50 voluntários do sexo masculino $(C T=25 ; P D=25)$ (Idade: $C T=25,9 \pm 4,5$; $\mathrm{PD}=26,6 \pm 6,1$ anos; IMC: $\mathrm{CT}=24,3 \pm 2,4 ; \mathrm{PD}=24,3 \pm 2,2 \mathrm{~kg} / \mathrm{m}^{2}$ ), foram submetidos à análise da frequência cardíaca (FC) e de sua variabilidade nas condições de repouso (posições supina e ortostática), durante teste de esforço submáximo e no período de recuperação após esforço. Resultados: O grupo PD apresentou menores valores de FC nas posições supina ( $\mathrm{p}=$ $0,001)$, ortostática $(p=0,05)$ e no estágio inicial do teste de esforço $(p=0,003)$, maior tolerância ao esforço medida pelo tempo de teste $(\mathrm{p}=0,0001)$ e maior decremento da frequência cardíaca no primeiro $(\mathrm{p}=0,007)$, segundo $(\mathrm{p}=0005)$, terceiro $(\mathrm{p}=0,0001)$, quarto $(\mathrm{p}=0,005)$ e quinto $(\mathrm{p}=0,01)$ minutos de recuperação. Quanto à análise da VFC nas três condições avaliadas verificou - se indícios de maior modulação da função autonômica cardíaca no grupo PD comparativamente ao CT na condição de repouso (supino e ortostático). No esforço, o limiar de VFC ocorreu tardiamente no grupo PD comparativamente ao CT ( $\mathrm{p}=$ 0,0001). Na recuperação, verificou-se maior grau de modulação autonômica cardíaca no período de recuperação no grupo PD comparativamente ao CT ( $\mathrm{p}=0.01-0.04)$. Conclusão: Os praticantes de dança de salão avaliados no presente estudo apresentaram um cronotropismo mais lento em relação ao grupo controle em todas as condições funcionais avaliadas. Adicionalmente, foram observados fortes indícios de que esse grupo também seja contemplado com um maior grau de modulação autonômica cardíaca nas condições de repouso, esforço e recuperação após esforço.

Palavras chave: Sistema nervoso autônomo, variabilidade da frequência cardíaca, exercício físico e dança de salão 


\section{Abstract}

Introduction: Ballroom dance practice, due to his playful and social characteristic, has been considered an important strategy in the arduous task of combating sedentary lifestyle, an important risk factor associated with the development of cardiovascular disease and mortality for all causes. On the other hand, it is still unclear whether the individuals involved with the recreational practice of ballroom dance, where the control of the intensity during the sessions is neglected, are endowed with a more favorable cardiovascular profile when compared to sedentary or insufficiently active individuals. Objectives: To evaluate heart rate (HR) and its variability (HRV) in ballroom dance practitioners (PD) compared to sedentary or insufficiently active (CT). Methods: 50 male volunteers $(C T=25 ; \mathrm{PD}=25)$ (Age: $\mathrm{CT}=25.9$ $\pm 4.5 ; \mathrm{PD}=26.6 \pm 6.1$ years; $\mathrm{BMI}: \mathrm{CT}=24.3 \pm 2.4 ; \mathrm{PD}=24.3 \pm 2.2 \mathrm{~kg} / \mathrm{m}^{2}$ ) were subjected to analysis of heart rate (HR) and its variability in resting conditions (supine and standing) during a submaximal exercise test and in recovery period post effort. Results: PD group showed lower HR values in the supine $(p=0.001)$ and orthostatic $(p=0.05)$ position, in the initial stage of the exercise stress test $(\mathrm{p}=0.003)$, increased exercise tolerance as measured by test time $(p=0.0001)$ and increased heart rate decrement on the first $(p=0.007)$, second $(p=$ $0005)$, third $(\mathrm{p}=0.0001)$, fourth $(\mathrm{p}=0.005)$ and fifth $(\mathrm{p}=0,01)$ minute of recovery. The analysis of heart rate variability at rest, although part of the indices have favored the PD group, the findings were inconsistent. However, the HRV threshold was observed in larger effort intensities in PD group $(\mathrm{p}=0.0001)$, which also showed a greater degree of autonomic modulation in the recovery period. Conclusion: The ballroom dance practitioners evaluated in the present study had a slower chronotropism compared to the control group in all evaluated functional conditions. Additionally, we have observed strong evidence that this group is also contemplated with a higher degree of cardiac autonomic modulation in rest conditions, stress and recovery after stress test compared to the control group.

Key words: autonomic nervous system, heart rate variability, exercise and ballroom dance 


\section{1-Introdução}

Importantes estudos revelam que limitados níveis de aptidão cardiorrespiratória estão associados ao surgimento de doenças crônicas não transmissíveis e mortalidade por todas as causas (CARNETHON et al., 2005; LEE et al., 2010; FARRELL et al., 2013; KHAN et al., 2014; SAWADA et al., 2014). Por outro lado, um crescente corpo de evidências demonstra que a prática regular de exercícios físicos surge como uma estratégia não farmacológica para o aprimoramento da saúde cardiovascular em diversas populações (SLOAN et al., 2009; HAYKOWSKY et al., 2013; CHRYSOHOOU et al., 2015; GIELEN et al., 2015; MOTAHARI-TABARI et al., 2015).

Dentre as variáveis cardiovasculares que podem ser influenciadas positivamente pela prática de exercícios físicos, a função autonômica cardíaca (FAC), modulada principalmente pelos ramos simpático e parassimpático, tem ganhado bastante atenção na literatura diante da relevância clínica e funcional da manutenção de sua integridade (CARNETHON et al., 2002; THOMAS, 2011; METZLER et al., 2013; HABEK et al., 2014; HARRIS et al., 2014).

Evidências sugerem que uma baixa modulação parassimpática e/ou episódios sustentados de hiperatividade simpática podem afetar negativamente a estabilidade elétrica do coração, tornando este órgão mais suscetível a arritmias letais (BILLMAN, 2002; BILLMAN, 2009). Do ponto de vista funcional, uma baixa capacidade de ajuste autonômico diante de uma condição de estresse (ex: exercício físico) pode tornar o débito cardíaco inadequado à nova condição e induzir episódios de vertigem ou síncope, sintomas evidenciados, por exemplo, em casos de intolerância ao ortostatismo (METZLER et al., 2013).

Nesse contexto, uma série de estudos demonstra que a intervenção por meio de exercícios físicos, especialmente de caráter aeróbio, pode promover alterações autonômicas favoráveis na função cardiovascular, como o aumento no grau de modulação global (simpático e parassimpático), redução de estados sustentados de hiperatividade simpática e aumento na modulação parassimpática sobre o coração (PAGKALOS et al., 2008; KANALEY et al., 2009; SLOAN et al., 2009; GOULOPOULOU et al., 2010; SRIDHAR et al., 2010; DANILOWICZ-SZYMANOWICZ et al., 2013; NEGRAO et al., 2015). Do ponto de vista da regulação cardiovascular, o treinamento físico pode induzir aumentos na sensibilidade do barorreflexo, sistema responsável pelo controle momento a momento da 
pressão arterial (IELLAMO et al., 2000; LOIMAALA et al., 2003; GOULOPOULOU et al., 2010; DANILOWICZ-SZYMANOWICZ et al., 2013).

Entretanto, embora os benefícios da prática regular de exercícios físicos sobre o sistema cardiovascular estejam bem estabelecidos na literatura, dados publicados em periódico de grande relevância científica demonstram que o sedentarismo ainda é um relevante problema no cenário mundial (LEE et al., 2012). No Brasil, foi estimado que a inatividade física seja responsável por $8,2 \%$ dos casos de doença coronariana, $10,1 \%$ dos casos de diabetes do tipo II, 13,4\% dos casos de câncer de mama, 14,6\% dos casos de câncer de cólon e 14,6\% da mortalidade por todas as causas (LEE et al., 2012).

Neste cenário, a dança de salão (DS), devido à suas características motivacionais e recreacionais, surge como uma atraente estratégia na árdua tarefa de combate ao sedentarismo. Quando comparada à programas de exercícios convencionais, a dança pode promover benefícios funcionais e cardiovasculares similares, entretanto, com maior nível motivacional por parte dos praticantes (KALTSATOU et al., 2013). Assim, a DS tem sido incorporada em programas de reabilitação de diferentes condições clínicas, contribuindo positivamente para aderência ao programa de exercícios e consequentemente para o sucesso das intervenções (BELARDINELLI et al., 2008; HACKNEY e EARHART, 2009; KALTSATOU et al., 2013).

Por outro lado, embora estudos demonstrem a importância da dança de salão no contexto clínico, no âmbito recreacional, onde o aprendizado dos gestos motores é o principal objetivo, o controle da intensidade durante as sessões de dança é usualmente negligenciado. Dessa forma, ainda é questionado na literatura se a prática recreacional da dança pode gerar sobrecarga fisiológica suficiente para promover adaptações cardiovasculares positivas nos praticantes da modalidade (WYON et al., 2004; DOMENE e EASTON, 2014; DOMENE et al., 2014).

Embora alguns estudos tenham sido conduzidos com objetivo de responder a esta questão, os dados disponíveis na literatura ainda são limitados e controversos (COHEN et al., 1982; WYON et al., 2004; DOMENE e EASTON, 2014; DOMENE et al., 2014). Assim, ainda é obscuro se a prática recreacional de DS, em que a intensidade do esforço não é manipulada, pode promover adaptações favoráveis na função cardiovascular. Dessa forma, questiona-se se indivíduos envolvidos nesta modalidade possuem marcadores de saúde cardiovascular mais favoráveis quando comparados a indivíduos sedentários. 
Desta forma, o objetivo primário do presente estudo foi analisar o comportamento cronotrópico e o da variabilidade da frequência cardíaca (VFC) de praticantes de dança de salão comparativamente à indivíduos sedentários ou insuficientemente ativos no repouso, durante o esforço físico e na recuperação após o esforço físico.

Para uma melhor compreensão das análises que serão discutidas ao longo do trabalho, será apresentada na sequência uma visão geral sobre o sistema cardiovascular, bem como a fundamentação teórica da utilização da VFC e do cronotropismo cardíaco como indicadores de saúde nesse sistema.

\section{1-Visão geral do sistema cardiovascular e sua regulação}

O sistema cardiovascular é composto por três estruturas principais, o coração, os vasos sanguíneos e o sangue. Esse conjunto de estruturas exerce a nobre função de proporcionar um fluxo sanguíneo adequado aos diferentes tecidos e sistemas com o objetivo de suprir suas necessidades metabólicas individuais, garantindo assim a integridade funcional de todo o organismo (THOMAS, 2011).

Entretanto, diferentes fatores intrínsecos e extrínsecos podem levar ao aumento ou redução da necessidade metabólica desses diferentes tecidos, sendo de fundamental importância a existência de um sistema de "gerenciamento" da distribuição do fluxo sanguíneo. Por exemplo, logo após a mudança postural, como exemplo, da posição supina para posição ortostática, parte do fluxo é direcionado aos membros inferiores devido à força da gravidade, e na inexistência de um sistema de ajuste, o retorno venoso seria prejudicado, o que levaria ao desencadeamento de uma síncope (METZLER et al., 2013).

Tal "sistema de gerenciamento da função cardiovascular" é mediado por meio do sistema nervoso autônomo (SNA), que tem como principais agentes reguladores os ramos simpático e parassimpático (COOTE, 2010; THOMAS, 2011; AIRES, 2012). Diferente do sistema nervoso somático, o SNA é composto por dois neurônios em série que conectam o sistema nervoso central (SNC) ao órgão efetor, nesse caso, coração e vasos sanguíneos. Após deixar o SNC, o primeiro neurônio faz sinapse com o segundo formando um gânglio, assim, esses são conhecidos como fibras pré e pós-ganglionares, respectivamente (AIRES, 2012). 
No ramo simpático, as fibras pré-ganglionares deixam o SNC entre o primeiro segmento torácico e o terceiro segmento lombar, e os gânglios se encontram em cadeia próximos à medula espinhal. As fibras pré-ganglionares liberam acetilcolina em seus termais axônicos, enquanto as fibras pós-ganglionares liberam principalmente noradrenalina. Esse último neurotransmissor se acopla à seus receptores $(\alpha 1, \alpha 2, \beta 1, \beta 2$ ou $\beta 3)$ no órgão alvo e exercem sua função (AIRES, 2012).

No coração, a maior parte dos receptores adrenérgicos é do subtipo $\beta 1$, assim, o aumento da atividade simpática provoca aumento na frequência cardíaca (cronotropismo positivo), na força de contração do miocárdio (inotropismo positivo), na velocidade de condução do impulso elétrico (dromotropismo positivo) e na velocidade de relaxamento do miocárdio (luzitropismo positivo) . Nos vasos sanguíneos, a resposta é dependente do subtipo de receptor no qual o ligante se acopla, e como na maioria dos vasos os predominantes são $\alpha 1$ e $\alpha 2$, a resposta primária à ação adrenérgica é a vasoconstrição periférica (THOMAS, 2011).

Quanto ao ramo parassimpático, esse deixa o SNC a partir do tronco cerebral e do sacro, estando os gânglios localizados próximos ou sobre o órgão efetor. As fibras pré e pósganglionares liberam em seus terminais axônicos o neurotransmissor acetilcolina, este se acopla aos seus receptores (muscarínicos ou nicotínicos) modulando assim a atividade do tecido alvo (AIRES, 2012).

De forma antagônica à ação adrenérgica, a acetilcolina tem efeito inibitório sobre o coração, com pouco ou nenhum efeito direto sobre os vasos sanguíneos. Especificamente, ao se acoplar aos receptores muscarínicos do subtipo M2, nos nodos sinusal e atrioventricular, a acetilcolina provoca a abertura de canais de potássio o que gera uma hiperpolarização da membrana desses tecidos. Como desfecho, observa-se cronotropismo e dromotropismo negativos, sendo a modulação do luzitropismo e do inotropismo uma prerrogativa quase exclusiva do sistema simpático (THOMAS, 2011).

Diante do exposto acima, podemos entender que os ramos simpático e parassimpático atuam de forma antagônica sobre o coração e vasos sanguíneos, aumentando ou reduzindo o débito cardíaco e a resistência vascular periférica de acordo com a demanda imposta ao sistema. Entretanto, para que tais ajustes cardiovasculares ocorram, é necessário que informações sobre níveis pressóricos, condição metabólica, volume sanguíneo e atividade muscular sejam constantemente enviadas ao centro de controle cardiovascular (CCC), localizado no bulbo. 
Esse controle por feedback é mediado por baroceptores, quimioceptores, metaboceptores, mecanoceptores, e receptores cardiopulmonares, sensores periféricos que convergem informações no CCC, especificamente no núcleo do trato solitário (NTS), e este, por sua vez, modula os ajustes autonômicos sobre o sistema cardiovascular (THOMAS, 2011; AIRES, 2012). Para um entendimento detalhado da atuação dos diferentes aferentes periféricos sobre a função cardiovascular, será apresentada na sequência uma descrição individualizada dessas estruturas sensoriais.

Baroceptores: Os baroceptores são sensores periféricos localizados principalmente no arco da aorta e na bifurcação da carótida, e são responsáveis pelo controle momento a momento da pressão arterial (PA). Quando estimulados pelo aumento da PA, aferências são enviadas por meio do nervo depressor aórtico, no caso dos receptores do arco aórtico, e do nervo sinusal, que parte dos receptores carotídeos, os quais se unem aos nevos vago e glossofaríngeo, respectivamente, integrando essas informações no NTS (NEGRÃO e BARRETTO, 2010).

Como desfecho, o NTS ativa o núcleo dorsomotor do vago e o núcleo ambíguo, aumentando o tônus vagal e reduzindo o cronotropismo cardíaco. Em paralelo, há estimulação do bulbo ventrolateral caudal que, por sua vez, inibe o bulbo ventrolateral rostral, reduzindo o tônus simpático sobre o coração, o que causa queda adicional do cronotropismo e inotropismo cardíacos. Em relação aos vasos sanguíneos, a redução da atividade simpática provoca redução do tônus vascular e, em consequência, da resistência vascular periférica (NEGRÃO e BARRETTO, 2010).

Em oposição, quando ocorre uma redução da PA, os neurônios do NTS deixam de estimular as projeções parassimpáticas dos núcleos dorsomotor do vago e ambíguo. Ao mesmo tempo, deixam de estimular o bulbo ventrolateral caudal, reduzindo seu efeito inibitório sobre o bulbo ventrolateral rostral, o que leva a um aumento na eferência simpática sobre o coração e vasos sanguíneos. A redução da atividade parassimpática e o aumento da modulação simpática provocam aumento no cronotropismo, inotropismo, dromotropismo e luzitropismo cardíacos, além do aumento da resistência vascular periférica (NEGRÃO e BARRETTO, 2010).

Quimioceptores: Os quimioceptores são sensores localizados estrategicamente no bulbo (quimioceptores centrais) e na bifurcação da carótida (quimioceptores periféricos). Os sensores periféricos respondem primariamente à hipóxia, enquanto os sensores centrais 
respondem primariamente à hipercapnia (NEGRÃO e BARRETTO, 2010; THOMAS, 2011; AIRES, 2012).

Quando estimulados pelo aumento na pressão parcial de $\mathrm{Co}_{2}\left(\mathrm{Pco}_{2}\right)$ ou redução na pressão parcial de oxigênio $\left(\mathrm{Po}_{2}\right)$, os quimioceptores enviam aferências ao centro de controle respiratório, localizado no bulbo, e a resposta é o aumento da frequência respiratória e do volume corrente. Secundariamente, fibras aferentes projetam-se para o CCC, aumentando a atividade do BVLr, resultando no aumento da atividade simpática sobre o coração e vasos sanguíneos (NEGRÃO e BARRETTO, 2010; THOMAS, 2011).

Mecanorreceptores: São aferentes periféricos localizados no tecido muscular (muscle pressor reflex) que proporcionam ajustes ventilatórios e cardiovasculares com o objetivo de garantir a oferta adequada de oxigênio e a remoção eficiente de metabólitos produzidos nos músculos em atividade. Esse controle é mediado por fibras aferentes do tipo III (mielinizadas) e tipo IV (não mielinizadas), neurônios aferentes primários que se projetam para o $\mathrm{CCC}$, desencadeando como resposta eferente o aumento da atividade simpática e redução da atividade parassimpática (NEGRÃO e BARRETTO, 2010).

As fibras do tipo III são responsivas principalmente ao estresse mecânico provocado pela contração muscular, assim são chamadas de mecanoceptores. Já as fibras do tipo IV, são estimuladas principalmente por subprodutos do metabolismo, dessa forma, são conhecidas como metaboceptores. Entretanto, é importante mencionar que as fibras do tipo III também podem ser ativadas por metabólitos e, da mesma forma, as fibras do tipo IV podem secundariamente serem ativadas por deformação mecânica (NEGRÃO e BARRETTO, 2010).

Receptores cardiopulmonares: São terminações nervosas que se espalham em forma de rede pelas câmaras cardíacas e artérias/veias pulmonares, cujas aferências projetam-se para o bulbo pelo nervo vago e via medula espinhal. Além de mecanoceptores, os receptores cardiopulmonares também são constituídos por fibras quimiossensíveis, que além de estimuladas por estresse mecânico causado pelo enchimento das câmaras cardíacas, também são sensibilizadas por alterações metabólicas (IRIGOYEN et al., 2001; THOMAS, 2011).

Os receptores cardiopulmonares podem ser divididos em três grupos: Os localizados no endocárdio nas junções das grandes veias com o átrio direito e das veias pulmonares com o átrio esquerdo, cujos aferentes são fibras vagais mielinizadas; receptores difusamente distribuídos em todas as câmaras cardíacas e contemplados com fibras vagais não 
mielinizadas e receptores difusamente distribuídos em todas as câmaras cardíacas que enviam suas aferências à medula espinhal por fibras simpáticas mielinizadas e não mielinizadas (IRIGOYEN et al., 2001; THOMAS, 2011).

O primeiro grupo é estimulado pelo enchimento e contração atriais. A distensão mecânica das junções venoatriais provoca um aumento da frequência cardíaca mediado pelo aumento do tônus simpático sobre o nodo sinusal. Esse aumento na frequência cardíaca permite a manutenção do volume cardíaco diante de aumentos no retorno venoso (IRIGOYEN et al., 2001; THOMAS, 2011).

O segundo grupo de aferentes atuam de forma similar aos baroceptores localizados no arco da aorta e no seio carotídeo. Quando estimulados por estresse mecânico, estes provocam aumento da atividade parassimpática e redução do tônus simpático sobre o coração (IRIGOYEN et al., 2001; THOMAS, 2011).

Por fim, o terceiro grupo de aferentes cardiopulmonares é ativado por estresse mecânico ou por substâncias químicas disponibilizadas no epicárdio. Esses aferentes parecem ter um papel cardioprotetor, visto que são sensibilizados por estímulos químicos (bradicinina, ácidos orgânicos e cloreto de potássio) provenientes de regiões isquêmicas do miocárdio, sendo estes de fundamental importância na sinalização da dor, como em casos de angina (IRIGOYEN et al., 2001; THOMAS, 2011).

\section{2-Avaliação clínica da função autonômica cardíaca}

Conforme apresentado na sessão anterior, o controle autonômico da função cardiovascular é de grande relevância funcional, de forma que o comprometimento desse sistema tem importantes implicações clínicas. Dessa forma, vários métodos foram desenvolvidos para avaliar a FAC, dentre eles, destacam-se o decremento da FC após a realização de um teste de esforço (DFC) e a variabilidade da frequência cardíaca (VFC), métodos práticos, válidos, não invasivos e com alto poder prognóstico para avaliação clínica e funcional do sistema nervoso autônomo cardíaco (IMAI et al., 1994; PIERPONT e VOTH, 2004; VANDERLEI et al., 2009; CHAPLEAU e SABHARWAL, 2011).

Com o objetivo de fundamentar a utilização da VFC e do DFC como indicador da avaliação da FAC será realizada uma descrição metodológica dessas análises, suas interpretações fisiológicas e implicações clínicas. 


\section{Variabilidade da frequência cardíaca}

O constante ajuste autonômico sobre o sistema cardiovascular, especialmente sobre o coração, provoca oscilações temporais entre ciclos cardíacos consecutivos. Este fenômeno, conhecido como VFC, reflete a capacidade de ajuste do sistema cardiovascular e tem sido amplamente utilizado como uma importante ferramenta para avaliação da FAC em diversas condições clínicas e funcionais (GOLDBERGER et al., 2006; BALCIOGLU et al., 2007; BHAGYALAKSHMI et al., 2007; BAUMERT et al., 2009; VANDERLEI et al., 2009; CHAPLEAU e SABHARWAL, 2011; CHEN et al., 2011; GOLDSTEIN et al., 2011; GRANT et al., 2012; DANILOWICZ-SZYMANOWICZ et al., 2013; BOLEA et al., 2014).

A partir do registro dos intervalos R-R (iRR), várias técnicas foram desenvolvidas para avaliar a integridade ou a capacidade de ajuste do sistema nervoso autônomo, dentre elas, destacam-se as análises temporal e espectral da VFC. A análise temporal é realizada principalmente por modelos estatísticos, o que permite a descrição do quanto ocorre de variação ao longo do registro ou da própria dimensão da diferença temporal entre os ciclos cardíacos. Por outro lado, a análise espectral consiste na decomposição do padrão rítmico em diferentes amplitudes de frequências, permitindo a análise dos diferentes padrões observados ao longo do registro dos iRR (TASK FORCE, 1996; VANDERLEI et al., 2009; SHAFFER; MCCRATY; ZERR, 2014).

A análise no domínio do tempo pode ser realizada por uma série de medidas estatísticas com diferentes aplicações e interpretações fisiológicas. Em registros de curto prazo, os marcadores mais comumente utilizados são o desvio padrão dos iRR normais (DP), o coeficiente de variação (CV), o número de intervalos R-R ao longo do registro (NiRR), a raiz quadrada da média do quadrado das diferenças entre iRR normais adjacentes (r-MSSD), a porcentagem de iRR com duração superior à 50 milissegundos (pNN50) e a média da duração dos iRR em um determinado tempo (Média iRR) (TASK FORCE, 1996; SHAFFER et al., 2014).

Em condição de repouso, estudos com bloqueio farmacológico demonstram que a administração de atropina, um antagonista colinérgico, deprime de forma significativa os índices DP, CV, iRR médio, r-MSSD e pNN50, com alterações não significativas diante da administração de propranolol, um antagonista adrenérgico não seletivo (HAYANO et al., 1991; POLANCZYK et al., 1998). Estes achados sugerem que tais índices podem ser utilizados como indicadores da modulação parassimpática sobre o coração, embora o DP, ou SDNN, seja considerado um indicador de modulação global (TASK FORCE, 1996). 
A análise espectral permite a avaliação das variações rítmicas que ocorrem em 4 amplitudes de frequência, o componente de ultra-baixa frequência $(<0,003 \mathrm{~Hz})$, componente de muito baixa frequência $(0,003$ a $0,004 \mathrm{~Hz})$, componente de baixa frequência $(0,004$ a 0,15 $\mathrm{Hz}$ ) e componente de alta frequência $(0,15$ a $0,4 \mathrm{~Hz})$. A partir dessas bandas de frequência mais dois índices podem ser calculados, a potencia total (área sob a curva em uma frequência de até 0,4 Hz) e a razão Baixa frequência/Alta frequência (TASK FORCE, 1996).

Conforme para os índices temporais, a interpretação fisiológica apresentada na sequência será limitada aos índices adotados em nosso estudo.

Componente de baixa frequência $(\mathrm{BF})$ : Embora o componente de baixa frequência seja rotineiramente utilizado como um marcador da modulação simpática, a administração de atropina provoca depressão deste índice na posição supina (POMERANZ et al., 1985; POLANCZYK et al., 1998; BOLEA et al., 2014), sendo este influenciado pelo ramo simpático apenas na condição ortostática (POMERANZ et al., 1985). Estes dados corroboram com outros importantes achados disponíveis na literatura que revelam que esse intervalo de frequência está associado à função barorreflexa (MOAK et al., 2009; GOLDSTEIN et al., 2011).

Componente de alta frequência (AF): A área espectral de alta frequência tem seu significado fisiológico bem estabelecido na literatura. Também conhecida como "respiratory band", devido à sua relação com o ciclo respiratório, essa área reflete exclusivamente a modulação parassimpática sobre o coração (POMERANZ et al., 1985; POLANCZYK et al., 1998).

Razão baixa frequência/ alta frequência (BF/AF): A associação fisiológica entre os ramos do SNA e os componentes espectrais acima destacados demonstra que a utilização da razão BF/AF como indicador do balanço autonômico cardíaco deve ser no mínimo cautelosa. O destaque está baseado no fato de que seus valores podem ser determinados a partir de alterações em ambos os componentes espectrais e que o BF não é um indicador exclusivo da modulação simpática (SHAFFER et al., 2014).

Área total: Visto que a área total abrange todas as amplitudes de frequência, essa análise não permite uma avaliação individualizada dos componentes do sistema nervoso 
autônomo. Assim, este marcador é frequentemente utilizado como um indicador da modulação autonômica global (ELLER, 2007; MOLINA et al., 2013; TAK et al., 2014).

A)

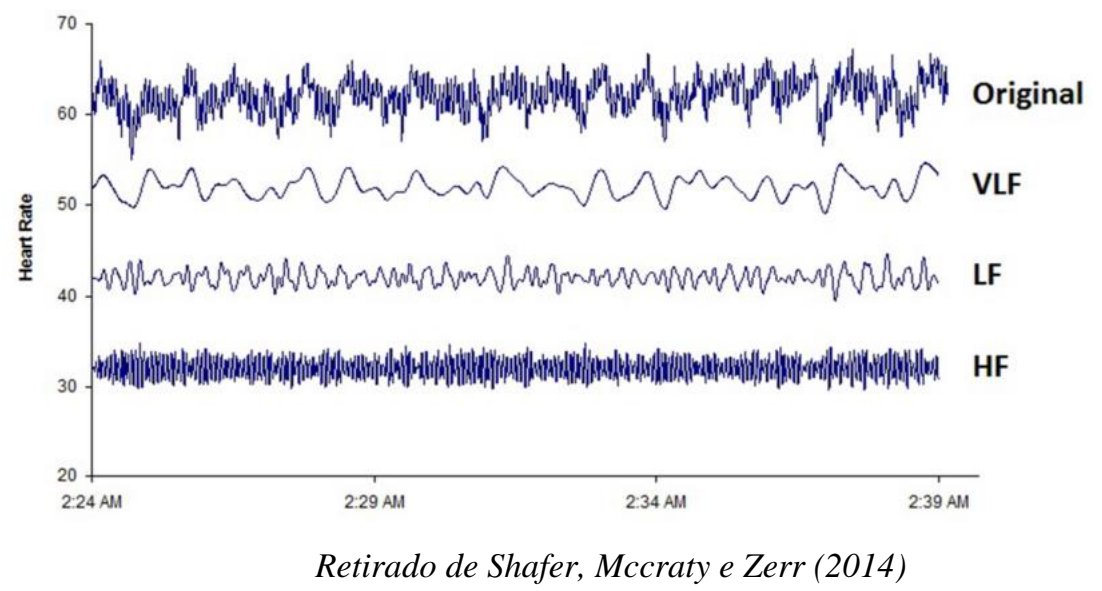

B)

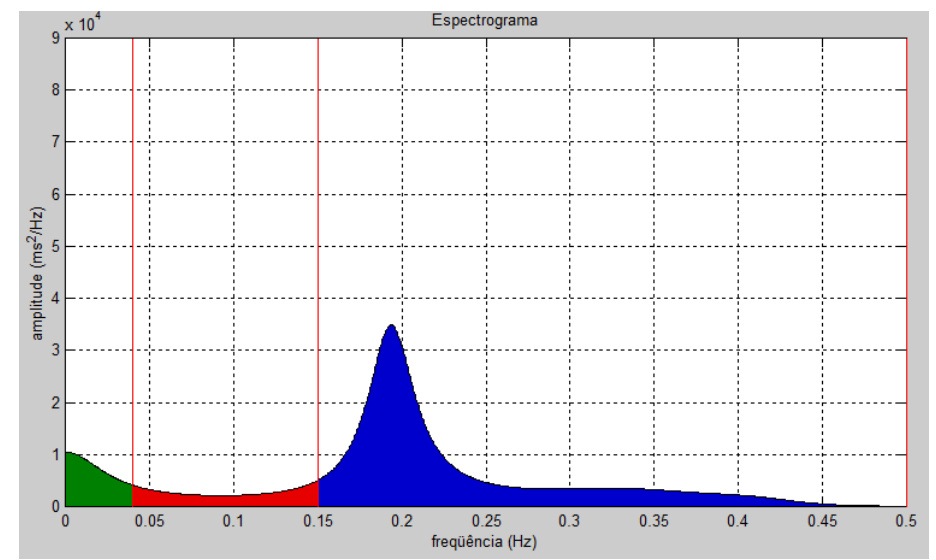

Figura 1: A)-Representação do método de decomposição de ondas (análise espectral).

B)- Dados obtidos a partir do registro de um dos voluntários do presente estudo.

Visto que as análises temporal e espectral são representadas de forma mais adequada em condições estacionárias, a Plotagem de Poincaré e o modelo tempo-frequencial surgem como atraentes técnicas para análise da variabilidade da frequência cardíaca também em condições não estacionárias (TULPPO et al., 1996; MAINARDI et al., 2002).

A plotagem de Poincaré consiste em um método geométrico que representa uma série temporal dentro de um plano cartesiano no qual cada iRR é correlacionado com o intervalo antecedente e definem um ponto no mapa (SMITH et al., 2007). A plotagem de Poincaré permite uma avaliação quantitativa da FAC a partir dos índices SD1, SD2, razão SD1/SD2, área da elipse e coeficiente de correlação.

As variáveis, desvio horizontal (SD2), área da elipse (AE) e coeficiente de correlação (CC) expressam o grau de modulação global da variabilidade da frequência cardíaca. $\mathrm{O}$ 
desvio vertical, SD1, expressa a modulação vagal amostral. A razão entre desvio vertical pelo horizontal (SD1/SD2), expressa o balanço da função autonômica cardíaca durante o período de análise.

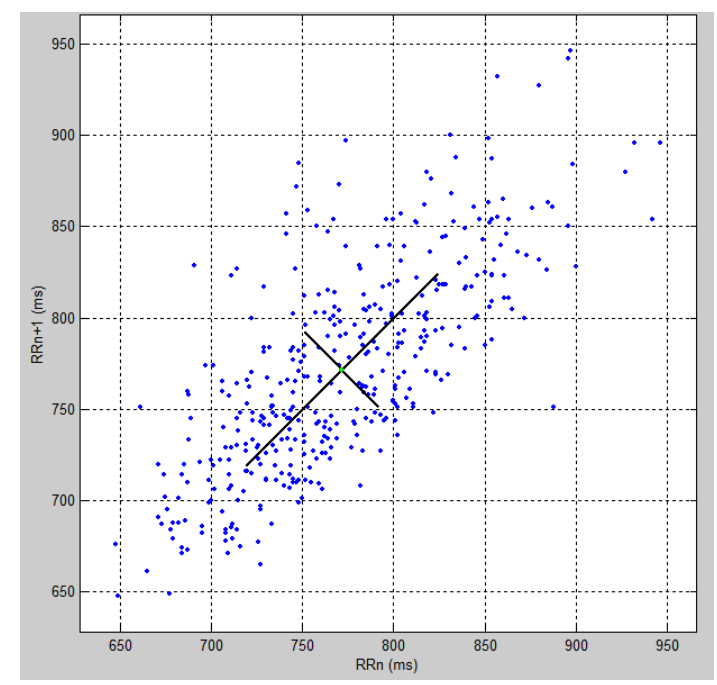

Figura 2- Representação da plotagem de Poincaré. Dados obtidos a partir do registro de um dos voluntários do presente estudo.

No campo da avaliação física, o índice SD1 vem sendo utilizado para identificar a intensidade do esforço físico onde ocorre a depressão ou "platô" da atividade parassimpática, técnica conhecida como "limiar de variabilidade da frequência cardíaca" (LVFC) (LIMA e KISS, 1999; ANOSOV et al., 2000; KARAPETIAN et al., 2008; SALES et al., 2011; GOMES e MOLINA, 2014). Adicionalmente, em diversos estudos observa-se que o ponto de ocorrência do LVFC ligeiramente antecede ou coincide com o limiar anaeróbio, proporcionando valiosas informações quanto ao comportamento neural e metabólico durante a prática de exercícios físicos (LIMA e KISS, 1999; KARAPETIAN et al., 2008; SALES et al., 2011; KARAPETIAN et al., 2012; MOUROT et al., 2012; LEPRÊTRE et al., 2013).

Embora a possível associação fisiológica entre a dinâmica vagal e o limiar anaeróbio ainda não seja conclusiva, algumas tentativas de explicar tal relação estão disponíveis na literatura. Segundo Karapetian, Engels e Gretebeck (2008), o aumento abrupto nas concentrações de lactato sanguíneo pode estar ligado ao aumento expressivo na atividade simpática, fenômeno que ocorreria simultaneamente à depressão vagal. Em adição, este aumento das catecolaminas circulantes poderia resultar em aumento na atividade glicolítica, produção de $\mathrm{CO} 2$ e hiperventilação, fenômenos observados na transição aeróbio-anaeróbio (SCHNEIDER et al., 2000). 


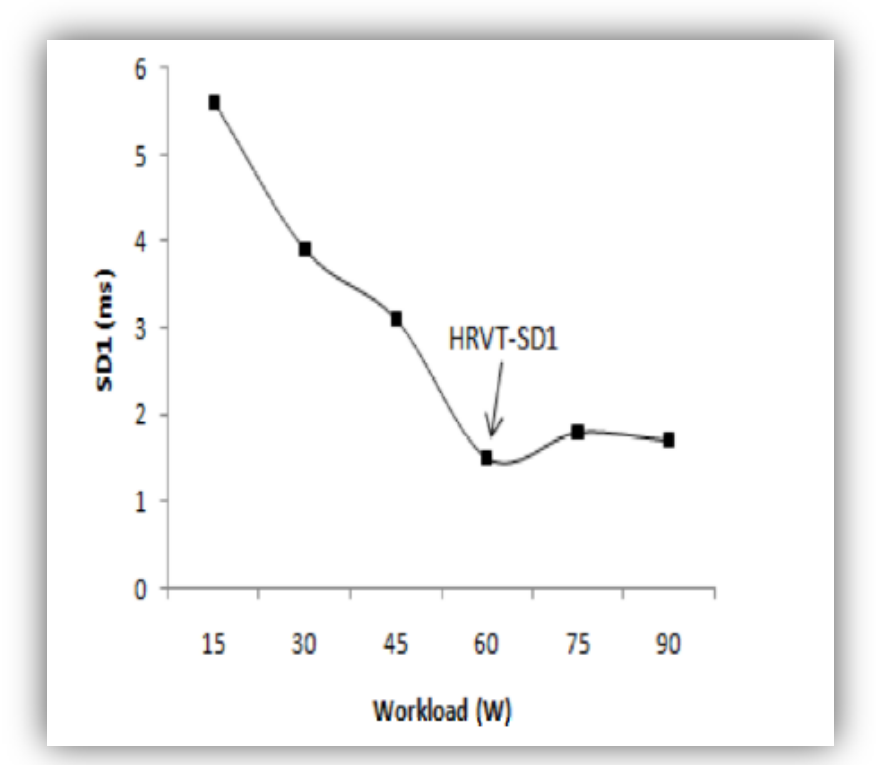

Figura 3: Representação da dinâmica do índice SD1 durante um teste de esforço progressivo. O ponto demarcado com uma seta representa o LVFC (SALES et al., 2011).

Os marcadores do domínio tempo-frequencial utilizados para a análise da variabilidade da frequência cardíaca são apresentados de acordo com os conceitos estabelecidos por Junqueira Jr (conceitos não publicados, 2010). A primeira variável foi chamada de "GRAU", que foi derivada da área espectral total que compreende todo o espectro de frequência, até o limite máximo de 0,50 Hz. A área espectral total (o grau) expressa à magnitude da modulação autonômica global combinada, simpática e parassimpática, de forma dinâmica durante todo período de registro dos intervalos R-R.

A segunda variável foi chamada de "NATUREZA", que é derivada da razão entre as áreas absolutas das faixas de baixa e de alta frequência espectrais, representando assim, o balanço relativo simpato-vagal ao longo de todo o tempo de registro dos intervalos R-R.

A terceira variável foi chamada de "DISTRIBUIÇÃO DA FREQUÊNCIA NO TEMPO”. Essa variável pode ser dividida em duas variáveis distintas chamadas de área > 1 e área $<1$. A variável, área $>1$, expressa o tempo ou período em segundos (s) dos intervalos R$\mathrm{R}$ em que ocorre a simpatoexcitação sobre o coração. Por outro lado, a variável área razão < 1, expressa o tempo ou período em segundos (s) dos intervalos $\mathrm{R}-\mathrm{R}$ em que ocorre a predominância da vagoexcitação sobre o coração. Contudo, destaca-se que foram consideradas as limitações fisiológicas relacionadas à interpretação da razão BF/AF como marcador do balanço autonômico, conforme mencionado anteriormente. 
Ainda no domínio tempo-frequencial utilizou-se da variável Coeficiente de Variação da Razão para expressar a variação global da variabilidade da frequência cardíaca ao longo do período de registro dos intervalos R-R (MOLINA, et al., 2013).

\section{Variabilidade da frequência cardíaca e suas implicações clínicas}

Desde a introdução do termo "homeostase", por Walter Cannon em 1929, os estudos na área da fisiologia foram baseados no princípio de que as células, tecidos e órgão deveriam manter uma condição estática ou constante de "estado estável" em seu ambiente interno. Entretanto, a partir do surgimento de técnicas de processamento de sinais biológicos, observações contínuas do comportamento de fenômenos fisiológicos como a FC, PA e atividade neural, permitiram observar que os processos biológicos sofrem variações complexas e não lineares, mesmo em condições de estado estável (SHAFFER et al., 2014).

Corroborando com essa teoria, diversos estudos foram publicados demonstrando que uma baixa VFC está associada à prognósticos negativos em indivíduos aparentemente saudáveis (JARCZOK et al., 2014; TAK et al., 2014) e em diferentes condições clínicas como doença coronariana (HARRIS et al., 2014), infarto do miocárdio (BOSKOVIC et al., 2014), hipertensão arterial (MELILLO et al., 2012), doença de chagas (JUNQUEIRA JR, 1990; JUNQUEIRA JR, 2012; TRUCCOLO et al., 2013), doença pulmonar obstrutiva crônica (TUKEK et al., 2003), diabetes (BALCIOGLU et al., 2007), entre outras (DE GODOY et al., 2009; RYAN et al., 2011; SUZUKI et al., 2012).

\section{Decremento da frequência cardíaca}

Durante um teste de esforço incremental, ocorre um aumento inicial da frequência cardíaca mediado primariamente pela redução da atividade parassimpática e, posteriormente, pelo aumento progressivo no tônus simpático sobre o coração (ALMEIDA e ARAÚJO, 2003; COOTE, 2010). De forma antagônica, após a interrupção do exercício, a reativação vagal e a desativação simpática são os principais mecanismos envolvidos no decremento da FC (SAVIN et al., 1982; IMAI et al., 1994; ALMEIDA e ARAÚJO, 2003; GOLDBERGER et al., 2006).

Em um elegante estudo com bloqueio farmacológico, Imai et al. (1994) demonstraram que o decremento da FC após um teste de esforço é dependente inicialmente da reativação vagal e posteriormente modulado pela interação entre a reativação vagal e a retirada simpática. Dessa forma, o decremento da FC no primeiro minuto após um teste de esforço é 
rotineiramente interpretado como um indicador da modulação parassimpática, especificamente da capacidade de reativação vagal (COLE et al., 1999; COLE et al., 2000; JAE et al., 2008; JAE et al., 2009; MADDOX et al., 2009; KEYHANI et al., 2013).

Apesar das grandes contribuições dos clássicos estudos com bloqueio farmacológico previamente citados, algumas evidências sugerem que a participação dos diferentes ramos do SNA sobre a redução da FC no primeiro minuto após o estresse físico pode ser modulada pela reativação vagal, desativação simpática ou ambos (KANNANKERIL et al., 2004; BUCHHEIT,LAURSEN, et al., 2007). Em resumo, a reativação vagal parece ser o mecanismo primário para o descenso inicial da FC após a realização de esforço submáximo (IMAI et al., 1994), no entanto, existem indícios de que a desativação simpática seja responsável, ao menos parcialmente, por essa redução após a realização de estímulos de alta intensidade (BUCHHEIT,LAURSEN, et al., 2007; BUCHHEIT,PAPELIER, et al., 2007).

Apesar da discussão acerca da contribuição dos ramos simpático e parassimpático sobre o decremento da frequência cardíaca, a contribuição primária do SNA sobre essa variável é consenso na literatura. Dessa forma, a lenta redução da FC após a realização de um teste de esforço é rotineiramente interpretada como um indicador de disfunção autonômica cardíaca (COLE et al., 1999; COLE et al., 2000; DIMOPOULOS et al., 2006; NAKAMURA et al., 2013; GUERRA et al., 2014).

Decremento da frequência cardíaca e suas implicações clínicas

Um grande conjunto de evidências publicadas nos últimos anos sustenta o potencial valor prognóstico do decremento da frequência cardíaca após o esforço em diversas condições clínicas (COLE et al., 1999; COLE et al., 2000; NISHIME et al., 2000; PANZER et al., 2002; VIVEKANANTHAN et al., 2003; FEI et al., 2005; SMITH et al., 2005; JAE et al., 2008; JAE et al., 2009; MADDOX et al., 2009; ARENA et al., 2010; SAVONEN et al., 2011; YAMADA et al., 2011). Em uma coorte de seis anos, Cole et al. (1999), fizeram um acompanhamento de 2.428 pacientes que foram submetidos a um teste de esforço máximo e verificaram que o decremento da $\mathrm{FC} \leq$ a 12 batimentos por minuto é um poderoso preditor de mortalidade. Pouco depois, observaram que mesmo após um teste de esforço submáximo, e com uma amostra livre de complicações cardiovasculares, o descenso da frequência cardíaca continua sendo um importante fator prognóstico de mortalidade (COLE et al., 2000). 
A partir desses estudos, os pontos de corte de 12 e 42 bpm no primeiro e segundo minuto de recuperação, respectivamente, vêm sendo utilizados na literatura com o objetivo de verificar seus valores prognósticos em diferentes populações (NISHIME et al., 2000; PANZER et al., 2002; VIVEKANANTHAN et al., 2003; SAVONEN et al., 2011; DHOBLE et al., 2014; HA et al., 2014). Independente do ponto de corte estabelecido, a recuperação lenta da FC está associada à prognósticos negativos em indivíduos aparentemente saudáveis (COLE et al., 2000; JAE et al., 2009; DHOBLE et al., 2014), com doença pulmonar obstrutiva crônica (LACASSE et al., 2005), diabetes (NEGISHI et al., 2013), síndrome metabólica (SUNG et al., 2006), doença coronariana (GAYDA et al., 2012) e outras condições clínicas (RAMOS et al., 2012; CAHALIN et al., 2013; ANENI et al., 2014).

\section{3- Objetivos:}

Objetivos principais:

Verificar em homens adultos clinicamente normais:

A hipótese de que praticantes de dança de salão comparativamente a indivíduos sedentários e/ou insuficientemente ativos apresentam melhores respostas cronotrópicas e de variabilidade da frequência cardíaca na condição de repouso supino e ortostático, durante o teste de esforço submáximo e no período de recuperação após o teste de esforço em esteira rolante.

Objetivos secundários:

a)- Testar a hipótese de correlação entre os índices da variabilidade da frequência cardíaca registrados na condição de repouso, nas posições supina e ortostática, com o decremento absoluto e relativo da frequência cardíaca após o teste de esforço submáximo.

b)- Testar a hipótese de correlação entre a variação relativa dos índices da variabilidade da frequência cardíaca na transição da posição supina para posição ortostática com o decremento absoluto e relativo da frequência cardíaca após o teste de esforço submáximo. 


\section{2-Métodos}

Trata-se de um delineamento de corte transversal com amostragem não probabilística por conveniência. A amostra foi composta por 50 voluntários selecionados e distribuídos em 2 grupos: grupo controle $(\mathrm{C} ; \mathrm{n}=25)$, formado por indivíduos sedentários (52\%) ou insuficientemente ativos (48\%) (Tabela 1.1), e grupo de praticantes de dança de salão (PD; n =25), composto por indivíduos que utilizavam apenas a DS como atividade física regular (Tabela 2.2). O nível de atividade física dos voluntários foi classificado de acordo com o Questionário internacional de atividade física-IPAQ (CRAIG et al., 2003).

As modalidades de dança praticadas pelo grupo PD eram o forró, bolero, samba de gafieira, zouk e salsa.

Todas as características individuais dos voluntários estão disponíveis nas Tabelas 1.1 e 1.2. As análises comparativas das variáveis antropométricas e fisiológicas podem ser visualizadas nas Tabelas 1.3 e 1.4 , respectivamente. 
Tabela 1.1: Identificação, ocupação e valores individuais das características dos voluntários inseridos no grupo controle (CT).

\begin{tabular}{|c|c|c|c|c|c|c|}
\hline & Indivíduo & Idade (anos) & Profissão & Peso (kg) & Estatura (cm) & IMC $\left(\mathrm{kg} / \mathrm{m}^{2}\right)$ \\
\hline 1 & ADI & 26 & Jornalista & 59,7 & 161 & 23 \\
\hline 2 & AESC & 30 & Comerciante & 75,9 & 180 & 23,4 \\
\hline 3 & AFJ & 20 & Balconista & 68,8 & 176,6 & 22,1 \\
\hline 4 & BGR & 28 & Agente de viagens & 59,8 & 166,5 & 21,74 \\
\hline 5 & BJJ & 30 & Padeiro & 85 & 174 & 28 \\
\hline 6 & DSL & 23 & Professor & 91,3 & 187,7 & 26,1 \\
\hline 7 & FAZ & 30 & Professor & 83,8 & 176,5 & 26,8 \\
\hline 8 & FDMP & 32 & Comerciante & 67,6 & 164,8 & 24,8 \\
\hline 9 & FHRS & 31 & Técnico de enfermagem & 79,9 & 173,8 & 26,7 \\
\hline 10 & FRR & 25 & Estudante universitário & 86 & 174 & 28 \\
\hline 11 & IAG & 20 & Estudante universitário & 79,5 & 194,7 & 21,4 \\
\hline 12 & JAL & 21 & Estudante universitário & 70,9 & 179,5 & 22 \\
\hline 13 & MRV & 22 & Designer & 61,8 & 173 & 22 \\
\hline 14 & PGR & 34 & Empresário & 62 & 170 & 21,45 \\
\hline 15 & ASS & 28 & Jornalista & 74,2 & 173,3 & 24,7 \\
\hline 16 & $\mathrm{BCCN}$ & 30 & Contador & 80 & 181 & 24,4 \\
\hline 17 & CTS & 20 & Estudante & 75,6 & 172 & 23,3 \\
\hline 18 & EPS & 26 & Técnico em informática & 76,4 & 184,2 & 22,1 \\
\hline 19 & BRR & 31 & Publicitário & 63,9 & 166,5 & 23,1 \\
\hline 20 & RAFR & 26 & Comerciante & 90,1 & 185 & 26,3 \\
\hline 21 & PHFX & 26 & Bancário & 83 & 172 & 28,1 \\
\hline 22 & AHSJ & 20 & Estudante & 63,2 & 172 & 21,4 \\
\hline 23 & PHMS & 20 & Estudante universitário & 87,5 & 180 & 27 \\
\hline 24 & IAP & 20 & Estudante & 66 & 170 & 22,8 \\
\hline 25 & EAP & 29 & Sem ocupação & 101 & 191 & 27,7 \\
\hline
\end{tabular}

$\overline{\mathrm{IMC}}=$ índice de massa corporal 
Tabela 1.2: Identificação, ocupação e valores individuais das características dos voluntários inseridos no grupo de praticantes de dança de salão (PD).

\begin{tabular}{|c|c|c|c|c|c|c|}
\hline & Indivíduo & Idade (anos) & Profissão & Peso (kg) & Estatura (cm) & $\operatorname{IMC}\left(\mathrm{kg} / \mathrm{m}^{2}\right)$ \\
\hline 1 & EASJ & 22 & Estudante universitário & 71,4 & 173,9 & 23,6 \\
\hline 2 & RS & 24 & Web designer & 66,9 & 173,6 & 22,3 \\
\hline 3 & $\mathrm{VM}$ & 20 & Estudante universitário & 77 & 178 & 24,3 \\
\hline 4 & NOE & 22 & Engenheiro & 68,4 & 175,5 & 22 \\
\hline 5 & $\mathrm{ABC}$ & 31 & Comerciante & 79 & 178 & 24,9 \\
\hline 6 & TNB & 23 & Sem ocupação & 72 & 189 & 20,1 \\
\hline 7 & FFS & 38 & Advogado & 62 & 175 & 20,6 \\
\hline 8 & BHSL & 22 & Vendedor & 67,8 & 172,5 & 22,8 \\
\hline 9 & JRBJ & 38 & Vendedor & 65 & 165 & 24,7 \\
\hline 10 & FOL & 28 & Jornalista & 70,9 & 172,2 & 28 \\
\hline 11 & PFV & 22 & Fotógrafo & 80,9 & 182,3 & 24,4 \\
\hline 12 & DRR & 30 & Balconista & 67,1 & 176,4 & 21,6 \\
\hline 13 & JR & 28 & Biólogo & 71,2 & 170 & 24 \\
\hline 14 & WTB & 21 & Professor & 75 & 183 & 22,4 \\
\hline 15 & CGN & 27 & Enfermeiro & 76,2 & 172,5 & 25,6 \\
\hline 16 & NGRM & 25 & Adm. de empresas & 71,9 & 176 & 23,2 \\
\hline 17 & GMLA & 20 & Sem ocupação & 95,2 & 186 & 27,2 \\
\hline 18 & MMAP & 37 & Polícia civil & 89,1 & 182,5 & 26,8 \\
\hline 19 & $\mathrm{MCP}$ & 36 & Engenheiro & 71,1 & 171,4 & 24,2 \\
\hline 20 & TSM & 20 & Estudante universitário & 75 & 174 & 24,8 \\
\hline 21 & DCGS & 26 & Estudante universitário & 84,9 & 169 & 29,7 \\
\hline 22 & LARJ & 26 & Vendedor & 87 & 188 & 24,6 \\
\hline 23 & $\mathrm{CCM}$ & 25 & Taxista & 79 & 182 & 23,8 \\
\hline 24 & DSP & 29 & Auxiliar de serviços gerais & 77 & 169 & 27 \\
\hline 25 & RARJ & 21 & Estudante universitário & 74 & 168 & 26,2 \\
\hline
\end{tabular}

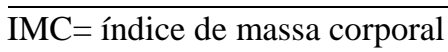


Tabela 1.3: Estatística descritiva amostral da idade e características antropométricas dos grupos controle $(C ; n=25)$ e praticantes de dança de salão (PD; n=25)

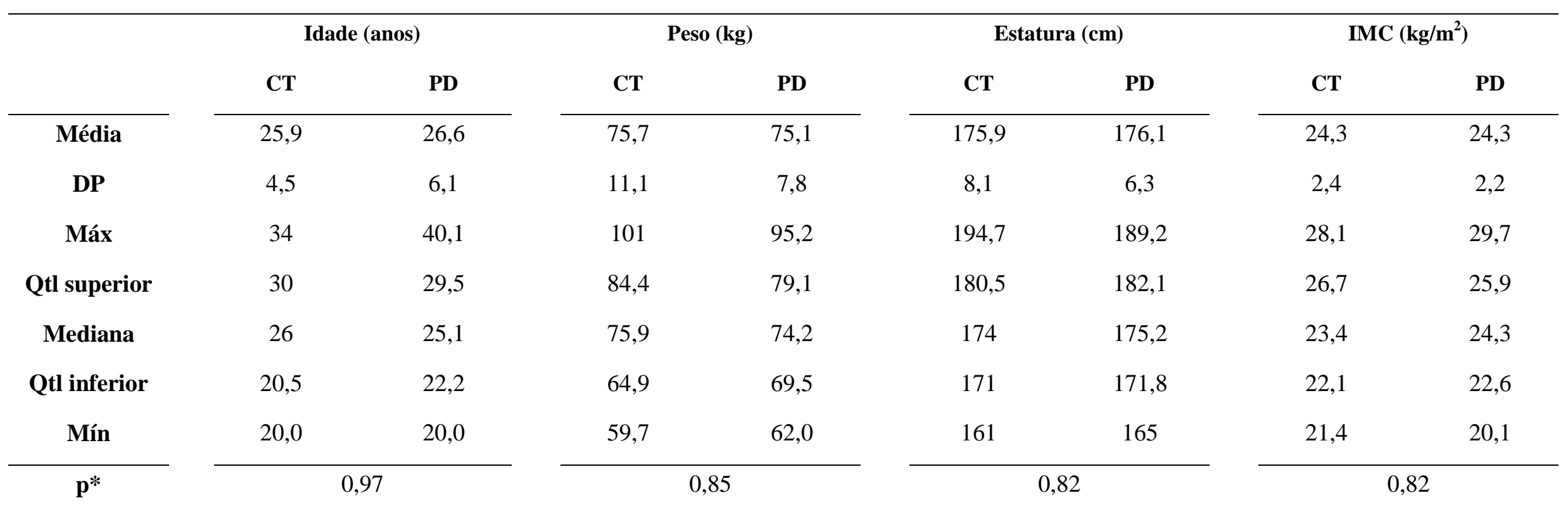

Os grupos foram comparados pelo teste de Mann-Whitney. DP: Desvio padrão; Máx: Valor amostral máximo; Qtl Sup: Quartil superior; Qtl Inf: Quartil inferior; Mín: valor amostral mínimo; CT: Grupo controle; PD: Praticantes de dança de salão 
Tabela 1.4: Estatística descritiva amostral das características fisiológicas dos grupos controle $(C ; n=25)$ e praticantes de dança de salão (PD; $\mathrm{n}=25$ )

\begin{tabular}{|c|c|c|c|c|c|c|c|c|}
\hline & \multicolumn{2}{|c|}{$\mathrm{FC}_{\text {sup }}(\mathrm{bpm})$} & \multicolumn{2}{|c|}{ PAS (mmHg) } & \multicolumn{2}{|c|}{ PAD (mmHg) } & \multicolumn{2}{|c|}{ FR (irpm) } \\
\hline & CT & PD & $\mathbf{C T}$ & PD & CT & PD & CT & PD \\
\hline Média & 70 & 62 & 116 & 115 & 71 & 68 & 15 & 15 \\
\hline DP & 7 & 7 & 8 & 10 & 10 & 8 & 3 & 3 \\
\hline Máx & 85 & 76 & 128 & 138 & 82 & 80 & 20 & 20 \\
\hline Qtl superior & 75 & 70 & 123 & 122 & 80 & 76 & 17 & 18 \\
\hline Mediana & 70 & 62 & 118 & 116 & 76 & 70 & 16 & 14 \\
\hline Qtl inferior & 65 & 55 & 112 & 109 & 64 & 60 & 12 & 12 \\
\hline Mín & 54,0 & 51,0 & 100 & 90,0 & 48 & 52 & 11 & 10 \\
\hline $\mathbf{p}^{*}$ & \multicolumn{2}{|c|}{0,001} & \multicolumn{2}{|c|}{0,62} & \multicolumn{2}{|c|}{0,07} & \multicolumn{2}{|c|}{0,43} \\
\hline
\end{tabular}

Os grupos foram comparados pelo teste de Mann-Whitney. DP: Desvio padrão; Máx: Valor amostral máximo; Qtl Sup: Quartil superior; Qtl Inf: Quartil inferior; Mín: valor amostral mínimo; CT: Grupo controle; PD: Praticantes de dança de salão 


\section{1- Critérios de inclusão/exclusão}

Para proceder à avaliação, os voluntários deveriam apresentar no repouso, previamente ao esforço, valores normais de frequência cardíaca (FC), pressão arterial sistólica (PAS), pressão arterial diastólica (PAD) e índice de massa corpórea (IMC) $>18,5 \mathrm{Kg} / \mathrm{m}^{2}$ e $<29,9$ $\mathrm{Kg} / \mathrm{m}^{2}$. Os voluntários sedentários não deveriam estar envolvidos em atividades físicas regulares à no mínimo 6 meses, sendo exigido o mesmo período mínimo de prática para o grupo PD, não poderiam ser atletas, apresentar doenças cardiovasculares, estar sob tratamento medicamento, possuir qualquer limitação que tornasse inviável a realização do teste de esforço ou apresentar sinais de estado emocional alterado. Adicionalmente, os voluntários foram orientados quanto ao descanso físico de 24 horas previamente ao procedimento experimental e a não consumir bebidas que podem alterar o status autonômico como café, chá, refrigerantes e bebidas energéticas no dia do teste.

\section{2- Procedimentos éticos}

Todos os voluntários, antes do início dos testes, foram orientados quanto aos procedimentos experimentais sendo esclarecidos a respeito dos procedimentos, riscos e benefícios do estudo para posteriormente, em concordância com o estudo, assinarem o termo de consentimento livre esclarecido. O projeto foi inicialmente aprovado sob o parecer $\mathrm{n}^{\circ} 152 \mathrm{~B} / 2011$ e revalidado sob o parecer 791-728/2014 pelo Centro Universitário Euroamericano (UNIEURO).

\section{3- Procedimento experimental}

Todos os testes foram realizados entre 08:00 e 12:00h no Laboratório de Avaliação do Desempenho Físico e da Saúde do Centro Universitário Euro-Americano. Após responderem a uma anamnese compreendendo questões sobre hábitos do cotidiano, uso de medicamentos, histórico de doenças pregressas, etilismo, tabagismo, e ao questionário investigativo sobre o nível de atividade física (IPAQ), os voluntários foram submetidos a medidas antropométricas (massa corporal e estatura), dirigindo-se posteriormente a uma maca onde foram coletadas PA, FC, frequência respiratória (FR) e os iRR na posição supina e posteriormente na posição 
ortostática. Foi realizada também análise eletrocardiográfica de 12 derivações para avaliação clínica cardiológica por parte do professor Dr. Luiz Fernando Junqueira Jr.

Após coletadas as variáveis em repouso, os indivíduos foram submetidos a um teste de esforço submáximo em esteira rolante. O teste era iniciado com uma velocidade de $3 \mathrm{~km} / \mathrm{h}$ e $2 \%$ de inclinação, que eram mantidos por um período de dois minutos. Após o segundo minuto, aumentava-se $1 \mathrm{~km} / \mathrm{h}$ a cada minuto com manutenção da inclinação em $2 \%$ durante todo o protocolo. Ao atingirem $85 \%$ da frequência cardíaca máxima predita por meio da idade (TANAKA et al., 2001), a qual será considerada a frequência cardíaca de pico $\left(\mathrm{FC}_{\text {pico }}\right)$, o protocolo incremental era interrompido e os participantes realizavam o protocolo de recuperação ativa, descrito por Cole et al. (1999), durante cinco minutos na esteira rolante, onde a frequência cardíaca e os intervalos iRR foram continuamente registrados.

Foi utilizado um monitor cardíaco, modelo RS800cx da marca POLAR ${ }^{\circledR}$, para o registro da frequência cardíaca e iRR durante o repouso, esforço e recuperação (PORTO e JUNQUEIRA, 2009). A medida da pressão arterial foi realizada por método auscultatório de acordo com a Sociedade Brasileira de Cardiologia, (2010).

\section{4- Avaliação das variáveis hemodinâmicas}

Após o voluntário assumir a posição supina, e posteriormente a posição ortostática, os dados de FC, FR e PA foram sistematicamente coletados. Toda organização temporal dos registros pode ser visualizada na Figura 4.

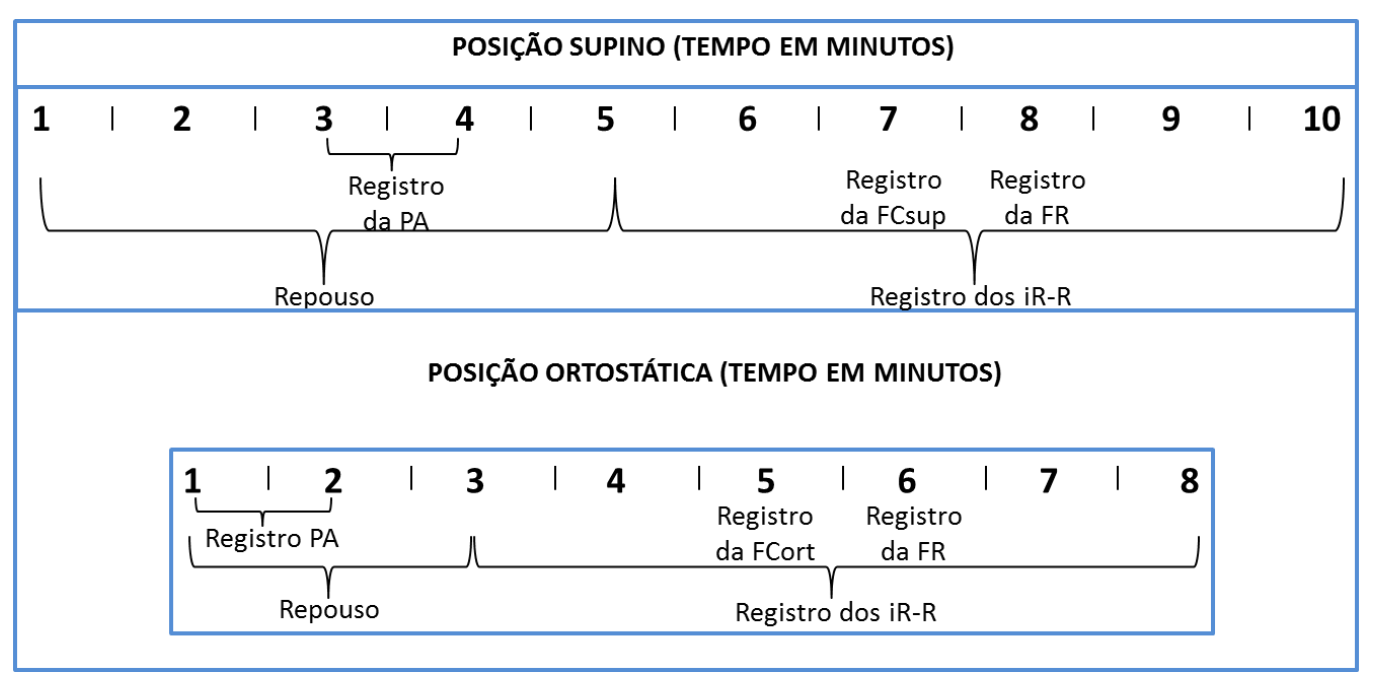

Figura 4- Esquema temporal do registro das variáveis hemodinâmicas. 


\section{5- Avaliação da função autonômica cardíaca}

Para análise da modulação autonômica cardíaca foram utilizados o método da VFC e o decremento da frequência cardíaca após o TES, medidas complementares válidas, práticas, não invasivas e de baixo custo para avaliação clínica e funcional da FAC (POMERANZ et al., 1985; HAYANO et al., 1991; IMAI et al., 1994; POLANCZYK et al., 1998; GOLDBERGER et al., 2006; BUCHHEIT,PAPELIER, et al., 2007).

Diante do não atendimento à alguns pressupostos básicos para análise, quatro indivíduos foram excluídos da avaliação da VFC $(\mathrm{CT} n=2, \mathrm{PD} n=2)$, restando 23 voluntários em cada grupo.

Quadro 1: Voluntários excluídos da análise da VFC

\begin{tabular}{|l|l|}
\hline DSL- Grupo controle & Baixa estacionariedade do sinal \\
\hline FDMP-Grupo controle & Baixa qualidade do registro \\
\hline FOL-Grupo de praticantes de dança & Baixa qualidade do registro \\
\hline WTB- Grupo de praticantes de dança & Frequência respiratória $<10$ ciclos/minuto \\
\hline
\end{tabular}

\section{Variabilidade da frequência cardíaca}

Conforme esquematizado na Figura 1, após cinco minutos em repouso na posição supina, em um ambiente calmo com temperatura controlada $\left(\sim 22^{\circ} \mathrm{C}\right)$, foi iniciado o registro dos iRR com uma duração total de cinco minutos. Em seguida, os mesmos foram orientados a assumirem a posição ortostática, permanecendo nesta condição por mais oito minutos. Após três minutos de ortostatismo, foi realizado mais um registro dos iRR em outra "janela" de 5 minutos. Durante toda a coleta os voluntários foram orientados a evitarem movimentos bruscos e a não conversarem, salvo em algum caso de urgência.

Depois de obtidas as séries temporais de iRR em arquivo texto, os dados passaram por inspeção visual e, posteriormente, foram analisados por meio do software ECGLAB (CARVALHO et al., 2002) desenvolvido no Laboratório Cardiovascular da Faculdade de Medicina em parceria com o Departamento de Engenharia Elétrica da UnB. A análise da VFC foi restrita aos indivíduos com frequência respiratória $\geq 10$ ciclos por minuto, o que evita a sobreposição dos componentes de baixa e alta frequência (BROWN et al., 1993).

Foram selecionados para análise os índices temporais e espectrais da variabilidade da frequência cardíaca, além da plotagem de Poincaré e a análise tempo-frequencial. 
Os índices temporais analisados foram o r-MSSD (raiz quadrada da média do quadrado das diferenças entre intervalos R-R normais adjacentes), DP (desvio padrão de todos os iRR normais), pNN50 (porcentagem dos intervalos R-R com duração superior a 50ms, Média iRR (média de duração dos intervalos R-R em um determinado tempo), Número de iRR (número de intervalos R-R ao longo do registro) e o Coeficiente de variação-CV (indica a variação relativa entorno da média).

Os índices espectrais adotados foram os componentes de baixa frequência $(\mathrm{BF})$, baixa frequência em unidades normalizadas (BFun: BF/AT x 100), alta Frequência (AF), alta frequência em unidades normalizadas (AFun: AF/AT x 100), área total (AT) e a razão BF/AF. A análise espectral foi realizada com base no modelo matemático auto regressivo, com ordem de 16 e taxa de amostragem do sinal de $4 \mathrm{~Hz}$, conforme previamente recomendado (CARVALHO et al., 2003).

No domínio tempo-frequencial foram analisados o GRAU de modulação, a NATUREZA da modulação, a área de modulação com razão maior que 1 e área de modulação menor que 1 .

Do mapa de Poincaré foram extraídos para análise o número de pontos, centróide, área da elipse, SD1, SD2, razão SD1/SD2, e o coeficiente de correlação da plotagem.

Para análise do LVFC, os iRR foram registrados a cada estágio do teste de esforço incremental. Foram adotados dois critérios para identificação desta variável:

1)- primeiro estágio com o valor do índice SD1 < 3,0 ms (LIMA e KISS, 1999; SALES et al., 2011),

2)- dois estágios consecutivos com reduções no índice SD1 < 1,0 ms (BRUNETTO et al., 2005; MOUROT et al., 2012).

Avaliação do desempenho durante o teste de esforço submáximo e o comportamento cronotrópico após o teste de esforço

O desempenho durante o teste de esforço submáximo foi mensurado por meio do tempo, em segundos, despendido para o alcance da $\mathrm{FC}$ alvo $\left(85 \%\right.$ da $\left.\mathrm{FC}_{\max }\right)$. Visto que a redução da frequência cardíaca em condições de esforço submáximo é uma adaptação positiva proporcionada pela prática de exercícios, e que esta é uma medida válida para estimativa do $\mathrm{VO}_{2}$ máximo, torna-se conveniente e prática a sua utilização como medida de desempenho cardiorrespiratório. 
Para avaliação do comportamento autonômico após o TES foram calculadas 3 variáveis, o decremento da frequência cardíaca, o coeficiente de recuperação e a velocidade de decremento da frequência cardíaca.

\section{Decremento da frequência cardíaca}

$\mathrm{O}$ cálculo do decremento da $\mathrm{FC}$ foi realizado subtraindo da $\mathrm{FC}_{\text {pico }}$ os valores de $\mathrm{FC}$ observados no primeiro $\left(\mathrm{DFC}_{1}\right)$, segundo $\left(\mathrm{DFC}_{2}\right)$, terceiro $\left(\mathrm{DFC}_{3}\right)$, quarto $\left(\mathrm{DFC}_{4}\right)$ e quinto minuto $\left(\mathrm{DFC}_{5}\right)$ da fase de recuperação.

\section{Coeficiente de recuperação}

O coeficiente de recuperação foi calculado a partir da razão entre o decremento da frequência cardíaca $\left(1^{\circ}, 2^{\circ} \ldots 5^{\circ}\right.$ minutos de recuperação) e a reserva cronotrópica alcançada durante o teste de esforço. Dessa forma, o coeficiente de recuperação é expresso pela equação: decremento da frequência cardíaca/ $\left(\mathrm{FC}_{\text {pico }}-\mathrm{FC}_{\text {inicial }}\right)$. Este marcador indica o quanto ocorre de recuperação da FC em relação ao seu incremento durante o teste de esforço progressivo e corrige a recuperação da FC em relação aos valores iniciais da FC registrados no início do protocolo de esforço.

\section{Velocidade de decremento da frequência cardíaca}

O cálculo da velocidade de decremento foi realizado a partir da diferença entre os valores de frequência cardíaca registrados entre dois pontos a partir da $\mathrm{FC}_{\text {pico }}$. Por exemplo, se o decremento da frequência cardíaca no segundo minuto foi de $30 \mathrm{bpm}$ e no primeiro o decremento foi de $20 \mathrm{bpm}$, a velocidade de decremento do segundo minuto foi de $10 \mathrm{bpm}$.

\section{6-Procedimentos estatísticos}

Diante do não atendimento ao pressuposto de normalidade de grande parte dos dados, avaliado por meio dos testes de Shapiro-Wilk e Kolmogorov-Smirnov, adotou-se estatística não paramétrica para análise inferencial. 
A estatística descritiva foi apresentada com base nos valores da média, desvio padrão, valor máximo, valor mínimo, mediana e quartis. Tendo em vista que a maioria dos dados apresentou condição não normal, consideramos para efeito de análise e interpretação dos dados a mediana e os quartis.

A comparação entre os grupos no que se refere às diferentes variáveis adotadas no presente estudo foi realizada por meio do teste de Mann-Whitney. Para testar a hipótese de correlação entre as diferentes variáveis adotou-se o teste de correlação de Spearman.

As diferenças entre as diversas comparações instituídas foram consideradas estatisticamente significativas quando as probabilidades bi-caudais das suas ocorrências devidas ao acaso (erro do tipo I) foram menores ou iguais a $5 \%(\mathrm{p} \leq 0,05)$. Considerou-se ainda essas diferenças como tendência estatística ou significância marginal quando $\mathrm{p}>0,05 \mathrm{e}<$ 0,10. O processamento estatístico dos dados foi feito utilizando-se os programas Microsoft Excel e Statistical Package Social Sciences $\quad($ SPSS 20.0) e GraphPad Prism 7 for Windows.

\section{3-Resultados}

Os resultados serão apresentados em três seções. Na primeira, serão destacados os dados cronotrópicos registrados em diferentes condições funcionais e o desempenho durante o TES. Na segunda seção, serão apresentadas as análises da VFC nos domínios temporal, espectral, tempo-frequencial e Poincaré em diferentes condições funcionais, e por fim, as análises de correlação serão destacadas na terceira sessão. 


\section{1- Análise cronotrópica antes, durante e após o teste de esforço submáximo}

Conforme destacado na Tabela 2.1, o grupo PD apresentou menores valores de frequência cardíaca na posição ortostática imediatamente antes do TES $(p=0,05)$, menor frequência cardíaca na fase inicial do teste de esforço $(\mathrm{p}=0,003)$, maior reserva cronotrópica durante o TES $(\mathrm{p}=0,03)$ e um maior tempo de teste $(\mathrm{p}=0,0001)$ quando comparado ao grupo controle.

No período após esforço, o grupo PD apresentou um maior decremento absoluto $(\mathrm{p}=0,001$ a 0,1$)$ e relativo $(\mathrm{p}=0,001$ a 0,006$)$ da frequência cardíaca durante todo o protocolo de recuperação quando comparado ao grupo controle, dados disponíveis nas Tabelas 2.2 e 2.3, respectivamente.

Quanto à velocidade do decremento da frequência cardíaca, a única diferença observada foi no primeiro minuto de recuperação, onde o grupo PD apresentou valores estatisticamente mais expressivos $(\mathrm{p}=0,007)$, dados expressos na Tabela 2.4.

Embora o grupo PD tenha sido contemplado com um maior decremento da frequência cardíaca, a recuperação da frequência cardíaca, avaliada por meio do coeficiente de recuperação, foi similar entre os grupos durante todo o período de recuperação (Tabela 2.5). 
Tabela 2.1: Estatística descritiva amostral dos dados cronotrópicos registrados imediatamente antes e durante o teste de esforço submáximo comparativamente entre os grupos controle $(\mathrm{C} ; \mathrm{n}=25)$ e praticantes de dança de salão (PD; $\mathrm{n}=25)$

\begin{tabular}{|c|c|c|c|c|c|c|c|c|c|c|}
\hline & \multicolumn{2}{|c|}{$\mathrm{FC}_{\text {ort }}(\mathrm{bpm})$} & \multicolumn{2}{|c|}{$\mathrm{FC}_{\text {inicial }}(\mathrm{bpm})$} & \multicolumn{2}{|c|}{$\mathrm{FC}_{\text {pico }}(\mathrm{bpm})$} & \multicolumn{2}{|c|}{ RC (bpm) } & \multicolumn{2}{|c|}{ Tempo de teste (s) } \\
\hline & CT & PD & CT & PD & CT & PD & CT & PD & CT & PD \\
\hline Média & 86 & 81 & 93 & 86 & 164 & 164 & 71 & 76 & 343 & 433 \\
\hline DP & 10 & 11 & 8 & 7 & 4 & 5 & 9 & 7 & 65 & 70 \\
\hline Máx & 108 & 109 & 107 & 105 & 174 & 172 & 96 & 98 & 540 & 610 \\
\hline Qtl superior & 93 & 85 & 98 & 90 & 169 & 169 & 78 & 80 & 375 & 475 \\
\hline Mediana & 88 & 82 & 94 & 86 & 165 & 165 & 70 & 75 & 346 & 420 \\
\hline Qtl inferior & 80 & 74 & 88 & 83 & 161 & 160 & 64 & 71 & 330 & 376 \\
\hline Mín & 80 & 60,0 & 88 & 72,0 & 161 & 153 & 54 & 64 & 300 & 330 \\
\hline $\mathrm{p}^{*}$ & & & & & & & & & & \\
\hline
\end{tabular}

Os grupos foram comparados pelo teste de Mann-Whitney. DP: Desvio padrão; Máx: Valor amostral máximo; Qtl Sup: Quartil superior; Qtl Inf: Quartil inferior; Mín: valor amostral mínimo; CT: Grupo controle; PD: Praticantes de dança de salão; $\mathrm{FC}_{\text {ort: }}$ frequência cardíaca na posição ortostática; $\mathrm{FC}_{\text {inicial: }}$ frequência cardíaca no registrada ao final do período de aquecimento; $\mathrm{FC}_{\text {pico }}$; frequência cardíaca registrada no final do teste de esforço; $\mathrm{RC}$ : reserva cronotrópica $\left(\mathrm{FC}_{\text {pico }}-\mathrm{FC}_{\text {inicial }}\right)$; o tempo de teste consiste no tempo despendido para o alcance da frequência cardíaca alvo (85\% da frequência cardíaca máxima). 
Tabela 2.2: Estatística descritiva amostral do decremento absoluto da frequência cardíaca após o teste de esforço submáximo comparativamente entre os grupos controle $(C ; n=25)$ e praticantes de dança de salão (PD; $n=25)$

\begin{tabular}{|c|c|c|c|c|c|c|c|c|c|c|}
\hline & \multicolumn{2}{|c|}{$\mathrm{DFC}_{1}(\mathrm{bpm})$} & \multicolumn{2}{|c|}{$\mathrm{DFC}_{2}(\mathrm{bpm})$} & \multicolumn{2}{|c|}{$\mathrm{DFC}_{3}(\mathrm{bpm})$} & \multicolumn{2}{|c|}{$\mathrm{DFC}_{4}(\mathrm{bpm})$} & \multicolumn{2}{|c|}{$\mathrm{DFC}_{5}(\mathrm{bpm})$} \\
\hline & CT & PD & CT & PD & CT & PD & CT & PD & CT & PD \\
\hline Média & 33 & 38 & 47 & 53 & 51 & 60 & 55 & 61 & 58 & 64 \\
\hline DP & 8 & 7 & 7 & 6 & 6 & 7 & 6 & 7 & 7 & 7 \\
\hline Máx & 63 & 52 & 60 & 68 & 65 & 74 & 67 & 80 & 72 & 79 \\
\hline Qtl superior & 39 & 44 & 54 & 58 & 56 & 65 & 60 & 66 & 65 & 68 \\
\hline Mediana & 32 & 40 & 45 & 53 & 51 & 58 & 56 & 59 & 59 & 63 \\
\hline Qtl inferior & 28 & 32 & 43 & 50 & 45 & 54 & 48 & 57 & 50 & 59 \\
\hline Mín & 20 & 22 & 29 & 37 & 41 & 48 & 44 & 49 & 47 & 54 \\
\hline $\mathbf{p}^{*}$ & & & & & & & & & & \\
\hline
\end{tabular}

Os grupos foram comparados pelo teste de Mann-Whitney. DP: Desvio padrão; Máx: Valor amostral máximo; Qtl Sup: Quartil superior; Qtl Inf: Quartil inferior; Mín: valor amostral mínimo; CT: Grupo controle; PD: Praticantes de dança de salão; $\mathrm{DFC}_{1}$ a $\mathrm{DFC}_{5}$ : decremento absoluto da frequência cardíaca do primeiro ao quinto minuto 
Tabela 2.3: Estatística descritiva amostral do decremento relativo da frequência cardíaca após o teste de esforço submáximo comparativamente entre os grupos controle $(C ; n=25)$ e praticantes de dança de salão (PD; n=25)

\begin{tabular}{|c|c|c|c|c|c|c|c|c|c|c|}
\hline & \multicolumn{2}{|c|}{$\mathrm{DFC}_{1} \%(\mathrm{bpm})$} & \multicolumn{2}{|c|}{$\mathrm{DFC}_{2} \%(\mathrm{bpm})$} & \multicolumn{2}{|c|}{$\mathrm{DFC}_{3} \%(\mathrm{bpm})$} & \multicolumn{2}{|c|}{$\mathrm{DFC}_{4} \%(\mathrm{bpm})$} & \multicolumn{2}{|c|}{$\mathrm{DFC}_{5} \%(\mathrm{bpm})$} \\
\hline & CT & PD & CT & PD & CT & PD & CT & PD & CT & PD \\
\hline Média & 20,3 & 23,7 & 28,5 & 32,8 & 31,4 & 36,5 & 33,6 & 37,5 & 35,4 & 38,9 \\
\hline DP & 5,3 & 4,8 & 4,5 & 4,2 & 4,0 & 4,2 & 3,6 & 4,4 & 4,5 & 4,0 \\
\hline Máx & 38,4 & 30,4 & 36,5 & 39,8 & 39,6 & 44,8 & 40,2 & 48,5 & 44,1 & 47,9 \\
\hline $\begin{array}{c}\text { Qtl } \\
\text { superior }\end{array}$ & 23,7 & 27,4 & 32,0 & 35,2 & 34,0 & 40,6 & 36,7 & 39,3 & 38,5 & 41,8 \\
\hline Mediana & 19,5 & 23,8 & 27,3 & 32,9 & 30,9 & 36,0 & 33,9 & 36,9 & 35,7 & 38,6 \\
\hline Qtl inferior & 17,2 & 20,6 & 25,7 & 30,1 & 28,2 & 33,6 & 30,4 & 35,2 & 31,2 & 35,8 \\
\hline Mín & 12,5 & 13 & 17,6 & 21,8 & 24,2 & 29,1 & 27,8 & 29,5 & 27,6 & 32,5 \\
\hline $\mathbf{p}^{*}$ & & & & & & & & & & \\
\hline
\end{tabular}

Os grupos foram comparados pelo teste de Mann-Whitney. DP: Desvio padrão; Máx: Valor amostral máximo; Qtl Sup: Quartil superior; Qtl Inf: Quartil inferior; Mín: valor amostral mínimo; CT: Grupo controle; PD: Praticantes de dança de salão; $\mathrm{DFC}_{1} \%$ a $\mathrm{DFC}_{5} \%$ : decremento relativo da frequência cardíaca do primeiro ao quinto minuto 
Tabela 2.4: Estatística descritiva amostral da velocidade do decremento da frequência cardíaca após o teste de esforço submáximo comparativamente entre os grupos controle $(C ; n=25)$ e praticantes de dança de salão (PD; $n=25)$

\begin{tabular}{|c|c|c|c|c|c|c|c|c|c|c|}
\hline & \multicolumn{2}{|c|}{$\mathrm{VD}_{1}$ (bpm) } & \multicolumn{2}{|c|}{$\mathrm{VD}_{2}$ (bpm) } & \multicolumn{2}{|c|}{$\mathrm{VD}_{\mathbf{3}}$ (bpm) } & \multicolumn{2}{|c|}{$\mathrm{VD}_{4}(\mathrm{bpm})$} & \multicolumn{2}{|c|}{$\mathrm{VD}_{5}(\mathrm{bpm})$} \\
\hline & CT & PD & CT & PD & CT & PD & CT & PD & CT & PD \\
\hline Média & 33 & 38 & 13 & 14 & 4 & 6 & 3 & 1 & 3 & 2 \\
\hline DP & 8 & 7 & 5 & 4 & 4 & 4 & 5 & 3 & 4 & 4,0 \\
\hline Máx & 63 & 52 & 23 & 25 & 15 & 15 & 14 & 9 & 14 & 19 \\
\hline Qtl superior & 39 & 44 & 17 & 19 & 7 & 10 & 5 & 4 & 5 & 5 \\
\hline Mediana & 32 & 40 & 15 & 15 & 5 & 5 & 3 & 2 & 2 & 2 \\
\hline Qtl inferior & 28 & 32 & 10 & 10 & 1 & 3 & 0 & -1 & 0 & 0 \\
\hline Mín & 20,0 & 22 & -3 & 8,0 & -6 & -3 & -7 & -9 & -3 & -4 \\
\hline $\mathbf{p}^{*}$ & & & & & & & & & & \\
\hline
\end{tabular}

Os grupos foram comparados pelo teste de Mann-Whitney. DP: Desvio padrão; Máx: Valor amostral máximo; Qtl Sup: Quartil superior; Qtl Inf: Quartil inferior; Mín: valor amostral mínimo; CT: Grupo controle; PD: Praticantes de dança de salão; $\mathrm{VD}_{1}$ a $\mathrm{VD}_{5}$ : velocidade do decremento da frequência cardíaca do primeiro ao quinto minuto; a velocidade do decremento da frequência cardíaca reflete a diferença absoluta entre os valores de frequência cardíaca registrados em dois pontos a partir da $\mathrm{FC}_{\text {pico }}$ 
Tabela 2.5: Estatística descritiva amostral do coeficiente de recuperação da frequência cardíaca após o teste de esforço submáximo comparativamente entre os grupos controle $(C ; n=25)$ e praticantes de dança de salão $(P D ; n=25)$

\begin{tabular}{|c|c|c|c|c|c|c|c|c|c|c|}
\hline & \multicolumn{2}{|c|}{$\mathrm{CR}_{1}$} & \multicolumn{2}{|c|}{$\mathbf{C R}_{2}$} & \multicolumn{2}{|c|}{$\mathbf{C R}_{3}$} & \multicolumn{2}{|c|}{$\mathbf{C R}_{4}$} & \multicolumn{2}{|c|}{$\mathbf{C R}_{5}$} \\
\hline & CT & PD & CT & PD & CT & PD & CT & PD & CT & PD \\
\hline Média & 0,47 & 0,50 & 0,66 & 0,69 & 0,73 & 0,77 & 0,78 & 0,79 & 0,82 & 0,83 \\
\hline DP & 0,12 & 0,10 & 0,11 & 0,08 & 0,08 & 0,08 & 0,08 & 0,09 & 0,08 & 0,07 \\
\hline Máx & 0,88 & 0,66 & 0,85 & 0,85 & 0,90 & 0,98 & 0,91 & 1 & 1 & 1 \\
\hline Qtl superior & 0,53 & 0,57 & 0,75 & 0,73 & 0,81 & 0,84 & 0,84 & 0,85 & 0,89 & 0,87 \\
\hline Mediana & 0,48 & 0,53 & 0,67 & 0,69 & 0,73 & 0,76 & 0,81 & 0,79 & 0,83 & 0,85 \\
\hline Qtl inferior & 0,38 & 0,44 & 0,61 & 0,66 & 0,68 & 0,71 & 0,73 & 0,73 & 0,75 & 0,76 \\
\hline Mín & 0,30 & 0 & 0,41 & 0,5 & 0,53 & 0,61 & 0,61 & 0,61 & 0,63 & 0,7 \\
\hline $\mathrm{p}^{*}$ & \multicolumn{2}{|c|}{0,10} & \multicolumn{2}{|c|}{0,24} & \multicolumn{2}{|c|}{0,10} & \multicolumn{2}{|c|}{0,90} & \multicolumn{2}{|c|}{0,97} \\
\hline
\end{tabular}

Os grupos foram comparados pelo teste de Mann-Whitney. DP: Desvio padrão; Máx: Valor amostral máximo; Qtl Sup: Quartil superior; Qtl Inf: Quartil inferior; Mín: valor amostral mínimo; CT: Grupo controle; PD: Praticantes de dança de salão; $\mathrm{CR}_{1}$ a $\mathrm{CR}_{5}$ : coeficiente de recuperação da frequência cardíaca do primeiro ao quinto minuto; o coeficiente de recuperação foi calculado a partir da razão entre o decremento da frequência cardíaca $\left(1^{\circ}, 2^{\circ} \ldots 5^{\circ}\right.$ minutos de recuperação) e a reserva cronotrópica alcançada durante o teste de esforço. 


\section{2- Análise da variabilidade da frequência cardíaca nas condições de repouso, durante o teste de esforço e no período de recuperação após do teste de esforço submáximo}

\section{Variabilidade da frequência cardíaca na condição de repouso}

$\mathrm{Na}$ análise temporal da VFC, foram observados, na posição supina, maiores valores de Média iRR $(\mathrm{p}=0,05)$ e $\mathrm{r}-\mathrm{MSSD}(\mathrm{p}=0,05)$ no grupo de praticantes de dança em relação ao grupo controle (Tabela 3.1), por outro lado, não foram observadas diferenças entre os grupos na posição ortostática (Tabela 3.2). Da mesma forma, não foram observadas diferenças entre os grupos na variação dos índices temporais na transição da posição supina para posição ortostática (Tabela 3.3).

Quanto à análise espectral, não foram encontradas diferenças significativas entre os grupos na posição supina, dados apresentados na Tabela 4.1. Na posição ortostática, o grupo $\mathrm{PD}$ apresentou maiores valores normalizados de $\mathrm{BF}(\mathrm{p}=0,006)$, menores valores normalizados de $\operatorname{AF}(p=0,006)$ e maior razão $B F / A F(p=0,006)$ quando comparados ao grupo $\mathrm{C}$ (Tabela 4.2). Frente à mudança postural, da posição supina para ortostática, foram observados maiores variações dos índices absoluto $(\mathrm{p}=0,02)$ e normalizado $(\mathrm{p}=0,02)$ de alta frequência, dados expostos na Tabela 4.3 .

No domínio tempo-frequencial, o grupo PD apresentou maiores valores de área total em relação ao grupo controle na posição supina $(\mathrm{p}=0,04)$. Durante o ortostatismo, foram encontrados maiores valores de razão $\mathrm{AF} / \mathrm{BF}(\mathrm{p}=0,01)$ e área $>1(\mathrm{p}=0,01)$, e menores valores de área $<1(\mathrm{p}=0,05)$, dados disponíveis nas tabelas 5.1 e 5.2, respectivamente. Na transição supino-ortostático, foi observada uma maior variação da área $<1$ no grupo $\mathrm{PD}(\mathrm{p}=0,01)$, dados apresentados na Tabela 5.3.

Conforme apresentado na Tabela 6.1, o grupo PD apresentou maiores valores de SD1 $(\mathrm{p}=0,03), \mathrm{SD} 2(\mathrm{p}=0,05)$ e área da elipse $(\mathrm{p}=0,02)$ na posição supina. Por outro lado, não foram observadas diferenças entre os grupos quando avaliados na posição ortostática (Tabela 6.2) ou na variação dos índices diante da alteração postural (Tabela 6.3).

Com o objetivo de apresentar um panorama mais abrangente da análise da variabilidade da frequência cardíaca, foi verificada a proporção de praticantes de dança de salão que apresentaram valores abaixo, dentro ou acima da amplitude interquartil quando utilizado o grupo controle como referência, dados expressos na Tabela 6.4. 
Por fim, com objetivos didáticos, foi elaborada uma tabela que expressa todas as diferenças estatisticamente significativas observadas entre os grupos controle e praticantes de dança de salão (Tabela 6.5). 
Tabela 3.1: Estatística descritiva amostral dos índices temporais da variabilidade da frequência cardíaca durante 5 minutos na posição supina, previamente ao esforço, comparativamente entre os grupos $(\mathrm{CT} ; \mathrm{n}=23)$ e praticantes de dança de salão (PD; $\mathrm{n}=23$ )

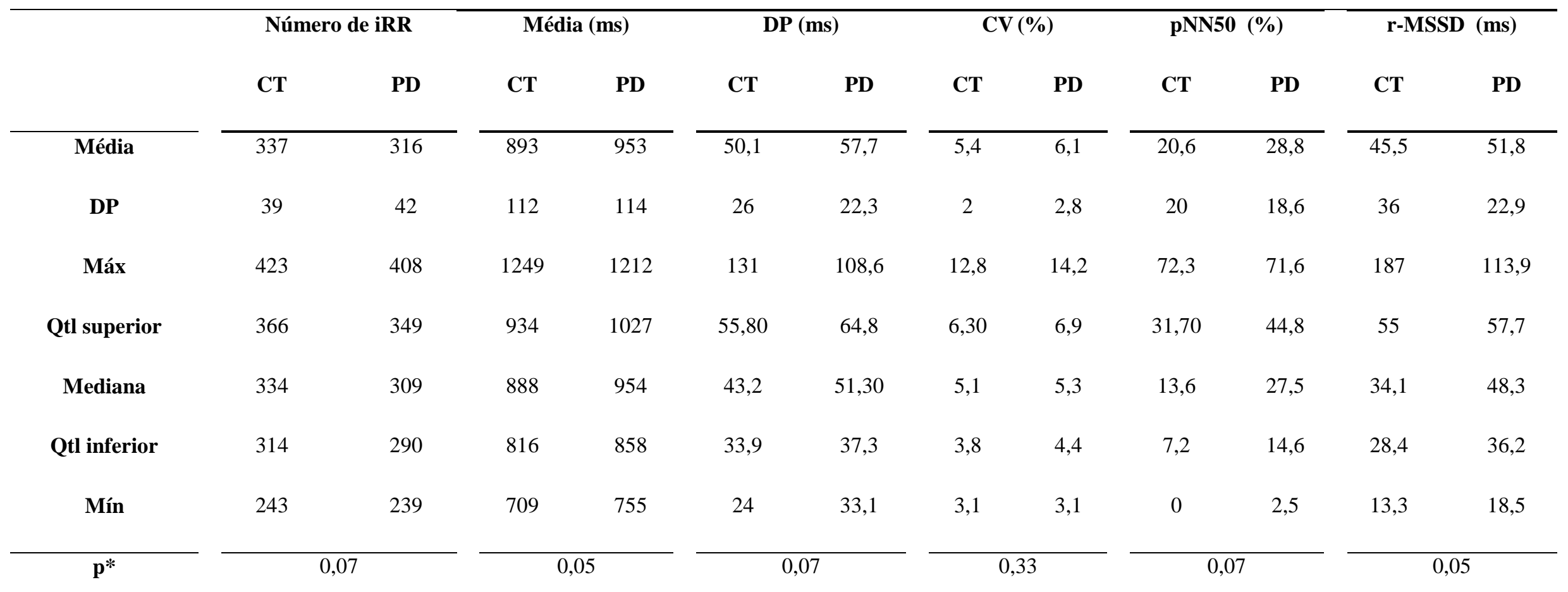

Os grupos foram comparados pelo teste de Mann-Whitney. DP = desvio padrão; CV: coeficiente de variação; Máx : valor amostral máximo; Qtl : quartil; Sup: superior; Inf : inferior; Mín : valor amostral mínimo; CT: grupo controle; PD: praticantes de dança de salão 
Tabela 3.2: Estatística descritiva amostral dos índices temporais da variabilidade da frequência cardíaca durante 5 minutos na posição ortostática, previamente ao esforço, comparativamente entre os grupos controle $(C ; n=23)$ e praticantes de dança de salão $(C ; n=23)$

\begin{tabular}{|c|c|c|c|c|c|c|c|c|c|c|c|c|}
\hline & \multicolumn{2}{|c|}{ Número de iRR } & \multicolumn{2}{|c|}{ Média (ms) } & \multicolumn{2}{|c|}{ DP (ms) } & \multicolumn{2}{|c|}{$\mathrm{CV}(\%)$} & \multicolumn{2}{|c|}{ pNN50 (\%) } & \multicolumn{2}{|c|}{ r-MSSD (ms) } \\
\hline & CT & PD & CT & PD & $\mathbf{C T}$ & PD & CT & PD & CT & PD & CT & PD \\
\hline Média & 422 & 408 & 729 & 733 & 42,9 & 45,1 & 5,8 & 6,1 & 5,3 & 3,6 & 23,7 & 20,9 \\
\hline DP & 70 & 105 & 95 & 97 & 20,7 & 13 & 1,9 & 1,6 & 10,3 & 5,1 & 19,4 & 8,2 \\
\hline Máx & 615 & 668 & 995 & 974 & 125 & 78,0 & 12,5 & 11,5 & 46,7 & 21,5 & 105,3 & 42,3 \\
\hline Qtl superior & 433 & 469 & 782 & 781 & 52 & 53,2 & 6,9 & 6,7 & 4,8 & 5,1 & 25,8 & 25,1 \\
\hline Mediana & 415 & 409 & 723 & 714 & 37,1 & 41,20 & 5,1 & 5,9 & 1 & 1,9 & 18,4 & 19,4 \\
\hline Qtl inferior & 379 & 358 & 664 & 677,00 & 32,3 & 35 & 4,7 & 4,9 & 0 & 0 & 14,9 & 14,2 \\
\hline Mín & 312 & 201 & 577 & 553,0 & 19 & 24,6 & 2,9 & 3,6 & 0 & 0 & 7,6 & 10 \\
\hline p* & & & & & & & & & & & & \\
\hline
\end{tabular}

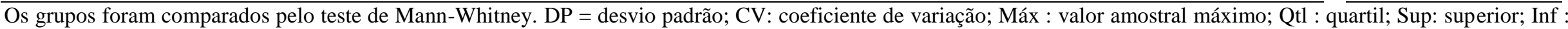
inferior; Mín : valor amostral mínimo; CT: grupo controle; PD: praticantes de dança de salão 
Tabela 3.3: Estatística descritiva amostral da variação relativa dos índices temporais da variabilidade da frequência cardíaca na transição da posição supina para posição ortostática, comparativamente entre os grupos controle $(C ; n=23)$ e praticantes de dança de salão $(C ; n=23)$

\begin{tabular}{|c|c|c|c|c|c|c|c|c|c|c|c|c|}
\hline & \multicolumn{2}{|c|}{$\Delta \%$ Número de iRR } & \multicolumn{2}{|c|}{$\Delta \%$ Média (ms) } & \multicolumn{2}{|c|}{$\Delta \% \mathrm{DP}(\mathrm{ms})$} & \multicolumn{2}{|c|}{$\Delta \% \mathrm{CV}(\%)$} & \multicolumn{2}{|c|}{$\Delta \%$ pNN50 (\%) } & \multicolumn{2}{|c|}{$\Delta \% \mathrm{r}-\mathrm{MSSD}(\mathrm{ms})$} \\
\hline DP & 27,8 & 41,9 & 7,4 & 7,8 & 28,6 & 29,7 & 31,8 & 44,8 & 52,1 & 20,1 & 24,1 & 23,1 \\
\hline Qtl superior & 30,6 & 39,6 & -12 & $-17,1$ & 12,1 & 0 & 38,2 & 25,4 & $-48,6$ & $-68,4$ & $-33,8$ & $-31,2$ \\
\hline Mediana & 19,1 & 28,9 & 19,9 & $-21,1$ & 0 & $-19,6$ & 19,8 & 14,6 & $-87,6$ & $-94,1$ & -44 & $-60,2$ \\
\hline Qtl inferior & 12,2 & 13,3 & $-22,7$ & $-29,3$ & $-29,7$ & $-29,8$ & $-19,3$ & $-11,3$ & $-97,2$ & $-98,6$ & $-60,2$ & $-0,74$ \\
\hline Mín & $-8,1$ & -31 & $-32,7$ & $-41,2$ & $-51,6$ & $-70,5$ & $-36,9$ & $-128,1$ & -100 & -100 & $-78,1$ & $-79,4$ \\
\hline
\end{tabular}

Os grupos foram comparados pelo teste de Mann-Whitney. DP = desvio padrão; CV: coeficiente de variação; Máx : valor amostral máximo; Qtl : quartil; Sup: superior; Inf : inferior; Mín : valor amostral mínimo; CT: grupo controle; PD: praticantes de dança de salão; $\Delta$ diferenças percentuais entre as posições supina e ortostática 
Tabela 4.1: Estatística descritiva amostral dos índices espectrais da variabilidade da frequência cardíaca durante 5 minutos na posição supina, previamente ao esforço, comparativamente entre os grupos controle $(C T ; n=23)$ e praticantes de dança de salão $(C ; n=23)$

\begin{tabular}{|c|c|c|c|c|c|c|c|c|c|c|c|c|}
\hline & \multicolumn{2}{|c|}{ Área Total $\left(\mathrm{ms}^{2}\right)$} & \multicolumn{2}{|c|}{ Área Abs BF $\left(\mathrm{ms}^{2}\right)$} & \multicolumn{2}{|c|}{ Área Nor BF $\left(\mathrm{ms}^{2}\right)$} & \multicolumn{2}{|c|}{ Área Abs AF $\left(\mathrm{ms}^{2}\right)$} & \multicolumn{2}{|c|}{ Área Nor AF $\left(\mathrm{ms}^{2}\right)$} & \multicolumn{2}{|c|}{ Razão BF\AF } \\
\hline & CT & PD & CT & PD & CT & PD & CT & PD & CT & PD & CT & PD \\
\hline Média & 545 & 757 & 128 & 214 & 0,45 & 0,47 & 246 & 264 & 0,54 & 0,51 & 1,1 & 1,1 \\
\hline DP & 662 & 786 & 85 & 187 & 0,18 & 0,18 & 437 & 305 & 0,18 & 0,18 & 1,2 & 0,8 \\
\hline Máx & 2725 & 3368 & 359 & 778 & 0,85 & 0,76 & 2120 & 1248 & 0,87 & 0,90 & 6 & 3,1 \\
\hline Qtl superior & 490 & 641 & 150 & 262 & 0,61 & 0,62 & 205 & 308 & 0,71 & 0,70 & 0,16 & 1,7 \\
\hline Mediana & 302 & 551 & 126 & 158 & 0,42 & 0,53 & 101 & 136 & 0,58 & 0,46 & 0,74 & 1,1 \\
\hline Qtl inferior & 183 & 292 & 57 & 83 & 0,28 & 0,30 & 69 & 81 & 0,39 & 0,37 & 0,40 & 0,43 \\
\hline Mín & 105,0 & 151 & 30 & 24,0 & 0,12 & 0,09 & 18,8 & 42,2 & 0,15 & 0,24 & 0,14 & 0,09 \\
\hline $\mathbf{p}^{*}$ & & & & & & & & & & & & \\
\hline
\end{tabular}

Os grupos foram comparados pelo teste de Mann-Whitney. Abs : absoluta; Nor: área normalizada; BF : baixa frequência; AF : alta frequência; CT; grupo controle; PD: praticantes de dança de salão; DP : Desvio padrão; Máx : Valor amostral máximo; Qtl : Quartil; Sup : superior; Inf : inferior; Mín : valor amostral mínimo 
Tabela 4.2: Estatística descritiva amostral dos índices espectrais da variabilidade da frequência cardíaca durante 5 minutos na posição ortostática, previamente ao esforço, comparativamente entre os grupos controle (CT; $\mathrm{n}=23)$ e praticantes de dança de salão (PD; $\mathrm{n}=23$ )

\begin{tabular}{|c|c|c|c|c|c|c|c|c|c|c|c|c|}
\hline & \multicolumn{2}{|c|}{ Área Total $\left(\mathrm{ms}^{2}\right)$} & \multicolumn{2}{|c|}{ Área Abs BF $\left(\mathrm{ms}^{2}\right)$} & \multicolumn{2}{|c|}{ Área Nor BF $\left(\mathrm{ms}^{2}\right)$} & \multicolumn{2}{|c|}{ Área Abs AF $\left(\mathrm{ms}^{2}\right)$} & \multicolumn{2}{|c|}{ Área Nor AF $\left(\mathrm{ms}^{2}\right)$} & \multicolumn{2}{|c|}{ Razão BF\AF } \\
\hline & CT & PD & CT & PD & CT & PD & CT & PD & CT & PD & $\mathbf{C T}$ & PD \\
\hline Média & 410 & 423 & 169 & 204 & 0,71 & 0,8 & 79 & 44 & 0,27 & 0,19 & 3,2 & 5,8 \\
\hline DP & 504 & 284 & 210 & 166 & 0,10 & 0,11 & 153 & 38 & 0,10 & 0,11 & 2,2 & 3,5 \\
\hline Máx & 2604 & 1375 & 1048 & 724 & 0,91 & 0,93 & 765 & 171 & 0,46 & 0,45 & 10,2 & 13,1 \\
\hline Qtl superior & 445 & 536 & 171 & 239 & 0,79 & 0,89 & 58 & 53 & 0,35 & 0,26 & 3,9 & 8,2 \\
\hline Mediana & 261 & 369 & 107 & 167 & 0,70 & 0,85 & 36 & 35 & 0,30 & 0,15 & 2,3 & 5,7 \\
\hline Qtl inferior & 203 & 209 & 76 & 88 & 0,65 & 0,73 & 28 & 18 & 0,20 & 0,10 & 1,9 & 2,8 \\
\hline Mín & 54 & 124 & 22 & 41,0 & 0,54 & 0,54 & 7,2 & 7,2 & 0,09 & 0,06 & 1,1 & 1,1 \\
\hline p* & & & & & & & & & & & & \\
\hline
\end{tabular}

Os grupos foram comparados pelo teste de Mann-Whitney. Abs : absoluta; Nor: área normalizada; BF : baixa frequência; AF : alta frequência; CT; grupo controle; PD: praticantes de dança de salão; DP : Desvio padrão; Máx : Valor amostral máximo; Qtl : Quartil; Sup : superior; Inf : inferior; Mín : valor amostral mínimo 
Tabela 4.3: Estatística descritiva amostral da variação relativa dos índices espectrais da variabilidade da frequência cardíaca na transição da posição supina para posição ortostática, comparativamente entre os grupos controle (CT; n=23) e praticantes de dança de salão (PD; n=23)

\begin{tabular}{|c|c|c|c|c|c|c|c|c|c|c|c|c|}
\hline & \multicolumn{2}{|c|}{$\Delta$ Área Total $\left(\mathrm{ms}^{2}\right)$} & \multicolumn{2}{|c|}{$\Delta$ Área Abs BF (ms²) } & \multicolumn{2}{|c|}{$\Delta$ Área Nor BF $\left(\mathrm{ms}^{2}\right)$} & \multicolumn{2}{|c|}{$\Delta$ Área Abs AF $\left(\mathrm{ms}^{2}\right)$} & \multicolumn{2}{|c|}{$\Delta$ Área Nor AF $\left(\mathrm{ms}^{2}\right)$} & \multicolumn{2}{|c|}{$\Delta$ Razão BF\AF $(\%)$} \\
\hline & CT & PD & CT & PD & CT & PD & CT & PD & CT & PD & CT & PD \\
\hline Média & $-1,4$ & $-12,3$ & 51,4 & 60,3 & 86,6 & 114,5 & $-54,4$ & $-69,3$ & $-45,6$ & $-58,8$ & 303 & 829 \\
\hline DP & 57,3 & 63,6 & 119 & 177 & 87,2 & 167 & 42,5 & 32,1 & 18,9 & 26,4 & 245 & 1121 \\
\hline Máx & 152 & 165 & 426 & 689 & 375 & 800 & 115 & 30,6 & 4,2 & 4,8 & 828 & 4788 \\
\hline Qtl superior & 34,1 & 7,7 & 87,3 & 72,9 & 146 & 186 & $-45,3$ & $-56,7$ & $-33,3$ & $-37,5$ & 474 & 857 \\
\hline Mediana & $-12,9$ & $-25,4$ & 18,4 & 9,8 & 58,3 & 44,8 & 63,8 & $-83,3$ & $-50,1$ & $-69,7$ & 211 & 441 \\
\hline Qtl inferior & $-48,3$ & $-52,1$ & $-38,5$ & $-39,1$ & 29,7 & 34,3 & $-78,6$ & $-91,6$ & $-60,2$ & $-80,1$ & 94 & 90,3 \\
\hline Mín & $-80,2$ & $-93,2$ & $-71,6$ & $-85,5$ & 0,00 & $-3,5$ & $-89,4$ & $-94,2$ & $-74,3$ & $-88,6$ & 1 & $-7,7$ \\
\hline $\mathbf{p}^{*}$ & \multicolumn{2}{|c|}{0,33} & \multicolumn{2}{|c|}{0,66} & \multicolumn{2}{|c|}{0,71} & \multicolumn{2}{|c|}{0,02} & \multicolumn{2}{|c|}{0,02} & \multicolumn{2}{|c|}{0,13} \\
\hline
\end{tabular}

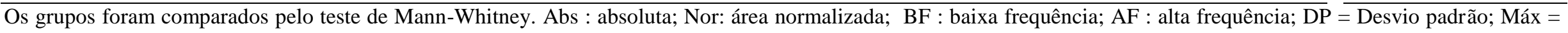
Valor amostral máximo; Qtl = Quartil; Sup = superior; Inf = Quartil inferior; Mín = valor amostral mínimo; CT: grupo controle; PD: grupo de praticantes de dança de salão;

$\Delta$ diferenças percentuais entre as posições supina e ortostática 
Tabela 5.1: Estatística descritiva amostral dos índices tempo-frequenciais da variabilidade da frequência cardíaca durante 5 minutos na posição supina, previamente ao esforço, comparativamente entre os grupos controle (CT; n=23) e praticantes de dança de salão (PD; n=23)

\begin{tabular}{|c|c|c|c|c|c|c|c|c|c|c|}
\hline & \multirow{2}{*}{\multicolumn{2}{|c|}{$\begin{array}{c}\text { Grau } \\
\text { (Área espectral total.ms²) }\end{array}$}} & \multirow{2}{*}{\multicolumn{2}{|c|}{$\begin{array}{c}\text { Natureza } \\
\text { (Razão BF/AF. Mediana) }\end{array}$}} & \multicolumn{4}{|c|}{ Distribuição no tempo } & \multirow{2}{*}{\multicolumn{2}{|c|}{$\begin{array}{l}\text { Variabilidade no tempo } \\
\text { (CV da razão BF/AF. \%) }\end{array}$}} \\
\hline & & & & & \multicolumn{2}{|c|}{ Área > 1 (segundos) } & \multicolumn{2}{|c|}{ Área $<1$ (segundos) } & & \\
\hline & $\mathbf{C T}$ & PD & CT & PD & CT & PD & CT & PD & CT & PD \\
\hline Média & 397 & 599 & 1,1 & 1,2 & 290 & 480 & 75 & 70 & 110 & 113 \\
\hline DP & 470 & 520 & 1,0 & 0,9 & 369 & 830 & 49 & 57 & 40 & 101 \\
\hline Máx & 2396 & 1986 & 4,7 & 3,5 & 1515 & 3737 & 171 & 235 & 216 & 562 \\
\hline Qtl superior & 431 & 752 & 1,5 & 2,0 & 373 & 544 & 124 & 96 & 124 & 106 \\
\hline Mediana & 286 & 449 & 0,9 & 0,8 & 166 & 125 & 59 & 74 & 86 & 95 \\
\hline Qtl inferior & 154 & 241 & 0,5 & 0,6 & 67 & 51 & 36 & 17 & 79 & 82 \\
\hline Mín & 94,3 & 96,7 & 0,2 & 0,1 & 29 & 0 & 3 & 2,5 & 66 & 62 \\
\hline $\mathbf{p}^{*}$ & & & & & & & & & & \\
\hline
\end{tabular}

Os grupos foram comparados pelo teste de Mann-Whitney. DP: Desvio padrão; Máx : Valor amostral máximo; Qtl : Quartil; Sup : superior; Inf : inferior; Mín : valor amostral mínimo; CV : coeficiente de variação 
Tabela 5.2: Estatística descritiva amostral dos índices tempo-frequenciais da variabilidade da frequência cardíaca durante 5 minutos na posição ortostática, previamente ao esforço, comparativamente entre os grupos controle (CT; n=23) e praticantes de dança de salão (PD; n=23)

\begin{tabular}{|c|c|c|c|c|c|c|c|c|c|c|}
\hline & \multirow{2}{*}{\multicolumn{2}{|c|}{$\begin{array}{c}\text { Grau } \\
(\text { Área espectral total.ms²) }\end{array}$}} & \multirow{2}{*}{\multicolumn{2}{|c|}{$\begin{array}{c}\text { Natureza } \\
\text { (Razão BF/AF. Mediana) }\end{array}$}} & \multicolumn{4}{|c|}{ Distribuição no tempo } & \multirow{2}{*}{\multicolumn{2}{|c|}{$\begin{array}{l}\text { Variabilidade no tempo } \\
\text { (CV da razão BF/AF. \%) }\end{array}$}} \\
\hline & & & & & \multicolumn{2}{|c|}{ Área > 1 (segundos) } & \multicolumn{2}{|c|}{ Área $<1$ (segundos) } & & \\
\hline & CT & PD & CT & PD & CT & PD & CT & PD & $\mathbf{C T}$ & PD \\
\hline Média & 353 & 387 & 3,5 & 5,5 & 1215 & 2189 & 16 & 10 & 97 & 98 \\
\hline DP & 471 & 294 & 2,4 & 2,9 & 957 & 1447 & 18 & 20 & 31 & 23 \\
\hline Mediana & 238 & 259 & 2,8 & 4,6 & 924 & 2186 & 6 & 2 & 98 & 95 \\
\hline Qtl inferior & 151 & 198 & 2,0 & 3,6 & 681 & 1046 & 4 & 0 & 79 & 82 \\
\hline Mín & 50 & 128 & 1,1 & 3,6 & 324 & 1046 & 0 & 0 & 58 & 62 \\
\hline
\end{tabular}

Os grupos foram comparados pelo teste de Mann-Whitney. DP: Desvio padrão; Máx : Valor amostral máximo; Qt1 : Quartil; Sup : superior; Inf : inferior; Mín : valor amostral mínimo; CV : coeficiente de variação 
Tabela 5.3: Estatística descritiva amostral da variação relativa dos índices tempo-frequenciais da variabilidade da frequência cardíaca na transição da posição supina para posição ortostática, comparativamente entre os grupos controle (CT; n=23) e praticantes de dança de salão (PD; n=23)

\begin{tabular}{|c|c|c|c|c|c|c|c|c|c|c|}
\hline & \multicolumn{2}{|c|}{$\begin{array}{c}\Delta \% \text { Grau } \\
\left(\text { Área espectral total.ms }{ }^{2}\right)\end{array}$} & \multicolumn{2}{|c|}{$\begin{array}{c}\Delta \% \text { Natureza } \\
\text { (Razão BF/AF. Mediana) }\end{array}$} & \multicolumn{4}{|c|}{$\Delta \%$ Distribuição no tempo } & \multicolumn{2}{|c|}{$\begin{array}{c}\Delta \% \text { Variabilidade no tempo } \\
\text { (CV da razão BF/AF. \%) }\end{array}$} \\
\hline Média & 65 & 74 & 324 & 659 & 798 & 2228 & 69 & 108 & 32 & 30 \\
\hline DP & 132 & 110 & 298 & 749 & 804 & 3886 & 37 & 109 & 32 & 36 \\
\hline Qtl superior & 69 & 85 & 519 & 958 & 916 & 1975 & 92 & 100 & 40 & 34 \\
\hline Mediana & 34 & 53 & 223 & 430 & 526 & 814 & 87 & 95 & 30 & 18 \\
\hline Qtl inferior & 11 & 35 & 86,0 & 100 & 211 & 115 & 59 & 85 & 8 & 4 \\
\hline
\end{tabular}

Os grupos foram comparados pelo teste de Mann-Whitney. DP: Desvio padrão; Máx : Valor amostral máximo; Qtl : Quartil; Sup : superior; Inf : inferior; Mín : valor amostral mínimo; CV : coeficiente de variação; $\Delta$ diferenças percentuais entre as posições supina e ortostática 
Tabela 6.1: Estatística descritiva amostral dos índices do Poincaré da variabilidade da frequência cardíaca durante 5 minutos na posição supina, previamente ao esforço, comparativamente entre os grupos $(\mathrm{CT} ; \mathrm{n}=23)$ e praticantes de dança de salão (PD; $\mathrm{n}=23$ )

\begin{tabular}{|c|c|c|c|c|c|c|c|c|c|c|c|c|c|c|}
\hline & \multicolumn{2}{|c|}{ Número de pontos } & \multicolumn{2}{|c|}{ Centróide (ms) } & \multicolumn{2}{|c|}{ SD1 } & \multicolumn{2}{|c|}{ SD2 } & \multicolumn{2}{|c|}{ Razão SD1/SD2 } & \multicolumn{2}{|c|}{ Área da elipse $\left(\mathrm{ms}^{2}\right)$} & \multicolumn{2}{|c|}{ Coeficiente de correlação } \\
\hline & $\mathbf{C T}$ & PD & CT & PD & CT & PD & $\mathbf{C T}$ & PD & CT & PD & CT & PD & $\mathbf{C T}$ & PD \\
\hline Média & 338 & 322 & 891 & 951 & 31 & 39 & 61 & 74 & 0,49 & 0,54 & 7144 & 10206 & 0,65 & 0,58 \\
\hline DP & 39 & 40 & 105 & 113 & 16 & 17 & 25 & 27 & 0,13 & 0,18 & 7686 & 7934 & 0,14 & 0,24 \\
\hline Máx & 422 & 407 & 1206 & 1212 & 76 & 89 & 152 & 148 & 0,80 & 0,92 & 36377 & 34206 & 0,88 & 0,88 \\
\hline Qtl superior & 366 & 356 & 934 & 1029 & 42 & 45 & 72 & 90 & 0,59 & 0,64 & 7127 & 11236 & 0,74 & 0,77 \\
\hline Mediana & 333 & 318 & 888 & 951 & 26 & 37 & 56 & 68 & 0,49 & 0,54 & 4875 & 8166 & 0,65 & 0,60 \\
\hline Qtl inferior & 313 & 291 & 818 & 858 & 21 & 28 & 43 & 56 & 0,41 & 0,39 & 2785 & 4733 & 0,54 & 0,51 \\
\hline Mín & 249 & 247 & 709 & 754 & 9 & 15 & 33 & 40 & 0,25 & 0,26 & 1027 & 2315 & 0,34 & $-0,20$ \\
\hline $\mathbf{p}^{*}$ & & & & & & & & & & & & & & \\
\hline
\end{tabular}

Os grupos foram comparados pelo teste de Mann-Whitney. DP: Desvio padrão; Máx : Valor amostral máximo; Qtl : Quartil; Sup : superior; Inf : inferior; Mín : valor amostral mínimo; CV : coeficiente de variação 
Tabela 6.2: Estatística descritiva amostral dos índices de Poincaré durante 5 minutos na posição ortostática, previamente ao esforço, comparativamente entre os grupos controle $\quad(\mathrm{CT} ; \mathrm{n}=23)$ e praticantes de dança de salão $(\mathrm{PD} ; \mathrm{n}=23)$

\begin{tabular}{|c|c|c|c|c|c|c|c|c|c|c|c|c|c|c|}
\hline & \multicolumn{2}{|c|}{ Número de pontos } & \multicolumn{2}{|c|}{ Centróide (ms) } & \multicolumn{2}{|c|}{ SD1 } & \multicolumn{2}{|c|}{ SD2 } & \multicolumn{2}{|c|}{ Razão SD1/SD2 } & \multicolumn{2}{|c|}{ Área da elipse $\left(\mathrm{ms}^{2}\right)$} & \multicolumn{2}{|c|}{ Coeficiente de correlação } \\
\hline & CT & PD & CT & PD & CT & PD & CT & PD & CT & PD & CT & PD & CT & PD \\
\hline Média & 440 & 447 & 732 & 734 & 18 & 16 & 61 & 64 & 0,27 & 0,24 & 4714 & 3646 & 0,84 & 0,88 \\
\hline DP & 79 & 81 & 105 & 101 & 15 & 7 & 26 & 21 & 0,08 & 0,06 & 8549 & 2960 & 0,09 & 0,05 \\
\hline Máx & 614 & 667 & 1053 & 980 & 85 & 35 & 160 & 116 & 0,53 & 0,40 & 42977 & 12903 & 0,95 & 0,94 \\
\hline Qtl superior & 479 & 487 & 787 & 781 & 18 & 20 & 72 & 74 & 0,30 & 0,26 & 4842 & 4528 & 0,90 & 0,92 \\
\hline Mediana & 440 & 431 & 723 & 713 & 14 & 14 & 56 & 57 & 0,26 & 0,22 & 2418 & 2697 & 0,87 & 0,90 \\
\hline Qtl inferior & 387 & 414 & 664 & 684 & 11 & 10 & 45 & 50 & 0,22 & 0,19 & 1706 & 1635 & 0,82 & 0,87 \\
\hline Mín & 300 & 300 & 577 & 553 & 5 & 7 & 25 & 37 & 0,15 & 0,17 & 444 & 902 & 0,56 & 0,74 \\
\hline $\mathbf{p}^{*}$ & & & & & & & & & & & & & & \\
\hline
\end{tabular}

Os grupos foram comparados pelo teste de Mann-Whitney. DP: Desvio padrão; Máx : Valor amostral máximo; Qtl : Quartil; Sup : superior; Inf : inferior; Mín : valor amostral mínimo; CV : coeficiente de variação 
Tabela 6.3: Estatística descritiva amostral da variação relativa dos índices de Poincaré na transição da posição supina para posição ortostática, comparativamente entre os grupos controle (CT; $n=23$ ) e praticantes de dança de salão (PD; $n=23$ )

\begin{tabular}{|c|c|c|c|c|c|c|c|c|c|c|c|c|c|c|}
\hline & \multicolumn{2}{|c|}{$\Delta \%$ Número de pontos } & \multicolumn{2}{|c|}{$\Delta \%$ Centróide (ms) } & \multicolumn{2}{|c|}{$\Delta \% \mathrm{SD1}$} & \multicolumn{2}{|c|}{$\Delta \% \mathrm{SD2}$} & \multicolumn{2}{|c|}{$\Delta \%$ Razão SD1/SD2 } & \multicolumn{2}{|c|}{$\Delta \%$ Área da elipse $\left(\mathrm{ms}^{2}\right)$} & \multicolumn{2}{|c|}{$\Delta \%$ Coeficiente de correlação } \\
\hline & $\mathbf{C T}$ & PD & CT & PD & CT & PD & $\mathbf{C T}$ & PD & CT & PD & CT & PD & CT & PD \\
\hline Média & 32 & 42 & 17 & 22 & 46 & 55 & 30 & 27 & 43 & 51 & 58 & 55 & 39 & 84 \\
\hline DP & 32 & 32 & 7 & 8 & 22 & 22 & 24 & 25 & 15 & 15 & 64 & 65 & 36 & 36 \\
\hline Máx & 113 & 170 & 32 & 41 & 94 & 80 & 120 & 90 & 72 & 81 & 328 & 93 & 161 & 545 \\
\hline Qtl superior & 43 & 51 & 22 & 28 & 65 & 73 & 36 & 30 & 51 & 67 & 78 & 79 & 53 & 78 \\
\hline Mediana & 25 & 34 & 19 & 21 & 46 & 63 & 25 & 20 & 48 & 53 & 38 & 68 & 33 & 48 \\
\hline Qtl inferior & 13 & 21 & 11 & 17 & 36 & 35 & 17 & 11 & 32 & 35 & 21 & 30 & 15 & 16 \\
\hline Mín & -8 & -26 & 0,3 & 8 & 5 & 9 & 0,8 & -1 & 11 & 19 & 2,6 & -4 & 6,3 & 1 \\
\hline $\mathbf{p}^{*}$ & \multicolumn{2}{|c|}{0,15} & \multicolumn{2}{|c|}{0,08} & \multicolumn{2}{|c|}{0,17} & \multicolumn{2}{|c|}{0,26} & \multicolumn{2}{|c|}{0,14} & \multicolumn{2}{|c|}{0,45} & \multicolumn{2}{|c|}{0,25} \\
\hline
\end{tabular}

Os grupos foram comparados pelo teste de Mann-Whitney. DP: Desvio padrão; Máx : Valor amostral máximo; Qtl : Quartil; Sup : superior; Inf : inferior; Mín : valor amostral mínimo; CV : coeficiente de variação; $\Delta$ diferenças percentuais entre as posições supina e ortostática 
Tabela 6.4: Proporção de praticantes de dança que apresentaram índices de variabilidade da frequência cardíaca dentro, acima ou abaixo da amplitude interquartílica considerando os registros do grupo controle

\begin{tabular}{|c|c|c|c|c|}
\hline & \multicolumn{2}{|c|}{ Posição supina } & \multicolumn{2}{|c|}{ Posição ortostática } \\
\hline & Índices temporais & Índices espectrais & Índices temporais & Índices espectrais \\
\hline Todos os índices dentro da amplitude interquartil & $8(34 \%)$ & $3(13 \%)$ & $6(26 \%)$ & $2(8 \%)$ \\
\hline Todos os índices abaixo abaixo do $1^{\circ}$ quartil & 0 & 0 & $1(4 \%)$ & 0 \\
\hline Um ou mais índices abaixo do $1^{\circ}$ quartil & $4(17 \%)$ & $9(39 \%)$ & $9(39 \%)$ & $15(65 \%)$ \\
\hline Todos os índices acima do $3^{\circ}$ quartil & $3(13 \%)$ & $1(4 \%)$ & $3(13 \%)$ & 0 \\
\hline Um ou mais índices acima do $3^{\circ}$ quartil & $12(52 \%)$ & $18(78 \%)$ & $11(47 \%)$ & $10(43 \%)$ \\
\hline $\begin{array}{l}\text { Mais de um índice simultaneamente abaixo do } 1^{\circ} \\
\text { ou acima do } 3^{\circ} \text { quartil }\end{array}$ & $13(56 \%)$ & $10(43 \%)$ & $13(56 \%)$ & $9(39 \%)$ \\
\hline
\end{tabular}


Tabela 6.5: Estatística descritiva amostral de todos os índices que apresentaram diferenças entre os grupos controle $(n=23)$ e praticantes de dança $(n=23)$ nas posições supina, ortostática e na variação com a transição da posição supina para posição ortostática $(\mathrm{n}=46)$

\begin{tabular}{|c|c|c|c|}
\hline Índice & Grupo controle & Praticantes de dança & $\mathbf{p}$ \\
\hline \multicolumn{4}{|c|}{ Posição Supina } \\
\hline Média (ms) & $880(816 ; 934)$ & $954(858 ; 1027)$ & 0,05 \\
\hline r-MSSD (ms) & $34,1(28,4 ; 55)$ & $48,3(36,2 ; 57,7)$ & 0,05 \\
\hline Grau $\left(\mathrm{ms}^{2}\right)$ & $286(154 ; 431)$ & $449(241 ; 752)$ & 0,04 \\
\hline SD1 & $26(21 ; 42)$ & $37(28 ; 45)$ & 0,03 \\
\hline SD2 & $56(43 ; 72)$ & $68(56 ; 90)$ & 0,05 \\
\hline Área da elipse $\left(\mathrm{ms}^{2}\right)$ & $4875(2785 ; 7127)$ & $8166(4733 ; 11236)$ & 0,02 \\
\hline \multicolumn{4}{|c|}{ Posição ortostática } \\
\hline Área nor BF (\%) & $0,70(0,65 ; 0,79)$ & $0,85(0,73 ; 0,89)$ & 0,006 \\
\hline Área nor AF (\%) & $0,30(0,20 ; 0,35)$ & $0,15(0,10 ; 0,26)$ & 0,006 \\
\hline Razão BF/AF & $2,3(1,9 ; 3,9)$ & $5,7(2,8 ; 8,2)$ & 0,006 \\
\hline Natureza & $2,8(2,0 ; 4,5)$ & $4,6(3,6 ; 7,5)$ & 0,001 \\
\hline Área >1 (segundos) & $924(681 ; 1337)$ & $2186(1046 ; 2686)$ & 0,01 \\
\hline Área <1 (segundos) & $6(4 ; 33)$ & $2(0 ; 10)$ & 0,05 \\
\hline \multicolumn{4}{|c|}{ Variação supino-ortostático } \\
\hline$\Delta \%$ Área Abs AF (ms2) & $63(-78 ;-45)$ & $-83(-91 ;-56)$ & 0,02 \\
\hline$\Delta \%$ Área Nor AF (\%) & $-50(-60 ;-33)$ & $-69(-80 ;-37)$ & 0,02 \\
\hline$\Delta \%$ Área $<1$ (segundos) & $87(59 ; 92)$ & $95(85 ; 100)$ & 0,01 \\
\hline
\end{tabular}

Os grupos foram comparados pelo teste de Mann-Whitney. Legenda: Ver tabelas anteriores. 
Variabilidade da frequência cardíaca durante e após o teste de esforço submáximo

Durante o teste de esforço incremental, foi observado que o limiar de variabilidade da frequência cardíaca, avaliado por meio do índice SD1, foi identificado de forma mais tardia no grupo PD em relação ao grupo C [300 $(240 ; 360)$ vs $240(180 ; 255)$ segundos, p=0,0001], dados apresentados no Gráfico 1.

Conforme destacado na Tabela 7.1, quando considerados os índices tempofrequenciais nos 5 minutos de recuperação, foi observado um maior valor de área total no grupo de praticantes de dança $(\mathrm{p}=0,04)$, o qual foi acompanhado de maiores valores dos índices centroide $(p=0,02), \operatorname{SD} 2(p=0,01)$ e área da elipse $(p=0,009)$, além de uma tendência a maiores valores no índice SD1 $(\mathrm{p}=0,06)$ na plotagem de Poincaré (Tabela 7.2).

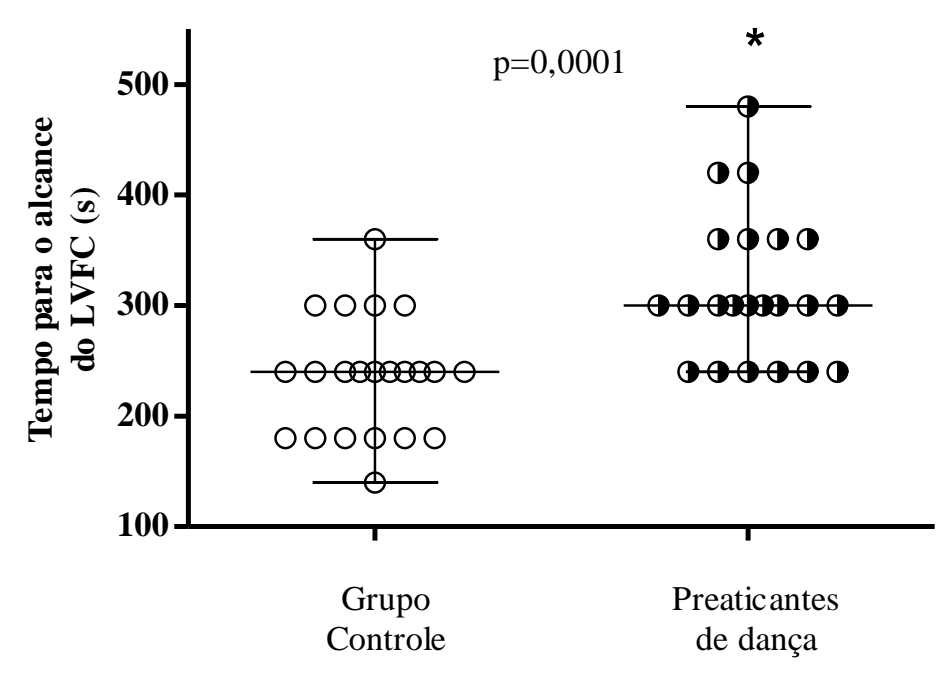

Gráfico 1- Tempo despendido para o alcance do LVFC nos grupos controle e praticantes de dança. Comparação realizada por meio do teste de Mann-Withney. 
Tabela 7.1: Estatística descritiva amostral dos índices tempo-frequenciais da variabilidade da frequência cardíaca registrados no período 5minutos de recuperação após o teste de esforço, comparativamente entre os grupos controle (CT; n=23) e praticantes de dança de salão (PD; n=23)

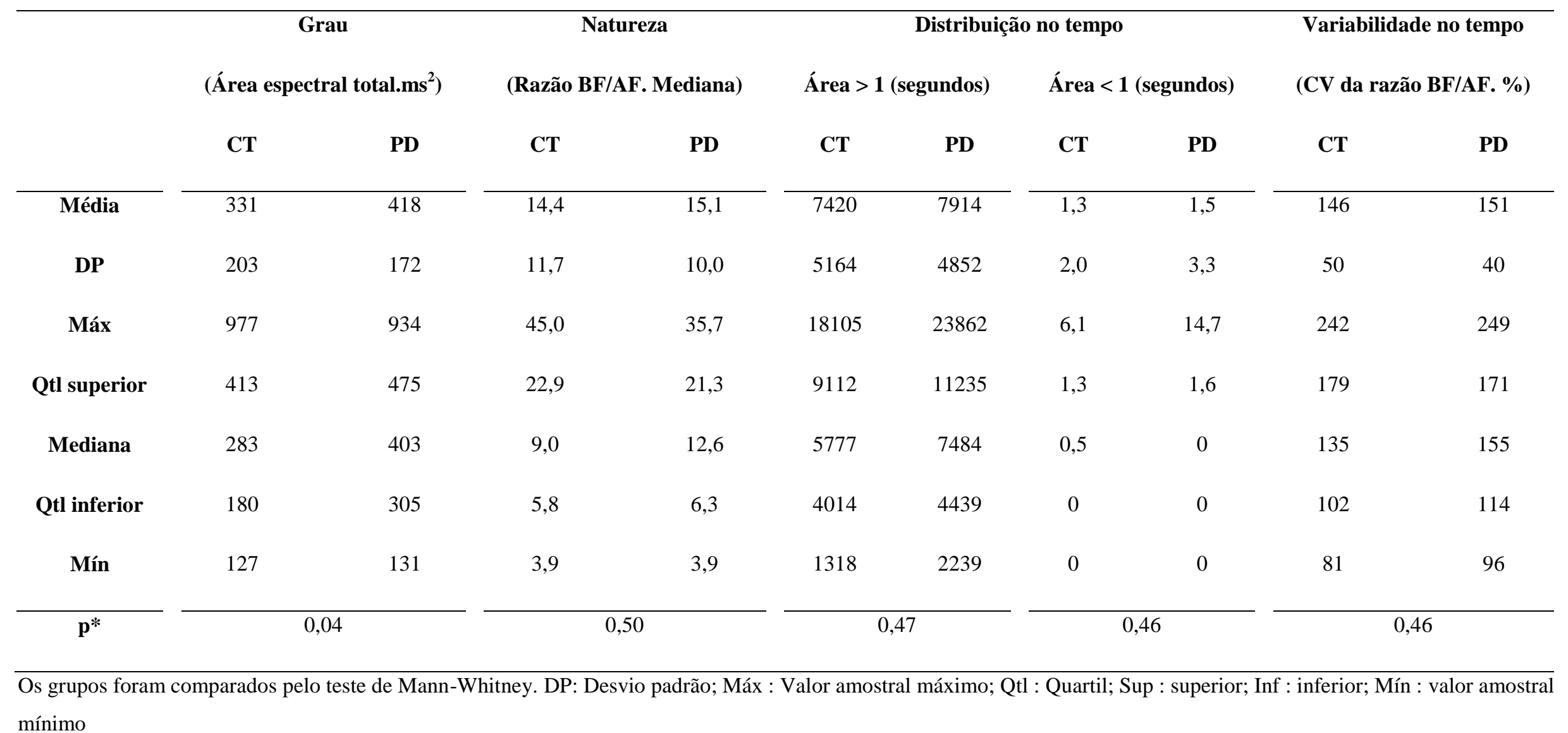


Tabela 7.2: Estatística descritiva amostral dos índices de Poincaré registrados no período de 5minutos de recuperação após o teste de esforço, comparativamente entre os grupos controle (CT; $n=23)$ e praticantes de dança de salão (PD; $n=23)$

\begin{tabular}{|c|c|c|c|c|c|c|c|c|c|c|c|c|c|c|}
\hline & \multicolumn{2}{|c|}{ Número de pontos } & \multicolumn{2}{|c|}{ Centróide (ms) } & \multicolumn{2}{|c|}{ SD1 } & \multicolumn{2}{|c|}{ SD2 } & \multicolumn{2}{|c|}{ Razão SD1/SD2 } & \multicolumn{2}{|c|}{ Área da elipse $\left(\mathrm{ms}^{2}\right)$} & \multicolumn{2}{|c|}{ Coeficiente de correlação } \\
\hline & $\mathbf{C T}$ & PD & $\mathbf{C T}$ & PD & CT & PD & $\mathbf{C T}$ & PD & CT & PD & CT & PD & CT & PD \\
\hline Média & 589 & 584 & 499 & 524 & 4,3 & 5,7 & 87 & 103 & 0,04 & 0,05 & 1278 & 1944 & 0,98 & 0,98 \\
\hline DP & 68 & 47 & 28,9 & 35 & 1,9 & 2,8 & 19 & 69 & 0,01 & 0,02 & 796 & 801 & 0,002 & 0,008 \\
\hline Máx & 656 & 713 & 541 & 579 & 7,5 & 13,8 & 123 & 142 & 0,08 & 0,12 & 2880 & 4856 & 0,99 & 1,00 \\
\hline Qtl superior & 622 & 611 & 516 & 554 & 6,1 & 7,8 & 106 & 116 & 0,06 & 0,06 & 2054 & 2857 & 0,99 & 0,99 \\
\hline Mediana & 600 & 585 & 503 & 517 & 3,9 & 4,8 & 82 & 99 & 0,04 & 0,04 & 871 & 1679 & 0,99 & 0,99 \\
\hline Qtl inferior & 577 & 553 & 476 & 493 & 2,5 & 3,7 & 71 & 88 & 0,03 & 0,03 & 697 & 1016 & 0,99 & 0,99 \\
\hline Mín & 308 & 514 & 423 & 468 & 2,1 & 2,7 & 62 & 69 & 0,02 & 0,02 & 464 & 801 & 0,98 & 0,96 \\
\hline $\mathbf{p}^{*}$ & \multicolumn{2}{|c|}{0,16} & \multicolumn{2}{|c|}{0,02} & \multicolumn{2}{|c|}{0,07} & \multicolumn{2}{|c|}{0,01} & \multicolumn{2}{|c|}{0,44} & \multicolumn{2}{|c|}{0,009} & \multicolumn{2}{|c|}{0,61} \\
\hline
\end{tabular}

Os grupos foram comparados pelo teste de Mann-Whitney. DP: Desvio padrão; Máx : Valor amostral máximo; Qtl : Quartil; Sup : superior; Inf : inferior; Mín : valor amostral mínimo. 


\section{3-Correlação entre a variabilidade da frequência cardíaca e o decremento da frequência cardíaca}

Quando os índices temporais calculados a partir de registros na posição supina foram correlacionados com o DFC, foram encontradas correlações estatisticamente significativas entre a o número de intervalos R-R e o DFC no segundo $\left(r_{s}-0,30 p=0,03\right)$, terceiro $\left(r_{s}-0,35\right.$ $\mathrm{p}=0,01)$, quarto $\left(\mathrm{r}_{\mathrm{s}}-0,44 \mathrm{p}=0,02\right)$ e quinto minuto $\left(\mathrm{r}_{\mathrm{s}}-0,55 \mathrm{p}=0,0001\right)$, entre a média dos i-RR e o DFC no segundo $\left(\mathrm{r}_{\mathrm{s}} 0,38 \mathrm{p}=0,008\right)$, terceiro $\left(\mathrm{r}_{\mathrm{s}} 0,43 \mathrm{p}=0,003\right)$, quarto $\left(\mathrm{r}_{\mathrm{s}} 0,51 \mathrm{p}=\right.$ $0,0001)$ e quinto minuto de recuperação $\left(\mathrm{r}_{\mathrm{s}} 0,62 \mathrm{p}=0,0001\right)$, entre o PNN50 e o DFC do quinto minuto de recuperação $\left(\mathrm{r}_{\mathrm{s}} 0,35 \mathrm{p}=0,01\right)$ e entre o R-MSSD e o DFC no quarto $\left(\mathrm{r}_{\mathrm{s}} 0,28\right.$ $\mathrm{p}=0,05)$ e quinto $\left(\mathrm{r}_{\mathrm{s}} 0,32 \mathrm{p}=0,03\right)$ minuto pós esforço, dados apresentados na Tabela 8.1.

Quanto aos índices temporais calculados a partir de registros na posição ortostática, foram encontradas correlações significativas entre o número de intervalos $\mathrm{R}-\mathrm{R}$ e o decremento da frequência cardíaca no segundo $\left(\mathrm{r}_{\mathrm{s}}-0,28 \mathrm{p}=0,05\right)$, terceiro $\left(\mathrm{r}_{\mathrm{s}}-0,35 \mathrm{p}=0,01\right)$, quarto $\left(\mathrm{r}_{\mathrm{s}}-0,32 \mathrm{p}=0,02\right)$ e quinto minuto $\left(\mathrm{r}_{\mathrm{s}}-0,35 \mathrm{p}=0,01\right)$, entre a média dos $\mathrm{i}-\mathrm{RR}$ e decremento no terceiro $\left(\mathrm{r}_{\mathrm{s}} 0,31 \mathrm{p}=0,01\right)$, quarto (rs $\left.0,38 \mathrm{p}=0,009\right)$ e quinto (rs $\left.0,52 \mathrm{p}=0,001\right)$ minuto e entre o DP e o decremento no quinto minuto de recuperação (rs $0,31 \mathrm{p}=0,03$ ), dados expostos na tabela 8.2.

Quanto à magnitude da variação dos índices temporais na transição da posição supina para posição ortostática, a única correlação estatisticamente significativa encontrada foi entre o $\triangle \mathrm{CV}$ e o DFC no quarto minuto de recuperação (rs -0,39 p=0,007), dados disponíveis na Tabela 8.3.

$\mathrm{Na}$ análise espectral, quando consideradas as análises na posição supina, foram observadas correlações entre Área normalizada BF e o DFC no quinto minuto $\left(\mathrm{r}_{\mathrm{s}} 0,33 \mathrm{p}=\right.$ $0,02)$, entre a área normalizada AF e o DFC no quinto minuto $\left(\mathrm{r}_{\mathrm{s}} 0,33 \mathrm{p}=0,02\right)$ e entre a razão $\mathrm{BF} / \mathrm{AF}$ e o DFC no quinto minuto de recuperação ativa $\left(\mathrm{r}_{\mathrm{s}}-0,33 \mathrm{p}=0,02\right)$ (Tabela 9.1). Na posição ortostática, foi observada correlação significativa apenas entre a área total e o DFC no quinto minuto de recuperação $\left(r_{s} 0,35 \mathrm{p}=0,01\right) \quad$ (Tabela 9.2). Na variação dos índices espectrais diante da manobra postural ativa, foi observada correlação entre a $\Delta$ Área nor BF e o DFC no quinto minuto $\left(\mathrm{r}_{\mathrm{s}}-0,40 \mathrm{p}=0,005\right)$, entre $\Delta$ Área nor AF e o quinto minuto $\left(\mathrm{r}_{\mathrm{s}}-0,28\right.$ $\mathrm{p}=0,05)$ e entre $\Delta$ Razão $\mathrm{BF} / \mathrm{AF}$ e o quarto $\left(\mathrm{r}_{\mathrm{s}}-0,28 \mathrm{p}=0,05\right)$ e quinto minuto de recuperação $\left(r_{\mathrm{s}}-0,35 \mathrm{p}=0,01\right)$, dados apresentados na Tabela 9.3. 
No domínio tempo-frequencial, foram encontradas correlações entre natureza e o DFC no quinto minuto $\left(\mathrm{r}_{\mathrm{s}}-0,31 \mathrm{p}=0,03\right)$, entre a área de razão $>1$ e o DFC no quinto minuto $\left(r_{s}-0,30 p=0,04\right)$ e entre a área de razão $<1$ e o DFC no quinto minuto de recuperação $\left(r_{s} 0,30\right.$ $p=0,04)$. Na posição ortostática, foi observada correlação significativa apenas entre o Grau de modulação e o DFC no quinto minuto após o esforço $\left(r_{s} 0,29\right.$ p= 0,04).

Ainda sobre os índices tempo-frequenciais, quando os mesmos foram analisados diante da alteração postural, foram observadas correlações entre $\Delta$ natureza e o DFC no segundo $\left(\mathrm{r}_{\mathrm{s}} 0,31 \mathrm{p}=0,03\right)$, terceiro $\left(\mathrm{r}_{\mathrm{s}} 0,29 \mathrm{p}=0,04\right)$, quarto $\left(\mathrm{r}_{\mathrm{s}} 0,32 \mathrm{p}=0,02\right)$ e quinto minuto de recuperação $\left(\mathrm{r}_{\mathrm{s}} 0,39 \mathrm{p}=0,006\right)$, entre $\Delta$ Área razão $>1$ e o DFC no segundo $\left(\mathrm{r}_{\mathrm{s}} 0,33 \mathrm{p}=\right.$ $0,02)$, terceiro $\left(\mathrm{r}_{\mathrm{s}} 0,32 \mathrm{p}=0,03\right)$, quarto $\left(\mathrm{r}_{\mathrm{s}} 0,37 \mathrm{p}=0,01\right)$ e quinto $\left(\mathrm{r}_{\mathrm{s}} 0,46 \mathrm{p}=0,01\right)$ minuto da recuperação após o teste de esforço.

Por fim, na posição supina, foram identificadas correlações entre o número de pontos na plotagem de Poincaré e o DFC no terceiro $\left(\mathrm{r}_{\mathrm{s}}-0,33 \mathrm{p}=0,02\right)$, quarto $\left(\mathrm{r}_{\mathrm{s}}-0,41 \mathrm{p}=0,04\right)$ e quinto minuto $\left(\mathrm{r}_{\mathrm{s}}-0,55 \mathrm{p}=0,0001\right)$, e entre o centróide e o DFC no segundo $\left(\mathrm{r}_{\mathrm{s}} 0,38 \mathrm{p}=\right.$ $0,008)$, terceiro $\left(\mathrm{r}_{\mathrm{s}} 0,42 \mathrm{p}=0,03\right)$, quarto $\left(\mathrm{r}_{\mathrm{s}} 0,50 \mathrm{p}=0,0001\right)$ e quinto minuto $\left(\mathrm{r}_{\mathrm{s}} 0,61 \mathrm{p}=\right.$ $0,0001)$.

Na posição ortostática, foram encontradas correlações entre o número de pontos e o DFC no terceiro minuto $\left(\mathrm{r}_{\mathrm{s}}-0,28 \mathrm{p}=0,04\right)$, e entre o centróide e o DFC no terceiro $\left(\mathrm{r}_{\mathrm{s}} 0,35 \mathrm{p}=\right.$ $0,01)$, quarto $\left(\mathrm{r}_{\mathrm{s}} 0,37 \mathrm{p}=0,01\right)$ e quinto minuto $\left(\mathrm{r}_{\mathrm{s}} 0,50 \mathrm{p}=0,0001\right)$. Na variação diante da alteração postural, foram observadas correlações entre $\triangle$ Razão SD1/SD2 e o DFC no segundo minuto $\left(\mathrm{r}_{\mathrm{s}} 0,28 \mathrm{p}=0,05\right)$ e entre o $\Delta$ coeficiente de correlação e o DFC no segundo $\left(\mathrm{r}_{\mathrm{s}} 0,32 \mathrm{p}=\right.$ $0,02)$ e quinto $\left(r_{s} 0,31 p=0,03\right)$ minuto de recuperação após o esforço.

Visto que a análise de correlação entre a variabilidade da frequência cardíaca em repouso e o decremento relativo da frequência produziu resultados da mesma natureza e de magnitude similar quando comparados aos obtidos na análise com o decremento absoluto, os mesmos não serão destacados na forma de texto, contudo, estão disponíveis em tabelas anexadas ao trabalho. 
Tabela 8.1: Correlação $(n=46)$ entre os diversos índices temporais das séries de intervalo RR na posição supina previamente ao esforço com o decremento absoluto das frequências cardíacas obtidas no período de recuperação ativa após o esforço

\begin{tabular}{|c|c|c|c|c|c|c|c|c|c|c|}
\hline & \multicolumn{2}{|c|}{$\mathrm{DFC}_{1}$} & \multicolumn{2}{|c|}{$\mathrm{DFC}_{2}$} & \multicolumn{2}{|c|}{$\mathrm{DFC}_{3}$} & \multicolumn{2}{|c|}{$\mathrm{DFC}_{4}$} & \multicolumn{2}{|c|}{$\mathrm{DFC}_{5}$} \\
\hline & $\mathbf{r}_{\mathrm{s}}$ & $\mathbf{p}$ & $\mathbf{r}_{\mathrm{s}}$ & $\mathbf{p}$ & $\mathbf{r}_{\mathrm{s}}$ & $\mathbf{p}$ & $\mathbf{r}_{\mathrm{s}}$ & $\mathbf{p}$ & $\mathbf{r}_{\mathrm{s}}$ & $\mathbf{p}$ \\
\hline iRR & $-0,18$ & 0,21 & $-0,30$ & $0,03 *$ & $-0,35$ & $0,01 *$ & $-0,44$ & $0,02 *$ & $-0,55$ & $0,0001 *$ \\
\hline Média (ms) & 0,23 & 0,12 & 0,38 & $0,008^{*}$ & 0,43 & $0,003^{*}$ & 0,51 & $0,0001 *$ & 0,62 & $0,0001^{*}$ \\
\hline Desvio padrão (ms) & $-0,07$ & 0,60 & $-0,01$ & 0,92 & 0,05 & 0,70 & 0,22 & 0,13 & 0,17 & 0,25 \\
\hline r-MSSD (ms) & 0,05 & 0,69 & 0,13 & 0,35 & 0,15 & 0,31 & 0,28 & $0,05^{*}$ & 0,32 & $0,03 *$ \\
\hline
\end{tabular}

Correlação de Spearman. $\mathrm{DFC}_{1}$ a $\mathrm{DFC}_{5}$ : decremento absoluto da frequência cardíaca obtidos do primeiro ao quinto minuto de recuperação após o teste de esforço 
Tabela 8.2: Correlação $(n=46)$ entre os diversos índices temporais das séries de intervalo RR na posição ortostática previamente ao esforço com o decremento absoluto das frequências cardíacas obtidas no período de recuperação ativa após o esforço

\begin{tabular}{|c|c|c|c|c|c|c|c|c|c|c|}
\hline & \multicolumn{2}{|c|}{$\mathrm{DFC}_{1}$} & \multicolumn{2}{|c|}{$\overline{\mathrm{DFC}_{2}}$} & \multicolumn{2}{|c|}{$\overline{\mathrm{DFC}_{3}}$} & \multicolumn{2}{|c|}{$\overline{\mathrm{DFC}_{4}}$} & \multicolumn{2}{|c|}{$\overline{\mathrm{DFC}_{5}}$} \\
\hline iRR & $-0,20$ & 0,17 & $-0,28$ & $0,05 *$ & $-0,35$ & $0,01 *$ & $-0,32$ & $0,02 *$ & $-0,35$ & $0,01 *$ \\
\hline Média (ms) & 0,15 & 0,31 & 0,26 & 0,07 & 0,36 & $0,01 *$ & 0,38 & $0,009^{*}$ & 0,52 & $0,0001 *$ \\
\hline Desvio padrão (ms) & 0,08 & 0,58 & 0,11 & 0,43 & 0,21 & 0,15 & 0,20 & 0,17 & 0,31 & $0,03^{*}$ \\
\hline r-MSSD (ms) & 0,006 & 0,97 & 0,04 & 0,77 & 0,12 & 0,40 & 0,14 & 0,33 & 0,21 & 0,14 \\
\hline
\end{tabular}

Correlação de Spearman. $\mathrm{DFC}_{1}$ a $\mathrm{DFC}_{5}$ : decremento absoluto da frequência cardíaca obtidos do primeiro ao quinto minuto de recuperação após o teste de esforço 
Tabela 8.3: Correlação $(n=46)$ entre a variação relativa dos diversos índices temporais das séries de intervalo $R R$ na transição da posição supina para posição ortostática e o decremento absoluto das frequências cardíacas obtidas no período de recuperação ativa após o esforço

\begin{tabular}{|c|c|c|c|c|c|c|c|c|c|c|}
\hline & \multicolumn{2}{|c|}{$\mathrm{DFC}_{1}$} & \multicolumn{2}{|c|}{$\mathrm{DFC}_{2}$} & \multicolumn{2}{|c|}{$\mathrm{DFC}_{3}$} & \multicolumn{2}{|c|}{$\mathrm{DFC}_{4}$} & \multicolumn{2}{|c|}{$\mathrm{DFC}_{5}$} \\
\hline$\Delta \mathrm{iRR}$ & $-0,03$ & 0,80 & $-0,01$ & 0,97 & 0,02 & 0,87 & 0,10 & 0,49 & 0,11 & 0,44 \\
\hline$\Delta$ Média (ms) & 0,06 & 0,68 & 0,06 & 0,64 & 0,04 & 0,77 & 0,11 & 0,44 & 0,07 & 0,60 \\
\hline$\Delta$ Desvio padrão (ms) & $-0,20$ & 0,16 & $-0,22$ & 0,13 & $-0,21$ & 0,15 & $-0,18$ & 0,18 & $-0,19$ & 0,18 \\
\hline$\Delta \mathbf{r}-\mathbf{M S S D}(\mathrm{ms})$ & $-0,007$ & 0,96 & 0,06 & 0,68 & $-0,01$ & 0,92 & 0,08 & 0,59 & 0,06 & 0,67 \\
\hline
\end{tabular}

Correlação de Spearman. $\Delta$ : diferença relativa entre os valores obtidos nas posições supina e ortostática; $\mathrm{DFC}_{1}$ a $\mathrm{DFC}_{5}$ : decremento absoluto da frequência cardíaca obtidos do primeiro ao quinto minuto de recuperação após o teste de esforço 
Tabela 9.1: Correlação $(n=46)$ entre os diversos índices espectrais das séries de intervalo RR na posição supina previamente ao esforço com o decremento absoluto das frequências cardíacas obtidas no período de recuperação ativa após o esforço

\begin{tabular}{|c|c|c|c|c|c|c|c|c|c|c|}
\hline & \multicolumn{2}{|c|}{$\mathrm{DFC}_{1}$} & \multicolumn{2}{|c|}{$\mathrm{DFC}_{2}$} & \multicolumn{2}{|c|}{$\mathrm{DFC}_{3}$} & \multicolumn{2}{|c|}{$\mathrm{DFC}_{4}$} & \multicolumn{2}{|c|}{$\overline{\mathrm{DFC}_{5}}$} \\
\hline & $\mathbf{r}_{\mathrm{s}}$ & $\mathbf{p}$ & $\mathbf{r}_{\mathrm{s}}$ & $\mathbf{p}$ & $\mathbf{r}_{\mathrm{s}}$ & $\mathbf{p}$ & $\mathbf{r}_{\mathrm{s}}$ & $\mathbf{p}$ & $\mathbf{r}_{\mathrm{s}}$ & $\mathbf{p}$ \\
\hline Área total $\left(\mathrm{ms}^{2}\right)$ & $-0,06$ & 0,65 & 0,005 & 0,97 & 0,07 & 0,63 & 0,23 & 0,11 & 0,15 & 0,3 \\
\hline Área abs BF & $-0,09$ & 0,53 & $-0,006$ & 0,97 & $-0,06$ & 0,68 & 0,16 & 0,27 & 0,02 & 0,88 \\
\hline Área nor BF & $-0,06$ & 0,68 & $-0,10$ & 0,48 & $-0,09$ & 0,52 & $-0,18$ & 0,21 & $-0,33$ & $0,02 *$ \\
\hline Área abs AF & $-0,04$ & 0,76 & 0,05 & 0,73 & 0,08 & 0,59 & 0,24 & 0,09 & 0,23 & 0,11 \\
\hline Área nor AF & 0,06 & 0,69 & 0,10 & 0,47 & 0,09 & 0,51 & 0,18 & 0,22 & 0,33 & $0,02 *$ \\
\hline Razão BF\AF & $-0,04$ & 0,75 & $-0,09$ & 0,51 & $-0,09$ & 0,54 & $-0,18$ & 0,21 & $-0,33$ & $0,02 *$ \\
\hline
\end{tabular}

Correlação de Spearman. DFC1 a DFC5: decremento absoluto da frequência cardíaca do primeiro ao quinto minuto; abs: área absoluta; nor: área normalizada 
Tabela 9.2: Correlação $(n=46)$ entre os diversos índices espectrais das séries de intervalo RR na posição ortostática previamente ao esforço com o decremento absoluto das frequências cardíacas obtidas no período de recuperação ativa após o esforço

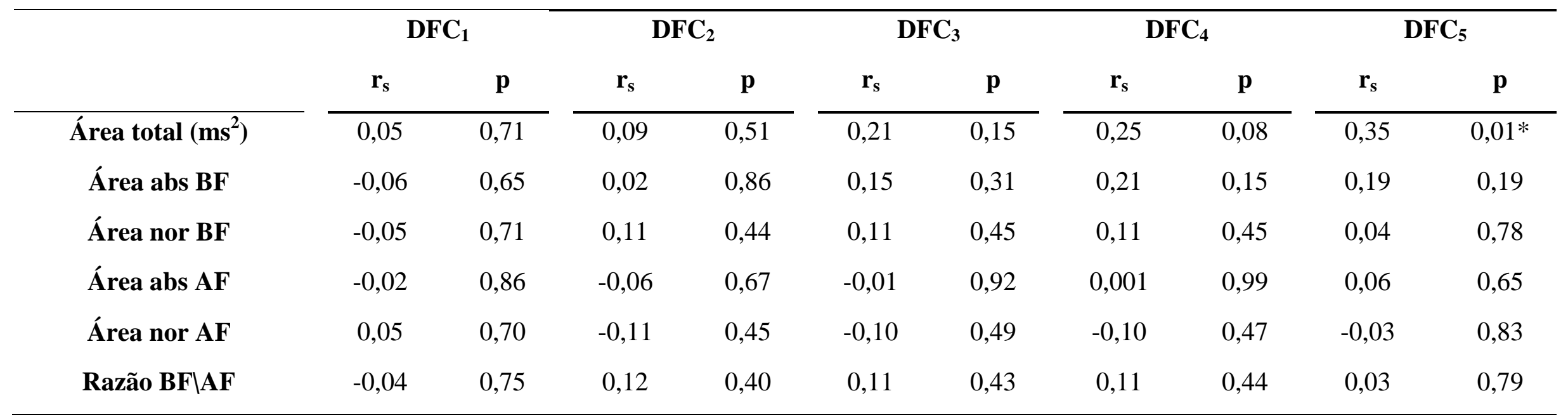

Correlação de Spearman. DFC1 a DFC5: decremento absoluto da frequência cardíaca do primeiro ao quinto minuto; abs: área absoluta; nor: área normalizada 
Tabela 9.3: Correlação $(n=46)$ entre a variação relativa dos diversos índices espectrais das séries de intervalo $R R$ na transição da posição supina para posição ortostática e o decremento absoluto das frequências cardíacas obtidas no período de recuperação ativa após o esforço

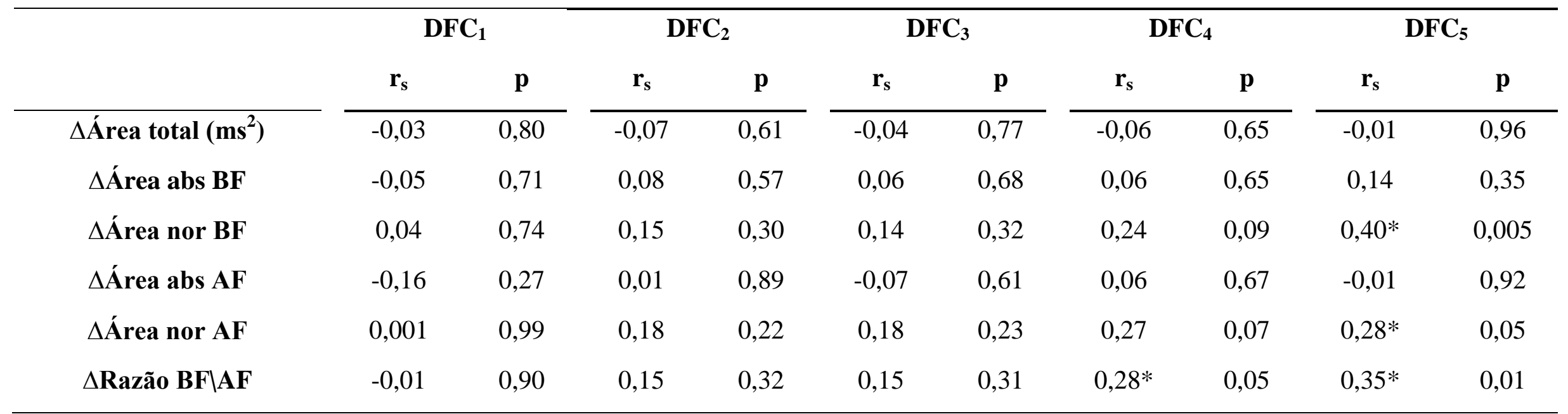

Correlação de Spearman. $\Delta$ : diferença relativa entre os valores obtidos nas posições supina e ortostática ; $\mathrm{DFC}_{1}$ a $\mathrm{DFC}_{5}$ : decremento absoluto da frequência cardíaca obtidos do primeiro ao quinto minuto de recuperação após o teste de esforço; abs: área absoluta; nor: área normalizada 
Tabela 10.1: Correlação $(n=46)$ entre os diversos índices tempo-frequenciais das séries de intervalo RR na posição supina previamente ao esforço com o decremento absoluto das frequências cardíacas obtidas no período de recuperação ativa após o esforço

\begin{tabular}{|c|c|c|c|c|c|c|c|c|c|c|}
\hline & \multicolumn{2}{|c|}{$\mathrm{DFC}_{1}$} & \multicolumn{2}{|c|}{$\mathrm{DFC}_{2}$} & \multicolumn{2}{|c|}{$\mathrm{DFC}_{3}$} & \multicolumn{2}{|c|}{$\mathrm{DFC}_{4}$} & \multicolumn{2}{|c|}{$\mathrm{DFC}_{5}$} \\
\hline & $\mathbf{r}_{\mathrm{s}}$ & $\mathbf{p}$ & $\mathbf{r}_{\mathrm{s}}$ & $\mathbf{p}$ & $\mathbf{r}_{\mathrm{s}}$ & $\mathbf{p}$ & $\mathbf{r}_{\mathrm{s}}$ & $\mathbf{p}$ & $\mathbf{r}_{\mathrm{s}}$ & $\mathbf{p}$ \\
\hline Grau & $-0,08$ & 0,58 & 0,06 & 0,66 & 0,15 & 0,3 & 0,21 & 0,15 & 0,16 & 0,28 \\
\hline Natureza & $-0,14$ & 0,35 & $-0,17$ & 0,23 & $-0,14$ & 0,35 & $-0,19$ & 0,20 & $-0,31$ & $0,03 *$ \\
\hline Área razão > 1 & $-0,08$ & 0,58 & $-0,15$ & 0,31 & $-0,15$ & 0,29 & $-0,18$ & 0,22 & $-0,30$ & $0,04 *$ \\
\hline
\end{tabular}

Correlação de Spearman. $\Delta$ : diferença relativa entre os valores obtidos nas posições supina e ortostática; DFC1 a DFC5: decremento absoluto da frequência cardíaca do primeiro ao quinto minuto; $\mathrm{CV}$ : coeficiente de variação 
Tabela 10.2: Correlação $(n=46)$ entre os diversos índices tempo-frequenciais das séries de intervalo RR na posição ortostática previamente ao esforço com o decremento absoluto das frequências cardíacas obtidas no período de recuperação ativa após o esforço

\begin{tabular}{|c|c|c|c|c|c|c|c|c|c|c|}
\hline & \multicolumn{2}{|c|}{$\mathrm{DFC}_{1}$} & \multicolumn{2}{|c|}{$\mathrm{DFC}_{2}$} & \multicolumn{2}{|c|}{$\mathrm{DFC}_{3}$} & \multicolumn{2}{|c|}{$\mathrm{DFC}_{4}$} & \multicolumn{2}{|c|}{$\mathrm{DFC}_{5}$} \\
\hline & $\mathbf{r}_{\mathrm{s}}$ & $\mathbf{p}$ & $\mathbf{r}_{\mathrm{s}}$ & $\mathbf{p}$ & $\mathbf{r}_{\mathrm{s}}$ & $\mathbf{p}$ & $\mathbf{r}_{\mathrm{s}}$ & $\mathbf{p}$ & $\mathbf{r}_{\mathrm{s}}$ & $\mathbf{p}$ \\
\hline Grau & 0,08 & 0,56 & 0,07 & 0,61 & 0,22 & 0,13 & 0,24 & 0,09 & 0,29 & $0,04 *$ \\
\hline Natureza & 0,07 & 0,61 & 0,16 & 0,28 & 0,19 & 0,18 & 0,20 & 0,17 & 0,11 & 0,44 \\
\hline Área razão > 1 & 0,08 & 0,59 & 0,19 & 0,19 & 0,16 & 0,28 & 0,18 & 0,21 & 0,12 & 0,39 \\
\hline Área razão < 1 & 0,09 & 0,54 & $-0,05$ & 0,70 & $-0,13$ & 0,36 & $-0,13$ & 0,38 & $-0,05$ & 0,97 \\
\hline CV da razão & 0,26 & 0,11 & 0,25 & 0,13 & 0,24 & 0,15 & 0,27 & 0,10 & 0,26 & 0,12 \\
\hline
\end{tabular}

Correlação de Spearman. DFC1 a DFC5: decremento absoluto da frequência cardíaca do primeiro ao quinto minuto; CV: coeficiente de variação 
Tabela 10.3: Correlação $(n=46)$ entre os diversos índices tempo-frequenciais das séries de intervalo RR na transição da posição supina para posição ortostática previamente ao esforço e o decremento absoluto das frequências cardíacas obtidas no período de recuperação ativa após o esforço

\begin{tabular}{|c|c|c|c|c|c|c|c|c|c|c|}
\hline & \multicolumn{2}{|c|}{$\mathrm{DFC}_{1}$} & \multicolumn{2}{|c|}{$\mathrm{DFC}_{2}$} & \multicolumn{2}{|c|}{$\mathrm{DFC}_{3}$} & \multicolumn{2}{|c|}{$\mathrm{DFC}_{4}$} & \multicolumn{2}{|c|}{$\mathrm{DFC}_{5}$} \\
\hline & $\mathbf{r}_{\mathrm{s}}$ & $\mathbf{p}$ & $\mathbf{r}_{\mathrm{s}}$ & $\mathbf{p}$ & $\mathbf{r}_{\mathrm{s}}$ & $\mathbf{p}$ & $\mathbf{r}_{\mathrm{s}}$ & $\mathbf{p}$ & $\mathbf{r}_{\mathrm{s}}$ & $\mathbf{p}$ \\
\hline$\Delta$ Grau & 0,01 & 0,99 & $-0,16$ & 0,27 & $-0,15$ & 0,29 & $-0,05$ & 0,71 & $-0,11$ & 0,45 \\
\hline$\Delta$ Natureza & 0,19 & 0,19 & 0,31 & $0,03^{*}$ & 0,29 & $0,04 *$ & 0,32 & $0,02 *$ & 0,39 & $0,006^{*}$ \\
\hline$\Delta$ Área razão > 1 & 0,24 & 0,10 & 0,33 & $0,02 *$ & 0,32 & $0,03 *$ & 0,37 & $0,01 *$ & 0,46 & $0,01^{*}$ \\
\hline
\end{tabular}

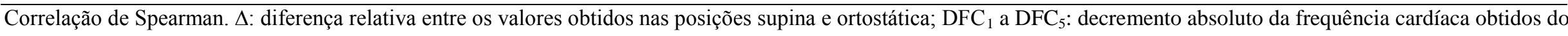
primeiro ao quinto minuto de recuperação após o teste de esforço; CV: coeficiente de recuperação 
Tabela 11.1: Correlação $(n=46)$ entre os diversos índices de Poincaré na posição supina previamente ao esforço com o decremento absoluto das frequências cardíacas obtidas no período de recuperação ativa após o esforço

\begin{tabular}{|c|c|c|c|c|c|c|c|c|c|c|}
\hline & \multicolumn{2}{|c|}{$\mathrm{DFC}_{1}$} & \multicolumn{2}{|c|}{$\mathrm{DFC}_{2}$} & \multicolumn{2}{|c|}{$\mathbf{D F C}_{3}$} & \multicolumn{2}{|c|}{$\mathrm{DFC}_{4}$} & \multicolumn{2}{|c|}{$\mathrm{DFC}_{5}$} \\
\hline & $\mathbf{r}_{\mathrm{s}}$ & $\mathbf{p}$ & $\mathbf{r}_{\mathrm{s}}$ & $\mathbf{p}$ & $\mathbf{r}_{\mathrm{s}}$ & $\mathbf{p}$ & $\mathbf{r}_{\mathrm{s}}$ & $\mathbf{p}$ & $\mathbf{r}_{\mathrm{s}}$ & $\mathbf{p}$ \\
\hline $\mathbf{N}^{\circ}$ de pontos & $-0,14$ & 0,44 & $-0,26$ & 0,07 & $-0,33$ & $0,02 *$ & $-0,41$ & $0,04 *$ & $-0,55$ & $0,0001^{*}$ \\
\hline Centróide (ms) & 0,23 & 0,12 & 0,38 & $0,008^{*}$ & 0,42 & $0,03 *$ & 0,50 & $0,0001 *$ & 0,61 & $0,0001 *$ \\
\hline SD1 & 0,09 & 0,53 & 0,16 & 0,27 & 0,15 & 0,29 & 0,26 & 0,07 & 0,29 & $0,04 *$ \\
\hline Área da elipse (ms2) & 0,02 & 0,87 & 0,11 & 0,44 & 0,15 & 0,29 & 0,29 & $0,04 *$ & 0,25 & 0,09 \\
\hline Coeficiente de correlação & $-0,29$ & $0,04 *$ & $-0,34$ & $0,01 *$ & $-0,26$ & 0,07 & $-0,25$ & 0,08 & $-0,39$ & $0,007 *$ \\
\hline
\end{tabular}


Tabela 11.2: Correlação $(n=46)$ entre os diversos índices de Poincaré na posição ortostática previamente ao esforço com o decremento absoluto das frequências cardíacas obtidas no período de recuperação ativa após o esforço

\begin{tabular}{|c|c|c|c|c|c|c|c|c|c|c|}
\hline & \multicolumn{2}{|c|}{$\mathrm{DFC}_{1}$} & \multicolumn{2}{|c|}{$\mathrm{DFC}_{2}$} & \multicolumn{2}{|c|}{$\mathrm{DFC}_{3}$} & \multicolumn{2}{|c|}{$\mathrm{DFC}_{4}$} & \multicolumn{2}{|c|}{$\mathrm{DFC}_{5}$} \\
\hline & $\mathbf{r}_{\mathrm{s}}$ & $\mathbf{p}$ & $\mathbf{r}_{\mathrm{s}}$ & $\mathbf{p}$ & $\mathbf{r}_{\mathrm{s}}$ & $\mathbf{p}$ & $\mathbf{r}_{\mathrm{s}}$ & $\mathbf{p}$ & $\mathbf{r}_{\mathrm{s}}$ & $\mathbf{p}$ \\
\hline $\mathrm{N}^{\circ}$ de pontos & $-0,07$ & 0,63 & $-0,17$ & 0,24 & $-0,29$ & $0,04 *$ & $-0,19$ & 0,20 & $-0,24$ & 0,09 \\
\hline Centróide (ms) & 0,14 & 0,33 & 0,25 & 0,08 & 0,35 & $0,01 *$ & 0,37 & $0,01 *$ & 0,50 & $0,0001 *$ \\
\hline SD1 & $-0,01$ & 0,90 & 0,01 & 0,90 & 0,08 & 0,55 & 0,11 & 0,47 & 0,19 & 0,18 \\
\hline Área da elipse (ms2) & 0,02 & 0,86 & 0,06 & 0,66 & 0,14 & 0,34 & 0,15 & 0,31 & 0,25 & 0,09 \\
\hline Coeficiente de correlação & 0,16 & 0,27 & 0,16 & 0,28 & 0,11 & 0,46 & 0,05 & 0,73 & 0,003 & 0,98 \\
\hline
\end{tabular}

Correlação de Spearman. $\mathrm{DFC}_{1}$ a $\mathrm{DFC}_{5}$ : decrementos absolutos das frequências cardíacas obtidos do primeiro ao quinto minuto de recuperação após o teste de esforço 
Tabela 11.3: Correlação $(n=46)$ entre os diversos índices de Poincaré na transição da posição supina para posição ortostática previamente ao esforço e o decremento absoluto das frequências cardíacas obtidas no período de recuperação ativa após o esforço

\begin{tabular}{|c|c|c|c|c|c|c|c|c|c|c|}
\hline & \multicolumn{2}{|c|}{$\mathrm{DFC}_{1}$} & \multicolumn{2}{|c|}{$\mathrm{DFC}_{2}$} & \multicolumn{2}{|c|}{$\mathrm{DFC}_{3}$} & \multicolumn{2}{|c|}{$\mathrm{DFC}_{4}$} & \multicolumn{2}{|c|}{$\mathrm{DFC}_{5}$} \\
\hline & $\mathbf{r}_{\mathrm{s}}$ & $\mathbf{p}$ & $\mathbf{r}_{\mathrm{s}}$ & $\mathbf{p}$ & $\mathbf{r}_{\mathrm{s}}$ & $\mathbf{p}$ & $\mathbf{r}_{\mathrm{s}}$ & $\mathbf{p}$ & $\mathbf{r}_{\mathrm{s}}$ & $\mathbf{p}$ \\
\hline$\Delta \mathbf{N}^{\circ}$ de pontos & 0,08 & 0,59 & 0,06 & 0,65 & 0,02 & 0,85 & 0,18 & 0,22 & 0,19 & 0,2 \\
\hline$\Delta$ Centróide (ms) & 0,06 & 0,66 & 0,1 & 0,5 & 0,05 & 0,71 & 0,06 & 0,65 & 0,03 & 0,84 \\
\hline$\Delta$ SD1 & 0,009 & 0,95 & 0,04 & 0,76 & $-0,01$ & 0,95 & 0,17 & 0,25 & 0,12 & 0,39 \\
\hline$\Delta$ Área da elipse (ms2) & $-0,15$ & 0,32 & $-0,09$ & 0,54 & $-0,12$ & 0,4 & 0,09 & 0,51 & $-0,01$ & 0,91 \\
\hline$\Delta$ Coeficiente de correlação & 0,27 & 0,06 & 0,32 & $0,02 *$ & 0,22 & 0,12 & 0,17 & 0,25 & 0,31 & $0,03 *$ \\
\hline
\end{tabular}

Correlação de Spearman. $\mathrm{DFC}_{1}$ a $\mathrm{DFC}_{5}$ : decrementos absolutos das frequências cardíacas obtidos do primeiro ao quinto minuto de recuperação após o teste de esforço; $\Delta$ : diferença relativa entre os valores obtidos nas posições supina e ortostática 


\section{4-Correlação entre a variabilidade da frequência cardíaca em repouso e o coeficiente de recuperação da frequência cardíaca}

$\mathrm{Na}$ análise temporal, não foram observadas correlações significativas entre os índices registrados na posição supina e o decremento da frequência cardíaca (Tabela 12.1). Na posição ortostática, foi observada uma correlação negativa entre o número de iRR e o CR no quinto minuto de recuperação $\left(\mathrm{r}_{\mathrm{s}}-0,28 \mathrm{p}=0,05\right)$ e entre a média de iRR e o CR no quinto minuto pós esforço $\left(\mathrm{r}_{\mathrm{s}} 0,29 \mathrm{p}=0,05\right)$ (Tabela 12.2). Conforme apresentado na Tabela 12.3, foram observadas correlações significativas entre a variação relativa da média de iRR na transição supino-ortostático e o CR no terceiro $\left(\mathrm{r}_{\mathrm{s}}-0,29 \mathrm{p}=0,04\right)$ e quarto $\left(\mathrm{r}_{\mathrm{s}}-0,29 \mathrm{p}=0,05\right)$ minuto de recuperação..

No domínio espectral, não foram observadas correlações significativas entre o CR e a variabilidade da frequência nas posições supina (Tabela 13.1) e ortostática (Tabela 13.2). Na transição supino-ortostático foram observadas correlações entre a variação relativa da área total e o CR no quarto $\left(\mathrm{r}_{\mathrm{s}} 0,28 \mathrm{p}=0,05\right)$ e quinto $\left(\mathrm{r}_{\mathrm{s}} 0,40 \mathrm{p}=0,005\right)$ minuto do período de recuperação ativa (Tabela 13.3).

Quanto à análise tempo-frequencial, não foram observadas correlações entre os diferentes índices avaliados nas posições supina (Tabela 14.1) e ortostática (Tabela 14.2), ou na transição supino-ortostático (Tabela 14.3), e o CR em qualquer momento do protocolo de recuperação.

Na plotagem de Poincaré, não foram observadas correlações significativas entre os índices avaliados na posição supina e o CR, dados apresentados na tabela 15.1. Durante o ortostatismo, correlações foram encontradas entre o centróide e o CR no quinto minuto de recuperação $\left(\mathrm{r}_{\mathrm{s}} 0,30 \mathrm{p}=0,04\right)$. Quando analisada a variação dos índices de Poincaré diante da mudança postural, foi observada correlação significativa apenas entre a variação relativa do centróide e o CR no quinto minuto pós esforço $\left(r_{s}-0,28\right.$ p = 0,05), dados apresentados na Tabela 15.3. 
Tabela 12.1: Correlação $(n=46)$ entre os diversos índices temporais das séries de intervalos $R R$ na posição supina previamente ao esforço com o coeficiente de recuperação da frequência cardíaca

\begin{tabular}{|c|c|c|c|c|c|c|c|c|c|c|}
\hline & \multicolumn{2}{|c|}{$\mathbf{C R}_{1}$} & \multicolumn{2}{|c|}{$\mathbf{C R}_{2}$} & \multicolumn{2}{|c|}{$\mathbf{C R}_{\mathbf{3}}$} & \multicolumn{2}{|c|}{$\mathbf{C R}_{4}$} & \multicolumn{2}{|c|}{$\mathrm{CR}_{5}$} \\
\hline iRR & 0,07 & 0,63 & $-0,01$ & 0,93 & $-0,01$ & 0,91 & 0,01 & 0,91 & $-0,12$ & 0,40 \\
\hline Média (ms) & $-0,05$ & 0,70 & 0,05 & 0,7 & 0,03 & 0,80 & $-0,02$ & 0,87 & 0,08 & 0,56 \\
\hline Desvio padrão (ms) & $-0,15$ & 0,30 & $-0,16$ & 0,27 & $-0,14$ & 0,33 & 0,02 & 0,88 & $-0,16$ & 0,28 \\
\hline pNN50 (\%) & $-0,12$ & 0,40 & $-0,13$ & 0,37 & $-0,21$ & 0,14 & $-0,16$ & 0,28 & $-0,14$ & 0,32 \\
\hline r-MSSD (ms) & $-0,11$ & 0,43 & $-0,11$ & 0,44 & $-0,19$ & 0,18 & $-0,09$ & 0,53 & $-0,13$ & 0,37 \\
\hline
\end{tabular}

Correlação de Spearman. CR1 a CR5: coeficiente de recuperação calculado do primeiro ao quinto minuto do protocolo de recuperação ativa 
Tabela 12.2: Correlação $(n=46)$ entre os diversos índices temporais das séries de intervalos RR na posição ortostática previamente ao esforço e o coeficiente de recuperação da frequência cardíaca

\begin{tabular}{|c|c|c|c|c|c|c|c|c|c|c|}
\hline & \multicolumn{2}{|c|}{$\mathbf{C R}_{1}$} & \multicolumn{2}{|c|}{$\mathbf{C R}_{\mathbf{2}}$} & \multicolumn{2}{|c|}{$\mathbf{C R}_{\mathbf{3}}$} & \multicolumn{2}{|c|}{$\mathbf{C R}_{4}$} & \multicolumn{2}{|c|}{$\mathbf{C R}_{5}$} \\
\hline & $\mathbf{r}_{\mathrm{s}}$ & $\mathbf{p}$ & $\mathbf{r}_{\mathrm{s}}$ & $\mathbf{p}$ & $\mathbf{r}_{\mathrm{s}}$ & $\mathbf{p}$ & $\mathbf{r}_{\mathrm{s}}$ & $\mathbf{p}$ & $\mathbf{r}_{\mathrm{s}}$ & $\mathbf{p}$ \\
\hline Média (ms) & 0,001 & 0,99 & 0,14 & 0,34 & 0,23 & 0,10 & 0,15 & 0,31 & 0,29 & $0,05^{*}$ \\
\hline Desvio padrão (ms) & 0,04 & 0,75 & $-0,01$ & 0,90 & 0,07 & 0,60 & $-0,002$ & 0,98 & 0,05 & 0,71 \\
\hline pNN50 (\%) & 0,01 & 0,91 & $-0,06$ & 0,68 & 0,06 & 0,65 & 0,02 & 0,86 & 0,07 & 0,64 \\
\hline r-MSSD (ms) & 0,02 & 0,87 & $-0,02$ & 0,88 & 0,09 & 0,53 & 0,07 & 0,63 & 0,10 & 0,50 \\
\hline
\end{tabular}

Correlação de Spearman. CR1 a CR5: coeficiente de recuperação calculado do primeiro ao quinto minuto do protocolo de recuperação ativa 
Tabela 12.3: Correlação $(n=46)$ entre os diversos índices temporais das séries de intervalos $R R$ na transição da posição supina para posição ortostática previamente ao esforço e o coeficiente de recuperação da frequência cardíaca

\begin{tabular}{|c|c|c|c|c|c|c|c|c|c|c|}
\hline & \multicolumn{2}{|c|}{$\mathbf{C R}_{1}$} & \multicolumn{2}{|c|}{$\mathbf{C R}_{2}$} & \multicolumn{2}{|c|}{$\mathbf{C R}_{3}$} & \multicolumn{2}{|c|}{$\mathbf{C R}_{4}$} & \multicolumn{2}{|c|}{$\mathbf{C R}_{5}$} \\
\hline & $\mathbf{r}_{\mathrm{s}}$ & $\mathbf{p}$ & $\mathbf{r}_{\mathrm{s}}$ & $\mathbf{p}$ & $\mathbf{r}_{\mathrm{s}}$ & $\mathbf{p}$ & $\mathbf{r}_{\mathrm{s}}$ & $\mathbf{p}$ & $\mathbf{r}_{\mathrm{s}}$ & $\mathbf{p}$ \\
\hline$\Delta$ Média (ms) & $-0,12$ & 0,42 & $-0,20$ & 0,16 & $-0,29$ & $0,04^{*}$ & $-0,29$ & $0,05^{*}$ & $-0,32$ & 0,09 \\
\hline$\Delta$ Desvio padrão (ms) & $-0,01$ & 0,90 & 0,01 & 0,93 & 0,02 & 0,86 & 0,10 & 0,48 & 0,13 & 0,36 \\
\hline$\Delta p N N 50(\%)$ & 0,02 & 0,85 & 0,19 & 0,19 & $-0,02$ & 0,85 & 0,10 & 0,46 & $-0,02$ & 88 \\
\hline$\Delta \mathbf{r}-\mathbf{M S S D}(\mathbf{m s})$ & $-0,12$ & 0,41 & $-0,06$ & 0,67 & $-0,23$ & 0,10 & $-0,13$ & 0,37 & $-0,18$ & 0,21 \\
\hline
\end{tabular}

Correlação de Spearman. CR1 a CR5: coeficiente de recuperação calculado do primeiro ao quinto minuto do protocolo de recuperação ativa; $\Delta$ : diferença relativa entre os valores obtidos nas posições supina e ortostática 
Tabela 13.1: Correlação $(n=46)$ entre os diversos índices espectrais das séries de intervalos RR na posição supina previamente ao esforço e o coeficiente de recuperação da frequência cardíaca

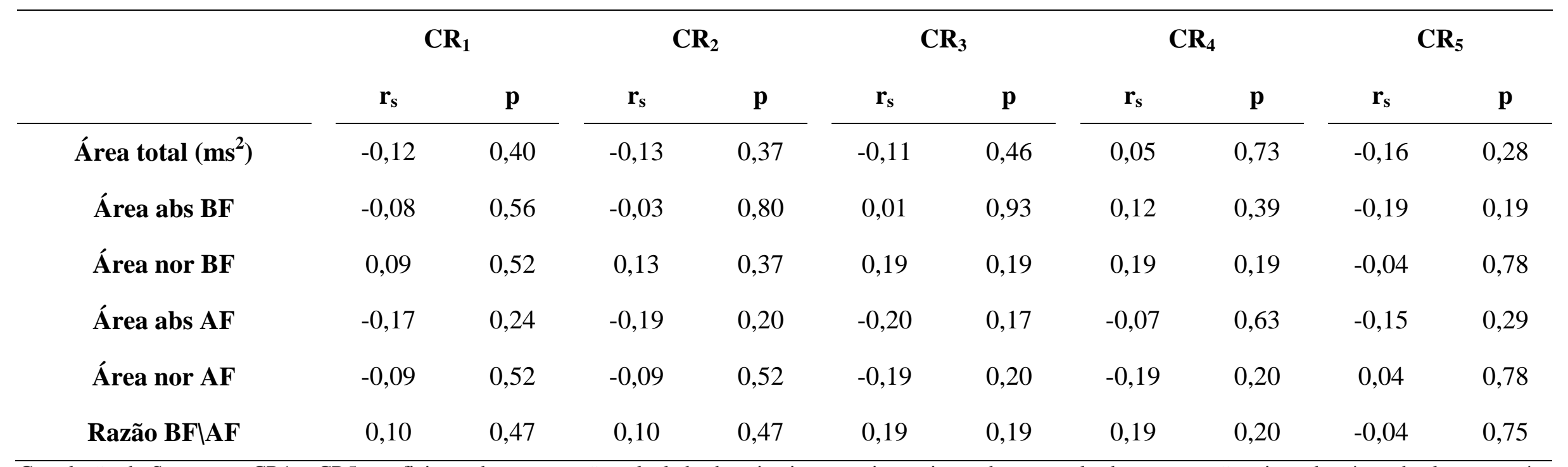

Correlação de Spearman. CR1 a CR5: coeficiente de recuperação calculado do primeiro ao quinto minuto do protocolo de recuperação ativa; abs: área absoluta; nor: área normalizada 
Tabela 13.2: Correlação $(n=46)$ entre os diversos índices espectrais das séries de intervalos RR na posição ortostática previamente ao esforço e o coeficiente de recuperação da frequência cardíaca

\begin{tabular}{|c|c|c|c|c|c|c|c|c|c|c|}
\hline & \multicolumn{2}{|c|}{$\mathbf{C R}_{1}$} & \multicolumn{2}{|c|}{$\mathbf{C R}_{2}$} & \multicolumn{2}{|c|}{$\mathbf{C R}_{\mathbf{3}}$} & \multicolumn{2}{|c|}{$\mathbf{C R}_{4}$} & \multicolumn{2}{|c|}{$\mathbf{C R}_{5}$} \\
\hline & $\mathbf{r}_{\mathrm{s}}$ & $\mathbf{p}$ & $\mathbf{r}_{\mathrm{s}}$ & $\mathbf{p}$ & $\mathbf{r}_{\mathrm{s}}$ & $\mathbf{p}$ & $\mathbf{r}_{\mathrm{s}}$ & $\mathbf{p}$ & $\mathbf{r}_{\mathrm{s}}$ & $\mathbf{p}$ \\
\hline Área abs BF & $-0,04$ & 0,75 & $-0,03$ & 0,82 & 0,08 & 0,57 & 0,04 & 0,78 & 0,003 & 0,98 \\
\hline Área nor BF & $-0,06$ & 0,65 & 0,16 & 0,26 & 0,10 & 0,47 & 0,08 & 0,58 & 0,06 & 0,65 \\
\hline Área nor AF & 0,06 & 0,65 & $-0,17$ & 0,25 & $-0,10$ & 0,48 & $-0,08$ & 0,56 & $-0,06$ & 0,66 \\
\hline Razão BF\AF & $-0,06$ & 0,65 & 0,17 & 0,24 & 0,10 & 0,47 & 0,08 & 0,58 & 0,05 & 0,70 \\
\hline
\end{tabular}


Tabela 13.3: Correlação $(n=46)$ entre os diversos índices espectrais das séries de intervalos $R R$ na transição da posição supina para posição ortostática previamente ao esforço e o coeficiente de recuperação da frequência cardíaca

\begin{tabular}{|c|c|c|c|c|c|c|c|c|c|c|}
\hline & \multicolumn{2}{|c|}{$\mathbf{C R}_{1}$} & \multicolumn{2}{|c|}{$\mathbf{C R}_{2}$} & \multicolumn{2}{|c|}{$\mathbf{C R}_{\mathbf{3}}$} & \multicolumn{2}{|c|}{$\mathbf{C R}_{\mathbf{4}}$} & \multicolumn{2}{|c|}{$\mathbf{C R}_{5}$} \\
\hline$\Delta$ Área total $\left(\mathrm{ms}^{2}\right)$ & 0,15 & 0,30 & 0,15 & 0,29 & 0,22 & 0,12 & 0,28 & $0,05^{*}$ & 0,40 & $0,005^{*}$ \\
\hline$\Delta$ Área abs BF & 0,03 & 0,83 & 0,23 & 0,11 & 0,19 & 0,19 & 0,13 & 0,38 & 0,28 & $0,05^{*}$ \\
\hline$\Delta$ Área nor BF & $-0,14$ & 0,32 & $-0,09$ & 0,54 & $-0,20$ & 0,17 & $-0,20$ & 0,17 & 0,04 & 0,74 \\
\hline$\Delta$ Área nor AF & $-0,14$ & 0,33 & 0,03 & 0,82 & $-0,09$ & 0,53 & $-0,07$ & 0,63 & 0,05 & 0,70 \\
\hline$\Delta$ Razão BF\AF & $-0,20$ & 0,16 & $-0,06$ & 0,66 & $-0,17$ & 0,23 & $-0,15$ & 0,29 & 0,02 & 0,86 \\
\hline
\end{tabular}

Correlação de Spearman. CR1 a CR5: coeficiente de recuperação calculado do primeiro ao quinto minuto do protocolo de recuperação ativa; abs: área absoluta; nor: área normalizada; $\Delta$ : diferença relativa entre os valores obtidos nas posições supina e ortostática 
Tabela 14.1: Correlação $(n=46)$ entre os diversos índices tempo-frequenciais das séries de intervalos RR na posição supina previamente ao esforço e o coeficiente de recuperação da frequência cardíaca

\begin{tabular}{|c|c|c|c|c|c|c|c|c|c|c|}
\hline & \multicolumn{2}{|c|}{$\mathrm{CR}_{1}$} & \multicolumn{2}{|c|}{$\mathbf{C R}_{2}$} & \multicolumn{2}{|c|}{$\mathbf{C R}_{3}$} & \multicolumn{2}{|c|}{$\mathbf{C R}_{4}$} & \multicolumn{2}{|c|}{$\mathbf{C R}_{5}$} \\
\hline & $\mathbf{r}_{\mathrm{s}}$ & $\mathbf{p}$ & $\mathbf{r}_{\mathrm{s}}$ & $\mathbf{p}$ & $\mathbf{r}_{\mathrm{s}}$ & $\mathbf{p}$ & $\mathbf{r}_{\mathrm{s}}$ & $\mathbf{p}$ & $\mathbf{r}_{\mathrm{s}}$ & $\mathbf{p}$ \\
\hline Grau & $-0,13$ & 0,37 & $-0,07$ & 0,64 & $-0,003$ & 0,98 & 0,10 & 0,49 & $-0,05$ & 0,73 \\
\hline Natureza & 0,02 & 0,88 & 0,05 & 0,71 & 0,12 & 0,39 & 0,10 & 0,50 & $-0,07$ & 0,62 \\
\hline Área razão > 1 & 0,10 & 0,50 & 0,14 & 0,34 & 0,18 & 0,23 & 0,18 & 0,22 & $-0,004$ & 0,97 \\
\hline Área razão < 1 & $-0,03$ & 0,84 & $-0,10$ & 0,50 & $-0,12$ & 0,41 & $-0,11$ & 0,46 & 0,08 & 0,57 \\
\hline CV da razão & 0,10 & 0,50 & 0,01 & 0,93 & 0,05 & 0,71 & 0,08 & 0,56 & 0,14 & 0,33 \\
\hline
\end{tabular}

Correlação de Spearman. CR1 a CR5: coeficiente de recuperação calculado do primeiro ao quinto minuto do protocolo de recuperação ativa; CV: coeficiente de variação 
Tabela 14.2: Correlação $(n=46)$ entre os diversos índices tempo-frequenciais das séries de intervalos RR na posição ortostática previamente ao esforço e o coeficiente de recuperação da frequência cardíaca

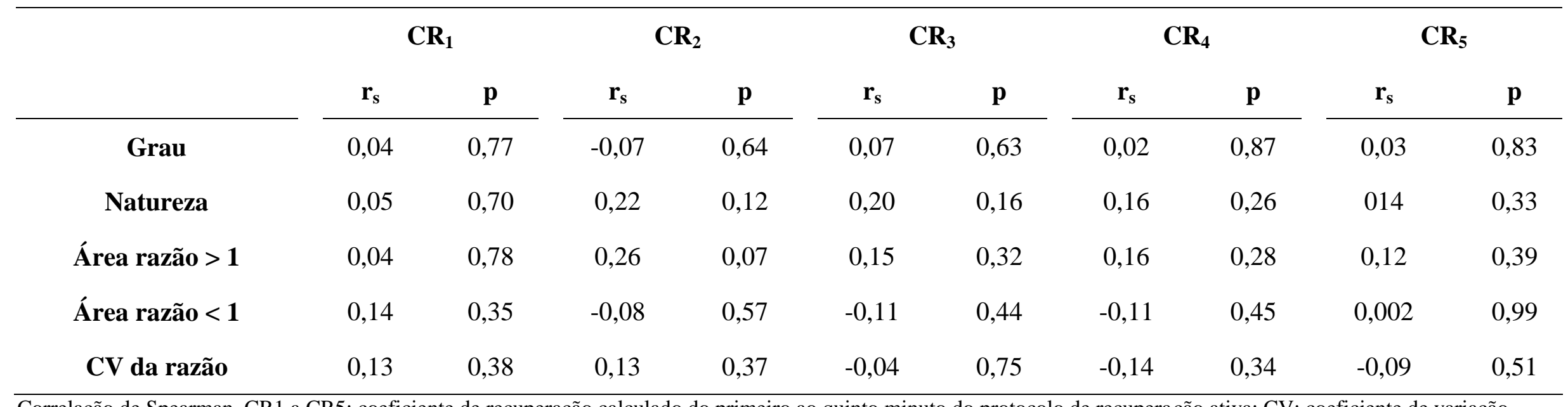

Correlação de Spearman. CR1 a CR5: coeficiente de recuperação calculado do primeiro ao quinto minuto do protocolo de recuperação ativa; CV: coeficiente de variação 
Tabela 14.3: Correlação $(n=46)$ entre os diversos índices tempo-frequenciais das séries de intervalos RR na transição da posição supina para posição ortostática previamente ao esforço e o coeficiente de recuperação da frequência cardíaca

\begin{tabular}{|c|c|c|c|c|c|c|c|c|c|c|}
\hline & \multicolumn{2}{|c|}{$\mathbf{C R}_{1}$} & \multicolumn{2}{|c|}{$\mathbf{C R}_{2}$} & \multicolumn{2}{|c|}{$\mathbf{C R}_{\mathbf{3}}$} & \multicolumn{2}{|c|}{$\mathbf{C R}_{\mathbf{4}}$} & \multicolumn{2}{|c|}{$\mathbf{C R}_{5}$} \\
\hline & $\mathbf{r}_{\mathrm{s}}$ & $\mathbf{p}$ & $\mathbf{r}_{\mathrm{s}}$ & $\mathbf{p}$ & $\mathbf{r}_{\mathrm{s}}$ & $\mathbf{p}$ & $\mathbf{r}_{\mathrm{s}}$ & $\mathbf{p}$ & $\mathbf{r}_{\mathrm{s}}$ & $\mathbf{p}$ \\
\hline$\Delta$ Natureza & 0,007 & 0,96 & 0,11 & 0,45 & 0,007 & 0,96 & $-0,005$ & 0,97 & 0,17 & 0,26 \\
\hline$\Delta$ Área razão $>1$ & 0,03 & 0,84 & 0,08 & 0,58 & $-0,02$ & 0,88 & $-0,008$ & 0,95 & 0,19 & 0,19 \\
\hline$\Delta C V$ da razão & 0,10 & 0,48 & $-0,04$ & 0,79 & 0,007 & 0,96 & $-0,13$ & 0,39 & $-0,19$ & 0,19 \\
\hline
\end{tabular}

Correlação de Spearman. CR1 a CR5: coeficiente de recuperação calculado do primeiro ao quinto minuto do protocolo de recuperação ativa; CV: coeficiente de variação; $\Delta$ : diferença relativa entre os valores obtidos nas posições supina e ortostática 
Tabela 15.1: Correlação $(n=46)$ entre os diversos índices de Poincaré na posição supina previamente ao esforço e o coeficiente de recuperação da frequência cardíaca

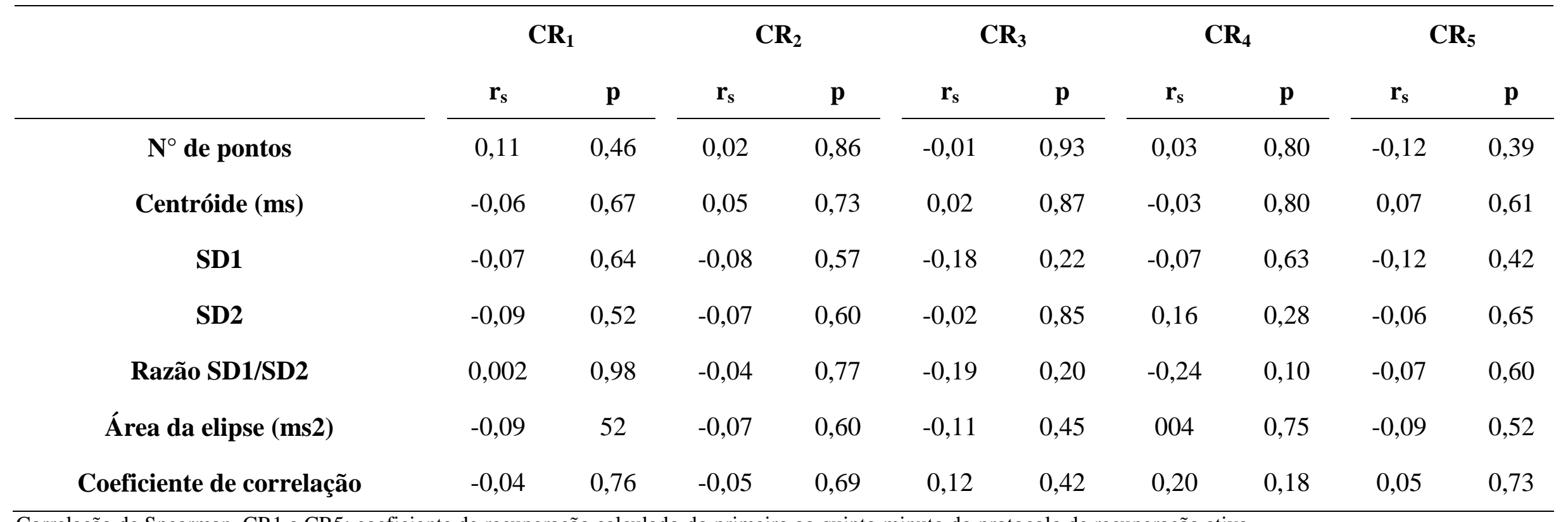


Tabela 15.2: Correlação $(n=46)$ entre os diversos índices de Poincaré na posição ortostática previamente ao esforço e o coeficiente de recuperação da frequência cardíaca

\begin{tabular}{|c|c|c|c|c|c|c|c|c|c|c|}
\hline & \multicolumn{2}{|c|}{$\mathbf{C R}_{1}$} & \multicolumn{2}{|c|}{$\mathbf{C R}_{2}$} & \multicolumn{2}{|c|}{$\mathbf{C R}_{3}$} & \multicolumn{2}{|c|}{$\mathbf{C R}_{4}$} & \multicolumn{2}{|c|}{$\mathbf{C R}_{5}$} \\
\hline & $\mathbf{r}_{\mathrm{s}}$ & $\mathbf{p}$ & $\mathbf{r}_{\mathrm{s}}$ & $\mathbf{p}$ & $\mathbf{r}_{\mathrm{s}}$ & $\mathbf{p}$ & $\mathbf{r}_{\mathrm{s}}$ & $\mathbf{p}$ & $\mathbf{r}_{\mathrm{s}}$ & $\mathbf{p}$ \\
\hline Centróide (ms) & $-0,001$ & 0,99 & 0,15 & 0,31 & 0,24 & 0,09 & 0,16 & 0,26 & 0,30 & $0,04 *$ \\
\hline SD1 & $-0,04$ & 0,97 & $-0,04$ & 0,75 & 0,03 & 0,79 & 0,05 & 0,72 & 0,07 & 0,60 \\
\hline Razão SD1/SD2 & $-0,07$ & 0,64 & $-0,10$ & 0,50 & $-0,02$ & 0,87 & 0,05 & 0,70 & 0,01 & 0,91 \\
\hline Área da elipse (ms2) & 0,01 & 0,91 & $-0,02$ & 0,87 & 0,05 & 0,70 & 0,03 & 0,82 & 0,08 & 0,57 \\
\hline Coeficiente de correlação & 0,07 & 0,62 & 0,11 & 0,43 & 0,01 & 0,91 & $-0,05$ & 0,71 & $-0,04$ & 0,74 \\
\hline
\end{tabular}


Tabela 15.3: Correlação $(n=46)$ entre os diversos índices de Poincaré na transição da posição supina para posição ortostática previamente ao esforço e o coeficiente de recuperação da frequência cardíaca

\begin{tabular}{|c|c|c|c|c|c|c|c|c|c|c|}
\hline & \multicolumn{2}{|c|}{$\mathbf{C R}_{1}$} & \multicolumn{2}{|c|}{$\mathbf{C R}_{2}$} & \multicolumn{2}{|c|}{$\mathbf{C R}_{3}$} & \multicolumn{2}{|c|}{$\mathrm{CR}_{4}$} & \multicolumn{2}{|c|}{$\mathbf{C R}_{5}$} \\
\hline & $\mathbf{r}_{\mathrm{s}}$ & $\mathbf{p}$ & $\mathbf{r}_{\mathrm{s}}$ & $\mathbf{p}$ & $\mathbf{r}_{\mathrm{s}}$ & $\mathbf{p}$ & $\mathbf{r}_{\mathrm{s}}$ & $\mathbf{p}$ & $\mathbf{r}_{\mathrm{s}}$ & $\mathbf{p}$ \\
\hline$\Delta$ Centróide (ms) & $-0,08$ & 0,57 & $-0,14$ & 0,35 & $-0,23$ & 0,12 & $-0,25$ & 0,09 & $-0,28$ & $0,05^{*}$ \\
\hline$\Delta$ SD1 & $-0,13$ & 0,37 & $-0,08$ & 0,59 & $-0,25$ & 0,08 & $-0,14$ & 0,32 & $-0,22$ & 0,12 \\
\hline$\Delta$ Razão SD1/SD2 & 0,05 & 0,72 & 0,08 & 0,58 & $-0,09$ & 0,54 & $-0,16$ & 0,26 & 0,007 & 0,96 \\
\hline$\Delta$ Área da elipse (ms2) & $-0,17$ & 0,24 & $-0,04$ & 0,76 & $-0,18$ & 0,22 & 0,02 & 0,88 & $-0,22$ & 0,14 \\
\hline$\Delta$ Coeficiente de correlação & 0,06 & 0,69 & 0,07 & 0,63 & $-0,10$ & 0,49 & $-0,17$ & 0,25 & 0,02 & 0,85 \\
\hline
\end{tabular}

Correlação de Spearman. $\Delta$ : diferença relativa entre os valores obtidos nas posições supina e ortostática; CR1 a CR5: coeficiente de recuperação calculado do primeiro ao quinto minuto do protocolo de recuperação 


\section{4- Discussão}

Conforme adotado para apresentação dos resultados, a sessão de discussão será subdividida em três tópicos, de acordo com a apresentação dos resultados.

\section{1- Análise cronotrópica antes, durante e após o teste de esforço submáximo}

Os achados primários do presente estudo revelam que os indivíduos que praticam a dança de salão como atividade física regular apresentaram menor $\mathrm{FC}_{\text {basal }}$, maior desempenho durante o teste de esforço submáximo e um maior decremento da FC após o teste de esforço comparativamente ao grupo $\mathrm{C}$.

Esses resultados indicam a possibilidade da prática de dança de salão, mesmo em condições de esforço não controlado, estar associada à adaptações crônicas positivas sobre o sistema cardiovascular. Diversos estudos demonstram que a prática regular de exercícios físicos pode induzir aumentos no grau de modulação parassimpática (PAGKALOS et al., 2008; SLOAN et al., 2009; BILLMAN et al., 2015), bem como redução da atividade simpática sobre o coração (ROVEDA et al., 2003; CARTER e RAY, 2015). Dessa forma, o cronotropismo cardíaco mais lento observado no grupo de praticantes de dança nas três condições avaliadas talvez seja explicado por um maior tônus vagal e/ou menor tônus simpático sobre o coração desses indivíduos. Adicionalmente, é importante destacar a possibilidade de redução da FC por adaptações intrínsecas no nodo sinoatrial (MOLINA et al., 2013; NEGRAO et al., 2013; AZEVEDO et al., 2014).

Outro aspecto importante a ser destacado é a maior tolerância ao esforço observada no grupo PD. Considerando que a aptidão cardiorrespiratória pode ser expressa em equivalentes metabólicos (METs), e que 1 MET corresponde a aproximadamente $1 \mathrm{~km} / \mathrm{h}$ (LEE et al., 2010), é plausível inferir que o maior tempo de teste observado no grupo PD é um forte indicador de maior aptidão cardiorrespiratória nesses indivíduos em comparação ao grupo controle. Além disso, o maior tempo despendido para alcançar a frequência cardíaca alvo $\left(85 \%\right.$ da $\left.\mathrm{FC}_{\max }\right)$ no grupo PD, já é um indicador de menor sobrecarga cardiovascular em condições de esforço submáximo. 
No período de recuperação pós esforço, o grupo PD apresentou um maior decremento absoluto e relativo da frequência cardíaca durante todo o protocolo quando comparado ao grupo C. Em um clássico e elegante estudo com bloqueio farmacológico, Imai et al. (1994), demonstraram que o decremento da FC no primeiro minuto de recuperação é deprimido pela administração de atropina (um antagonista de receptores muscarínicos), e que o decremento na segunda fase da recuperação, a partir do segundo minuto, é influenciada pela administração de atropina e propranolol (um antagonista dos receptores adrenérgicos), demonstrando o papel da reativação vagal no decremento da frequência cardíaca no início da fase de recuperação e da retirada simpática associada a reativação vagal no decremento da frequência cardíaca em períodos subsequentes.

Diante do exposto, o maior decremento da frequência cardíaca observado no grupo PD sugere ajustes autonômicos mais eficientes nessa condição funcional, quando comparados ao grupo $\mathrm{C}$, o que contempla estes indivíduos com o efeito cardioprotetor e antiarrítmico proporcionado pela maior reativação vagal e/ou retirada simpática nessa condição de estresse físico (BILLMAN, 2002; BILLMAN, 2009).

Ainda sobre o período de recuperação, é importante destacar a sutil análise dos coeficientes. Conforme observado na Tabela 2.5, quando o decremento da frequência cardíaca foi ajustado à reserva cronotrópica alcançada durante o teste de esforço $\left[\mathrm{DFC} /,\left(\mathrm{FC}_{\text {pico }}-\right.\right.$ $\left.\left.\mathrm{FC}_{\text {inicial }}\right)\right]$ não foram encontradas diferenças significativas entre os grupos. Estes achados demonstram que, embora os praticantes de dança apresentem um maior decremento da frequência cardíaca, a recuperação da frequência cardíaca foi similar entre os grupos.

O cálculo dos coeficientes fornece uma informação matematicamente mais plausível sobre a recuperação da frequência cardíaca em relação ao DFC, consistindo em uma medida complementar a esse último. Entende-se como recuperação da frequência cardíaca o retorno aos níveis pré-exercício, assim, seu cálculo deve levar em consideração o valor da $\mathrm{FC}_{\text {inicial }} \mathrm{e}$ o da $\mathrm{FC}_{\text {pico, }}$, conforme o coeficiente adotado no presente estudo. Já o decremento, trata-se da diferença absoluta ou relativa entre a $\mathrm{FC}_{\text {pico }}$ e a $\mathrm{FC}$ registrada no período de recuperação (ex.

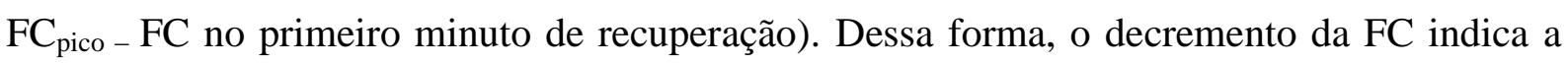
magnitude da recuperação em relação ao pico do esforço e a recuperação da frequência cardíaca o quanto ocorreu de recuperação considerando a reserva cronotrópica.

Com fins ilustrativos, considere um indivíduo (indivíduo 1) que inicia um TES com uma FC de 55 bpm e um segundo (indivíduo 2) que inicia com 85 bpm. Ao final do teste, 
ambos alcançam $165 \mathrm{bpm}$, contudo o ponto de partida do indivíduo 1 é mais baixo. No período de recuperação, o indivíduo 1 apresenta um maior DFC, contudo, a recuperação da frequência cardíaca em relação à reserva cronotrópica é similar (Figura 5)

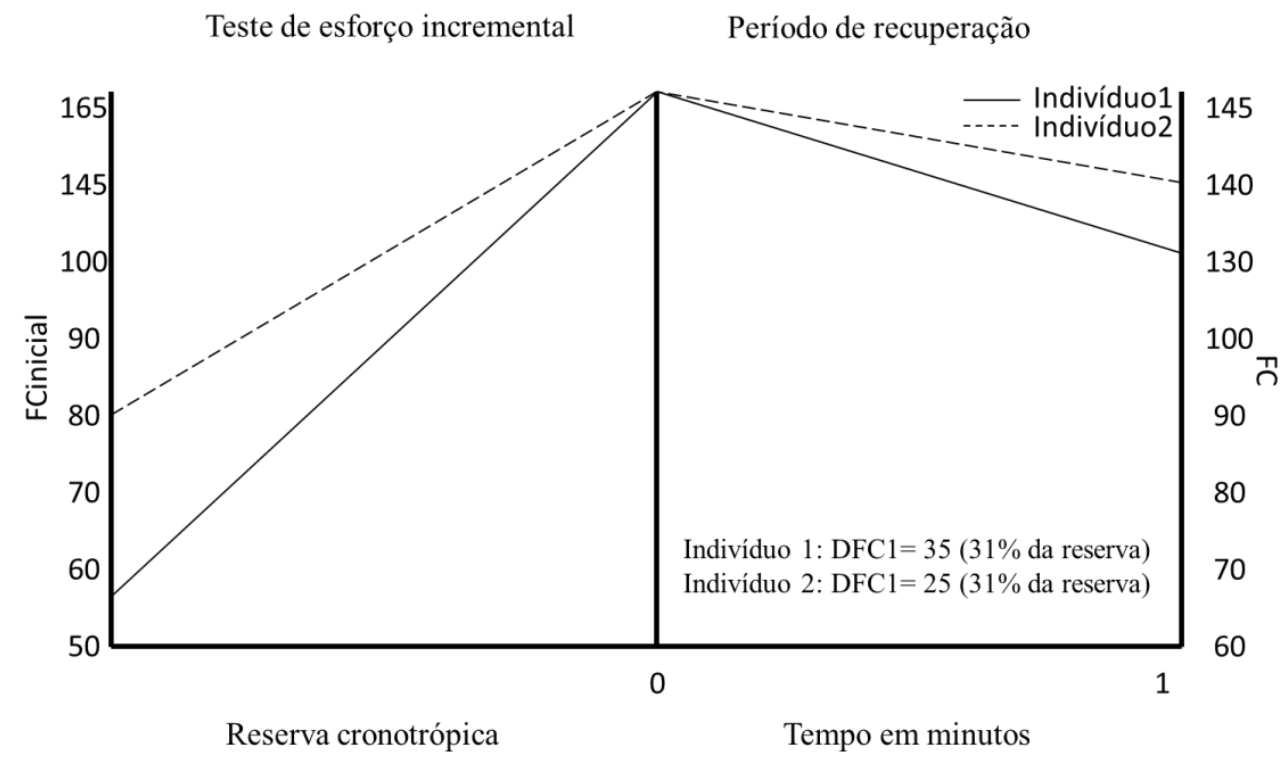

Figura 5- Ilustração da avaliação da recuperação da frequência cardíaca no primeiro minuto pós-exercício considerando a reserva cronotrópica durante o teste de esforço.

Outra análise cronotrópica complementar adotada foi a velocidade do decremento da frequência cardíaca, que permite avaliar o quanto de redução ocorreu no primeiro, segundo, terceiro, quarto, e quinto minuto após o esforço. Em relação é essa variável, a única diferença observada entre os grupos foi uma maior velocidade de decremento da frequência cardíaca no primeiro minuto de recuperação, a qual reflete o decremento da frequência cardíaca nesse período.

Com objetivo de ilustrar esta análise, podemos destacar dados hipotéticos de dois indivíduos que iniciam um TES com valores similares de $\mathrm{FC}_{\text {inicial }}$ e alcançam os mesmos valores de $\mathrm{FC}_{\text {pico, }}$, portanto, possuem reservas cronotrópicas equivalentes. Após 5 minutos de recuperação, os dois também são contemplados com o mesmo decremento total da FC ao final do quinto minuto pós esforço. Entretanto, o indivíduo 1 reduziu $64 \%$ do decremento total no primeiro minuto, enquanto o indivíduo 2 recupera apenas $32 \%$ nesse mesmo intervalo de tempo, o que indica que o primeiro teve uma maior velocidade de decremento na fase inicial do período pós esforço (Figura 6). 
Teste de esforço incremental Período de recuperação

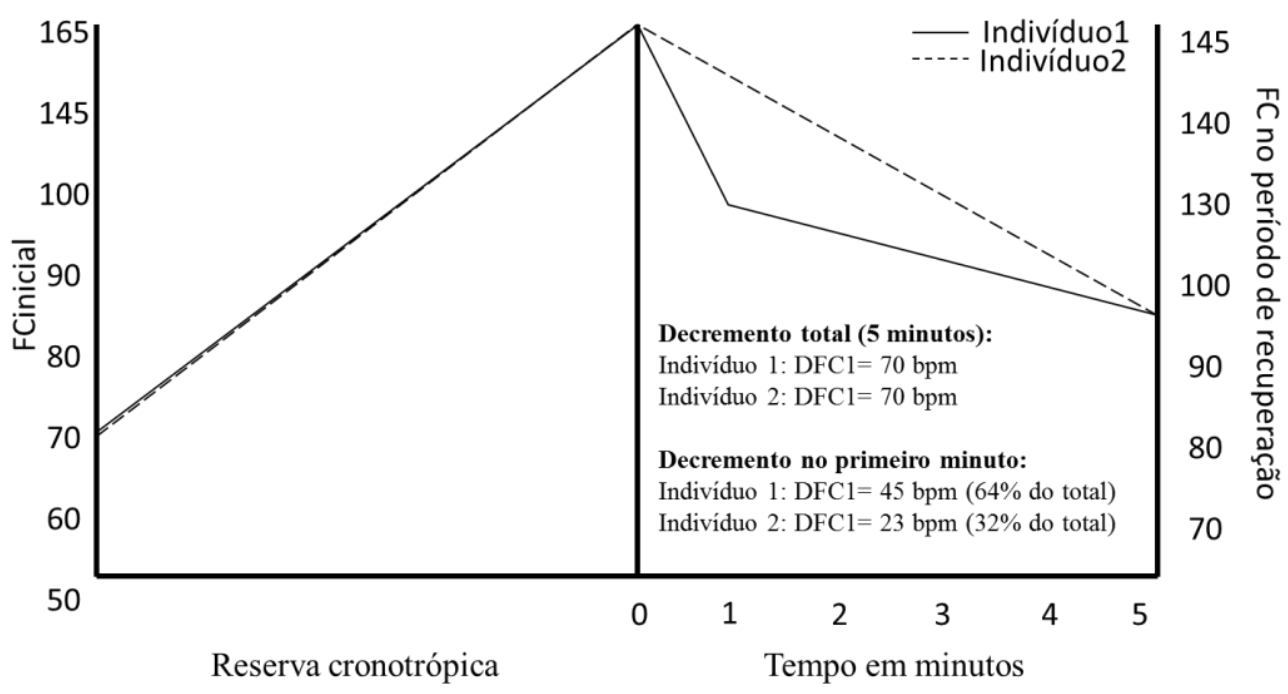

Figura 6- Ilustração da avaliação da velocidade do decremento da frequência cardíaca.

Em resumo, apesar de o grupo PD apresentar um maior decremento da frequência cardíaca em todo o período de recuperação, a recuperação e a velocidade do decremento da FC foram similares entre os grupos em todos os registros. Esses resultados ilustram claramente as valiosas informações complementares proporcionadas pelas análises do CR e da VDFC. Em um primeiro momento, poderíamos inferir erroneamente que o grupo PD foi contemplado com uma maior recuperação da frequência cardíaca, o que na essência da palavra, seria um equívoco.

É importante destacar que as análises do CR e da VD, embora possibilitem uma análise mais abrangente do cronotropismo pós exercício, não refutam o significado fisiológico do DCF, bem como seu valor prognóstico. Tal afirmação é sustentada pelo fato de que este é um marcador de saúde cardiovascular solidamente estabelecido na literatura e que guarda relação com vários desfechos cardiovasculares negativos (FEI et al., 2005; SMITH et al., 2005; SUNG et al., 2006; JAE et al., 2008; JAE et al., 2009; MADDOX et al., 2009; ARENA et al., 2010; YAMADA et al., 2011; ANENI et al., 2014).

De uma forma geral, o comportamento da frequência cardíaca observado nos praticantes de dança nas condições de repouso, esforço e recuperação pós esforço, indicam melhores perspectivas à saúde cardiovascular nesse grupo. Conforme amplamente apresentado na literatura, maiores valores de frequência cardíaca em condição de repouso 
estão associados a um maior risco de desenvolver doenças cardiovasculares e mortalidade por todas as causas (JOUVEN et al., 2001; PIWONSKA et al., 2008; ROGOWSKI et al., 2009; COONEY et al., 2010; FAGUNDES e CASTRO, 2010). Tal poder prognóstico também se estende ao decremento lento da frequência cardíaca no período de recuperação após um teste de esforço (COLE et al., 1999; COLE et al., 2000; NISHIME et al., 2000; VIVEKANANTHAN et al., 2003; SMITH et al., 2005; SUNG et al., 2006; JAE et al., 2008; SAVONEN et al., 2011; YAMADA et al., 2011; CAHALIN et al., 2013) e a baixos níveis de aptidão cardiorrespiratória (BLAIR et al., 1995; CARNETHON et al., 2005; KODAMA et $a l$. , 2009). Por outro lado, é importante destacar que o grupo controle não apresentou comportamento anormal da FC em qualquer condição funcional, embora os valores estejam mais próximos aos valores de referência disponíveis na literatura, quando comparados ao grupo PD.

\section{2- Análise da variabilidade da frequência cardíaca em condição de repouso}

Embora não se tenha observado diferenças consistentes entre os grupos no que se refere a VFC, alguns marcadores fornecem indícios de que o grupo PD seja contemplado com um maior grau de modulação autonômica sobre o coração.

Na posição supina, os maiores valores dos índices Grau, SD2 e área da elipse indicam maiores níveis de modulação global nos praticantes de dança de salão. Ademais, os maiores valores da média dos iRR, SD1, r-MSSD sugerem um estado vagotônico nesses indivíduos.

Na posição ortostática, os maiores valores observados no componente de BFun, em paralelo aos valores mais baixos de AFun, merecem uma análise cautelosa. Inicialmente, poderíamos sugerir que os praticantes de dança possuem característica simpaticotônica e baixa modulação vagal durante o ortostatismo em relação ao grupo C. Entretanto, ao observar a maior variação diante da mudança postural do componente AF no grupo PD, fica claro que o aumento e redução nos índices normalizados de baixa e alta frequência, respectivamente, foram mediados primariamente por uma maior retirada vagal e não pelo aumento da modulação simpática sobre o coração.

Quanto à razão BF/AF na posição ortostática, sua ascensão também pode ser mediada por aumentos no BF, redução no AF ou ambos (SHAFFER et al., 2014). No caso dos 
praticantes de dança, a redução do AF responde por $73 \%$ do aumento na razão quando considerado o valor da mediana, tornando-se incoerente inferir sobre um estado simpaticotônico nesses indivíduos com base nos valores desse marcador. Em contrapartida, a maior retirada vagal, analisada pelo índice AF, sugere que o grupo PD foi contemplado com uma maior capacidade de modulação vagal diante da manobra postural ativa, contudo essa inferência também não é suportada por outros marcadores de modulação vagal.

Em uma análise mais minuciosa descrita previamente por Correia et al. (2007) e apresentada na Tabela 8, pode ser observado que na posição supina, 52\% da amostra do grupo PD possui um ou mais índices temporais acima do terceiro quartil em relação ao grupo C, contra apenas $17 \%$ abaixo do primeiro quartil. Na análise no domínio espectral, esses valores foram de $78 \%$ e $39 \%$, respectivamente.

Em condição de ortostatismo, 47\% dos praticantes de dança apresentaram índices temporais acima do primeiro quartil em relação ao grupo C, contra $39 \%$ abaixo do primeiro quartil. Ainda na posição ortostática, 43\% dos dançarinos apresentaram um ou mais índices espectrais acima do terceiro quartil em relação ao grupo de referência, contra $65 \%$ abaixo do primeiro quartil.

Com exceção da análise espectral na posição ortostática, esses valores demonstram que grande parte dos indivíduos que formam o grupo PD possuem marcadores autonômicos mais elevados em relação ao grupo $\mathrm{C}$, considerando a amplitude interquartílica como referência.

É necessário chamar atenção para o fato de que, apesar das inconsistências relacionadas às diferenças entre os grupos no que se refere à análise da VFC, no grupo PD foram encontrados marcadores autonômicos mais favoráveis em relação ao grupo $\mathrm{C}$, mas o inverso não foi observado em nenhuma análise aqui descrita. De certa forma, não eram esperadas diferenças drásticas na VFC em condição de repouso na comparação entre os grupos, visto que ambos gozam de bom estado de saúde e possuem características antropométricas e idades similares, fatores que sabidamente influenciam a VFC (ANTELMI et al., 2004; ZHANG, 2007; KOENIG et al., 2014). Além disso, o aumento da modulação autonômica cardíaca mediado pela prática de exercícios parece ser mais expressivo em indivíduos com baixos níveis iniciais de modulação (PAGKALOS et al., 2008), o que não é verdade para nossos grupos, que, de acordo com a idade, encontram-se no ápice de suas funções autonômicas (SRINIVASAN et al., 2002). 
Esses achados são acompanhados de relevantes implicações clínicas. Importantes estudos prospectivos sugerem que a redução do grau de modulação autonômica sobre coração pode preceder o desenvolvimento de doenças cardiovasculares (CARNETHON et al., 2003; SCHROEDER et al., 2003), além de estar associada ao aumento no risco de mortalidade em diversas populações (KLEIGER et al., 1987; TSUJI et al., 1994; GERRITSEN et al., 2001; CAMM et al., 2004). Ademais, a atuação parassimpática sobre o coração reduz a susceptibilidade de arritmias malignas, conferindo a este órgão um efeito cardioprotetor (BILLMAN, 2002; NG et al., 2007; NG, 2014). Assim, os maiores valores dos marcadores parassimpáticos (Média de ir, SD1 e r-MSSD) e de modulação global (Grau, SD2 e área da elipse) observados no grupo PD sugerem melhores prognósticos à saúde cardiovascular desses indivíduos.

Outro aspecto importante a ser destacado, é o comportamento da VFC diante da manobra postural ativa. Na transição da posição supina para posição ortostática, a maior variação relativa da área absoluta de alta frequência, área normalizada de alta frequência e da área $<1$, indicam uma retirada vagal mais expressiva no grupo de praticantes de dança diante da alteração postural. Em um estudo prospectivo, Grant et al. (2012), demonstram que o grau de variação autonômica diante da transição supino-ortostático pode ser potencializado pela prática de exercícios físicos, sugerindo que essa intervenção pode aumentar a responsividade do sistema nervoso autônomo diante de um estímulo externo. Essa informação reforça a possível relevância clínica da prática de dança, visto que a redução da responsividade autonômica é uma característica associada ao processo de envelhecimento (LIPSITZ et al., 1990; SRINIVASAN et al., 2002; BARANTKE et al., 2008) e à doenças cardiovasculares (KAMINSKA et al., 2008; MARTINISKOVA et al., 2009; WANG et al., 2012) .

Em síntese, a análise da variabilidade da frequência cardíaca reforça as melhores perspectivas relacionadas à saúde dos praticantes de dança discutidas anteriormente na análise do cronotropismo cardíaco. Contudo, destaca-se que as diferenças encontradas entre os grupos na análise da VFC foram menos expressivas e pouco consistentes. 


\section{3-Análise da variabilidade da frequência cardíaca durante e após o teste de esforço submáximo}

Diante da não estacionariedade dos registros, a análise da variabilidade da frequência cardíaca durante e após o teste de esforço foi realizada por meio dos índices de Poincaré e tempo-frequenciais, marcadores adequados para análise da variabilidade da frequência cardíaca em condições dinâmicas (TULPPO et al., 1996; MAINARDI et al., 2002).

Variabilidade da frequência cardíaca durante o teste de esforço submáximo

Durante o teste de esforço incremental foi observado que o limiar de variabilidade da frequência cardíaca foi alcançado de forma mais tardia no grupo de praticantes de dança. Visto que o SD1 é um indicador válido da modulação parassimpática durante o exercício físico progressivo (TULPPO et al., 1996), é coerente afirmar que nesse grupo a depressão parassimpática ocorreu em intensidades mais elevadas de esforço físico.

Essa observação tem implicações fisiológicas importantes. Do ponto de vista clínico, é amplamente aceito na literatura que a atuação vagal sobre o coração confere a esse órgão um efeito antiarrítmico (BILLMAN, 2002; NG et al., 2007; BILLMAN, 2009), dessa forma, nossos achados sugerem que o grupo de praticantes de dança é contemplado com um maior efeito cardioprotetor em condições de esforço submáximo.

Em uma perspectiva metabólica, várias pesquisas indicam que o ponto de ocorrência do limiar de variabilidade da frequência cardíaca coincide com o limiar anaeróbio (LIMA e KISS, 1999; SALES et al., 2011; MOUROT et al., 2012; LEPRÊTRE et al., 2013), o que oferece indícios de que o grupo de praticantes de dança tenha uma maior capacidade aeróbia em relação ao grupo controle. Entretanto, conforme destacado em uma revisão recente publicada por nosso grupo (GOMES e MOLINA, 2014), a aplicação do limiar de variabilidade da frequência cardíaca, avaliado por meio do SD1, como indicador do primeiro limiar ventilatório ainda não é uma conduta solidamente estabelecida na literatura. 
De forma independente à abordagem metabólica, o maior espectro de modulação parassimpática observado no grupo de praticantes de dança durante o teste de esforço é uma adaptação crônica observada em indivíduos envolvidos com a prática de exercícios aeróbios (MOUROT et al., 2004). Dessa forma, talvez o envolvimento nas aulas de dança de salão, mesmo sem o controle da intensidade durante as sessões, tenha fornecido estímulo suficiente para promover tal adaptação nos indivíduos avaliados no presente estudo, fato que precisa ser confirmado em trabalhos posteriores.

\section{Variabilidade da frequência cardíaca após o teste de esforço submáximo}

Considerando os cinco minutos de recuperação após o teste de esforço submáximo, os maiores valores observados dos índices GRAU, Centróide, SD2 e área da elipse, indicam que o grupo de praticantes de dança apresenta um maior grau de modulação autonômica cardíaca global em relação ao grupo controle nessa condição de estresse. Além disso, foi observada uma tendência $(\mathrm{p}=0,06)$ a maiores valores do índice SD1, um marcador de modulação parassimpática.

Visto que as diferenças encontradas não permitem uma discriminação entre a atuação dos componentes simpático e parassimpático, torna-se limitada a possibilidade de explicação do fenômeno fisiológico predominante nesse período. Contudo, vale ressaltar que os maiores valores de marcadores globais, sejam eles mediados por menor atuação simpática e/ou maior atuação parassimpática, devem ser interpretados como uma característica positiva. Essa afirmação é sustentada em dados disponíveis na literatura que revelam que a hiperatividade simpática e ou a depressão parassimpática são considerados fatores pró-arritmogênicos (BILLMAN, 2002; BILLMAN e KUKIELKA, 2006; NG et al., 2007; NG et al., 2009).

\section{4-Correlação entre a variabilidade da frequência cardíaca de repouso e o decremento absoluto da frequência cardíaca após o teste de esforço}

Com exceção dos indicadores de cronotropismo cardíaco, número de iRR e média dos iRR, não foram observadas correlações importantes e/ou significativas entre a VFC de repouso e o decremento da frequência cardíaca do primeiro ao quarto minuto de recuperação. Por outro lado, alguns marcadores de modulação global e de modulação parassimpática foram 
positivamente correlacionados de forma consistente com o quinto minuto de recuperação após o teste de esforço submáximo.

Diante das inconsistências e/ou dos valores limitados das correlações observadas entre a maioria dos índices de variabilidade da frequência cardíaca em repouso e o decremento da frequência cardíaca, não parece sensato inferir sobre qualquer associação fisiológica entre os dois fenômenos. Contudo, as consistentes correlações negativas observadas entre o número de iRR e número de pontos com o decremento da frequência cardíaca a partir do segundo minuto, bem como as correlações positivas observadas entre a média de iRR e do centróide com o decremento da frequência cardíaca nesse mesmo período indicam que a bradicardia de repouso está associada ao decremento da frequência cardíaca a partir do segundo minuto de recuperação após a realização de um teste de esforço submáximo.

Nos últimos anos, alguns autores buscaram avaliar essa possível correlação com o objetivo de averiguar se indivíduos com maior grau de modulação autonômica na condição de repouso são necessariamente contemplados com um maior decremento da frequência cardíaca nos primeiros minutos de recuperação, o qual é dependente primariamente da atuação neural (IMAI et al., 1994). Nesse cenário investigativo, os resultados são limitados e controversos (JAVORKA et al., 2002; EVRENGUL et al., 2006; ESCO et al., 2010; DANIELI et al., 2014).

Javorka et al. (1994) e Esco et al. (2010), falharam em encontrar correlações estatisticamente significativas entre índices da variabilidade da frequência cardíaca de repouso e o decremento da frequência cardíaca. Por outro lado, em um estudo com coronariopatas, Evrengul et al. (2006), encontraram correlações negativas entre o decremento da frequência cardíaca no terceiro minuto e os índices de baixa frequência $(r=-0,67 \mathrm{p}=$ 0,0001) e a razão BF/AF ( $\mathrm{r}=-0,62 \mathrm{p}=0,0001$, e correlações positivas entre o decremento da frequência cardíaca no terceiro minuto e os índices de alta frequência $(r=0,69 \mathrm{p}=0,0001)$, $\operatorname{SDNN}(r=0,41 \mathrm{p}=0,0001), r-M S S D(r=0,31 \mathrm{p}=0,008)$ e $\mathrm{pNN} 50(\mathrm{r}=0,44 \mathrm{p}=0,0001)$.

Correlações entre a variabilidade da frequência cardíaca de repouso e o decremento da frequência cardíaca também foram observadas no estudo de Danieli et al. (2014). Nessa investigação, o decremento da frequência cardíaca nos primeiros 30, 60 e 120 segundos de recuperação foram correlacionados à potência total, área absoluta de baixa frequência, área absoluta de alta frequência, área normalizada de alta frequência, r-MSSD, razão BF/AF e 
SDNN, ambos obtidos a partir de registros realizados com o voluntário sentado no cicloergômetro.

Diante dos dados previamente disponíveis na literatura, e dos apresentados no presente trabalho, o tema em questão torna-se aparentemente controverso, o que não é necessariamente verdade. As características amostrais e protocolos adotados nos estudos disponíveis são amplamente diversificados, o que impede uma comparação entre os achados obtidos nesses estudos. Dessa forma, o que se tem disponível é uma gama de análises em diferentes situações, e consequentemente, resultados aparentemente "conflitantes".

Quanto à possível influência do comportamento autonômico em condição de repouso e o decremento da frequência cardíaca, algumas considerações fisiológicas são importantes. É relevante destacar que a variabilidade da frequência cardíaca é um indicador da modulação autonômica, e não do tônus autonômico sobre o coração (HEDMAN et al., 1995; BUCHHEIT,PAPELIER, et al., 2007), dessa forma, talvez o decremento da frequência cardíaca seja mais dependente desse último, o que limitaria a associação entre as duas variáveis. Outra observação importante é que, em repouso na posição supina, a variabilidade da frequência cardíaca é primariamente influenciada pela atividade vagal (POMERANZ et al., 1985; HAYANO et al., 1991), já o decremento da frequência cardíaca pode ser parcialmente ou primariamente dependente da retirada simpática (IMAI et al., 1994; BUCHHEIT,LAURSEN, et al., 2007).

A própria diferença matemática entre as variáveis também deve ser considerada. $\mathrm{O}$ decremento da frequência cardíaca reflete a magnitude da variação da frequência cardíaca ao longo de dois pontos em um determinado tempo, enquanto a variabilidade da frequência cardíaca reflete as oscilações temporais entre ciclos cardíacos consecutivos, ou seja, a cada batimento (BUCHHEIT,PAPELIER, et al., 2007).

Destaca-se ainda que o sistema nervoso autônomo tem atuação dinâmica, ou seja, seu arcabouço funcional envolve regulação, aumentando ou reduzindo as atividades simpática e parassimpática sobre um determinado órgão. Nesse sentido, quando se correlaciona a variabilidade da frequência cardíaca de repouso com o decremento da frequência cardíaca, busca-se associação entre duas variáveis que estão sob influencia do sistema nervoso autônomo, entretanto, uma em condição estática e outra em condição dinâmica. 
Nesse sentido, Molina e colaboradores (2013), investigaram a correlação entre a variabilidade da frequência cardíaca em repouso (posição supina e ortostática) e o grau de alteração dessa variável na transição da posição supina para ortostática com os decrementos absoluto e relativo da frequência cardíaca após um teste de esforço máximo. Os resultados dessa pesquisa demonstram que, em indivíduos jovens aparentemente saudáveis, a modulação de repouso e a capacidade de ajuste autonômico frente a mudança postural, avaliada pela diferença relativa entre a variabilidade da frequência cardíaca nas posições supina e ortostática, estão significativamente correlacionadas com os decrementos absoluto e relativo da frequência cardíaca.

Em síntese, a possível associação entre a modulação autonômica basal e seu comportamento diante de condições de estresse não foi solidamente confirmada no presente estudo e ainda necessita de maiores esclarecimentos, visto que as correlações estatisticamente significativas encontradas nos estudos mencionados acima variam de fracas a moderadas $(r=$ 0,30 a 0,60, aproximadamente), o que indica a possibilidade de outras variáveis estarem associadas ao controle do decremento da frequência cardíaca após um teste de esforço.

Assim, visto que tanto a variabilidade da frequência cardíaca quanto o decremento da frequência cardíaca são utilizados para se avaliar a função autonômica cardíaca (BUCHHEIT e GINDRE, 2006; GOLDBERGER et al., 2006; BUCHHEIT,LAURSEN, et al., 2007; BUCHHEIT,PAPELIER, et al., 2007), parece que esses indicadores fornecem informações dissociadas, mas complementares, sobre o status autonômico do avaliado, aspecto já discutido por outros autores (BUCHHEIT,PAPELIER, et al., 2007).

4.5-Correlação entre a variabilidade da frequência cardíaca em repouso e o coeficiente de recuperação da frequência cardíaca

Visto que o decremento da frequência cardíaca e o coeficiente de recuperação da frequência cardíaca mensuram diferentes aspectos relacionados ao cronotropismo cardíaco, foi testada a hipótese de uma possível correlação entre a variabilidade da frequência cardíaca registrada em condição de repouso com a recuperação da frequência cardíaca, indicada pelo coeficiente de recuperação.

Da mesma forma que para o decremento da frequência cardíaca, o coeficiente de recuperação em nenhum momento foi significativamente associado à variabilidade da frequência cardíaca em condição de repouso, indicando que, além do decremento da 
frequência cardíaca as oscilações entre os intervalos R-R em repouso também não estão relacionadas à recuperação da frequência cardíaca.

Visto que tanto o decremento da frequência cardíaca quanto o coeficiente de recuperação consideram a magnitude de variação da frequência cardíaca entre dois pontos em um intervalo de tempo, os aspectos discutidos na sessão anterior se estendem à sessão atual.

\section{Considerações finais}

As expressivas diferenças encontradas entre os grupos no que se refere ao comportamento cronotrópico nas três condições funcionais avaliadas, repouso, esforço e recuperação indicam melhores perspectivas à saúde cardiovascular dos praticantes de dança em relação ao grupo controle.

Adicionalmente, além da frequência cardíaca per se, as análises da variabilidade da frequência cardíaca nas condições de repouso, esforço e recuperação pós esforço, fornecem fortes indícios de que o grupo de praticantes de dança é contemplado com uma maior modulação autonômica sobre o coração, o que confere um efeito protetor a esse órgão.

Por fim, não foram observadas correlações consistentes entre a modulação autonômica em repouso, avaliada por meio da variabilidade da frequência cardíaca, e o comportamento da frequência cardíaca no período de recuperação imediatamente após a realização do teste de esforço submáximo. Esses achados sugerem que as duas variáveis em questão fornecem informações distintas, porém complementares, sobre o status autonômico cardíaco.

\section{Limitações do estudo}

Como limitações do estudo podemos destacar seu caráter transversal e a técnica de amostragem adotada em nosso delineamento. Dessa forma, não é possível destacar uma relação de causa e efeito quanto à prática de dança de salão e os marcadores autonômicos mais promissores observados nos praticantes de dança de salão.

Podemos destacar diversos fatores não controlados no presente estudo que podem estar associados ao melhor perfil cardiovascular observado nos praticantes de dança. Inicialmente podemos mencionar a prática de dança em momentos de lazer, como a 
participação em bailes e/ou aulas práticas oferecidas pelas escolas de dança aos finais de semana, assim a prática de atividade física não seria restrita às aulas de dança propriamente ditas.

Outro possível fator confundidor seria o estado emocional dos indivíduos envolvidos com a prática de dança, ou seja, o próprio caráter lúdico/emocional associado à prática da dança poderia levar a alterações positivas no perfil autonômico dos indivíduos, aspecto já evidenciado na literatura.

Por outro lado, do ponto de vista da saúde pública, o fator causal não refuta a relevância de nossos achados. Ou seja, embora não esteja claro o fator, ou os fatores, determinantes para o melhor perfil cardiovascular observado nos praticantes de dança, estes indivíduos são contemplados com menor risco cardiovascular considerando o grupo controle. Essa característica é de grande importância visto que as doenças cardiovasculares, cujo tratamento envolve um alto custo para os cofres públicos, respondem pela maior causa de morte no mundo.

\section{5-Conclusões}

Nesta pesquisa desenvolvida com homens praticantes de dança de salão comparativamente a homens sedentários e/ou insuficientemente ativos, observou-se que:

1- Os praticantes de dança de salão são caracterizados por cronotropismo cardíaco mais lento em relação ao grupo controle nas condições de repouso, esforço e recuperação após o esforço;

2- Embora não verificado de forma consistente, a análise da função autonômica cardíaca por meio da variabilidade da frequência cardíaca fornece fortes indícios de que o grupo de praticantes de danças seja contemplado com um maior grau de modulação autonômica cardíaca nas condições de repouso, esforço e recuperação após o esforço;

3-Não foram observadas, de forma consistente, correlações estatisticamente significativas entre os diversos índices da variabilidade da frequência cardíaca em condição de repouso e o comportamento da frequência cardíaca após a realização de um teste de esforço submáximo.

4-Por fim, os resultados encontrados sugerem melhores perspectivas à saúde cardiovascular dos praticantes de dança de salão comparativamente ao grupo controle no repouso-esforço e 
após o esforço. Os achados indicam que o envolvimento com a modalidade de dança de salão, ainda que em um cenário recreacional, pode ser importante conduta no contexto da promoção da pratica da atividade física e de saúde pública. No âmbito da fisiologia cardiovascular, clínica e do exercício, os possíveis desdobramentos da atual pesquisa indicam que o comportamento da frequência cardíaca imediatamente após o teste de esforço submáximo, em indivíduos jovens fisicamente ativos, parece não estar na dependência do grau de modulação da função autonômica cardíaca na condição de repouso. Por fim, os achados sinalizam a necessidade de novas pesquisas que considerem outros possíveis mecanismos associados a esse evento fisiológico, por exemplo, o tônus parassimpático na condição de repouso. 


\section{6-Referências bibliográficas}

AIRES, M. M. Fisiologia. $4^{\mathrm{a}}$ ed. Rio de Janeiro: Guanabara Koogan, 2012.

ALMEIDA, M. B.; ARAÚJO, C. G. S. Effects of aerobic training on heart rate. $\underline{\text { Rev }}$ Bras Med Esp, v.9, n.2. 2003.

ANENI, E. et al. Delayed heart rate recovery is strongly associated with early and latestage prehypertension during exercise stress testing. Am J Hyp, v.27, n.4, Apr, p.514-21. 2014.

ANOSOV, O. et al. High-frequency oscillations of the heart rate during ramp load reflect the human anaerobic threshold. Eur J Appl Physiol, v.83, n.4 -5, Nov, p.388-94. 2000.

ANTELMI, I. et al. Influence of age, gender, body mass index, and functional capacity on heart rate variability in a cohort of subjects without heart disease. Am J Cardiol, v.93, n.3, Feb 1, p.381-5. 2004.

ARENA, R. et al. The influence of sex on the relationship between heart rate recovery and other cardiovascular risk factors in apparently healthy subjects. Scand J Med Sci Sports, v.20, n.2, Apr, p.291-7. 2010.

AZEVEDO, L. F. et al. Sport Modality Affects Bradycardia Level and Its Mechanisms of Control in Professional Athletes. Int J Sports Med, Jul 10. 2014.

BALCIOGLU, S. et al. Heart rate variability and heart rate turbulence in patients with type 2 diabetes mellitus with versus without cardiac autonomic neuropathy. Am J Cardiol, v.100, n.5, Sep 1, p.890-3. 2007.

BARANTKE, M. et al. Effects of gender and aging on differential autonomic responses to orthostatic maneuvers. J Cardiovasc Electrophysiol, v.19, n.12, Dec, p.1296-303. 2008.

BAUMERT, M. et al. Short-term heart rate variability and cardiac norepinephrine spillover in patients with depression and panic disorder. Am J Physiol Heart Circ Physiol, v.297, n.2, Aug, p.H674-9. 2009.

BELARDINELLI, R. et al. Waltz dancing in patients with chronic heart failure: new form of exercise training. Circ Heart Fail, v.1, n.2, Jul, p.107-14. 2008. 
BHAGYALAKSHMI, S. et al. Effect of supervised integrated exercise on heart rate variability in type 2 diabetes mellitus. Kardiologia polska, v.65, n.4, Apr, p.363-369. 2007.

BILLMAN, G. E. Aerobic exercise conditioning: a nonpharmacological antiarrhythmic intervention. J Appl Physiol, v.92, n.2, Feb, p.446-54. 2002.

BILLMAN, G. E. Cardiac autonomic neural remodeling and susceptibility to sudden cardiac death: effect of endurance exercise training. Am J Physiol Heart Circ Physiol, v.297, n.4, Oct, p.H1171-93. 2009.

BILLMAN, G. E. et al. Exercise training induced bradycardia: evidence for enhanced parasympathetic regulation without changes in "intrinsic" sinoatrial node function. $\underline{\mathbf{J}}$ Appl Physiol (1985), Mar 6, p.jap 01111 2014. 2015.

BILLMAN, G. E.; KUKIELKA, M. Effects of endurance exercise training on heart rate variability and susceptibility to sudden cardiac death: protection is not due to enhanced cardiac vagal regulation. J Appl Physiol, v.100, n.3, Mar, p.896-906. 2006.

BLAIR, S. N. et al. Changes in physical fitness and all-cause mortality. A prospective study of healthy and unhealthy men. JAMA, v.273, n.14, Apr 12, p.1093-8. 1995.

BOLEA, J. et al. Non-linear HRV indices under autonomic nervous system blockade. Conference proceedings : ... Annual International Conference of the IEEE Engineering in Medicine and Biology Society. IEEE Engineering in Medicine and Biology Society. Annual Conference, v.2014, Aug, p.3252-5. 2014.

BOSKOVIC, A. et al. Prognostic value of heart rate variability in post-infarction patients. Military-medical and pharmaceutical review, v.71, n.10, Oct, p.925-30. 2014.

BROWN, T. E. et al. Important influence of respiration on human R-R interval power spectra is largely ignored. J Appl Physiol (1985), v.75, n.5, Nov, p.2310-7. 1993.

BRUNETTO, A. F. et al. Limiar ventilatório e variabilidade da frequência cardíaca em adolescentes. Rev Bras Med Esporte, v.11, n.1, p.22-27. 2005.

BUCHHEIT, M.; GINDRE, C. Cardiac parasympathetic regulation: respective associations with cardiorespiratory fitness and training load. Am J Physiol Heart Circ Physiol, v.291, n.1, Jul, p.H451-8. 2006.

BUCHHEIT, M. et al. Parasympathetic reactivation after repeated sprint exercise. Am J Physiol Heart Circ Physiol, v.293, n.1, Jul, p.H133-41. 2007. 
BUCHHEIT, M. et al. Noninvasive assessment of cardiac parasympathetic function: postexercise heart rate recovery or heart rate variability? Am J Physiol Heart Circ Physiol, v.293, n.1, Jul, p.H8-10. 2007.

CAHALIN, L. P. et al. Heart rate recovery after the 6 min walk test rather than distance ambulated is a powerful prognostic indicator in heart failure with reduced and preserved ejection fraction: a comparison with cardiopulmonary exercise testing. Eur J Heart Fail, v.15, n.5, May, p.519-27. 2013.

CAMM, A. J. et al. Mortality in patients after a recent myocardial infarction: a randomized, placebo-controlled trial of azimilide using heart rate variability for risk stratification. Circulation, v.109, n.8, Mar 2, p.990-6. 2004.

CARNETHON, M. R. et al. Prospective investigation of autonomic nervous system function and the development of type 2 diabetes: the Atherosclerosis Risk In Communities study, 1987-1998. Circulation, v.107, n.17, May 6, p.2190-5. 2003.

CARNETHON, M. R. et al. Prevalence and cardiovascular disease correlates of low cardiorespiratory fitness in adolescents and adults. JAMA, v.294, n.23, Dec 21, p.2981-8. 2005.

CARNETHON, M. R. et al. Correlates of the shift in heart rate variability with an active postural change in a healthy population sample: The Atherosclerosis Risk In Communities study. Am Heart J, v.143, n.5, May, p.808-13. 2002.

CARTER, J. R.; RAY, C. A. Sympathetic neural adaptations to exercise training in humans. Auton Neurosci, v.188, Mar, p.36-43. 2015.

CARVALHO, J. L. et al. Study of optimal order for the auto-regressive time-frequency analysis of heart rate variability. Proceedings of the 25th Annual International Conference of the IEEE EMBC. [S.I], p17-21., v.3. 2003.

CHAPLEAU, M. W.; SABHARWAL, R. Methods of assessing vagus nerve activity and reflexes. Heart Fail Rev, v.16, n.2, Mar, p.109-27. 2011.

CHEN, J. Y. et al. Cardiac autonomic functions derived from short-term heart rate variability recordings associated with heart rate recovery after treadmill exercise test in young individuals. Heart Vessels, v.26, n.3, May, p.282-8. 2011.

CHRYSOHOOU, C. et al. Cardiovascular effects of high-intensity interval aerobic training combined with strength exercise in patients with chronic heart failure. A randomized phase III clinical trial. Int J Cardiol, v.179, Jan 20, p.269-74. 2015. 
COHEN, J. L. et al. Cardiorespiratory responses to ballet exercise and the VO2max of elite ballet dancers. Med Sci Sports Exerc, v.14, n.3, p.212-7. 1982.

COLE, C. R. et al. Heart-rate recovery immediately after exercise as a predictor of mortality. N Engl J Med, v.341, n.18, Oct 28, p.1351-7. 1999.

COLE, C. R. et al. Heart rate recovery after submaximal exercise testing as a predictor of mortality in a cardiovascularly healthy cohort. Ann Intern Med, v.132, n.7, Apr 4, p.552-5. 2000.

COONEY, M. T. et al. Elevated resting heart rate is an independent risk factor for cardiovascular disease in healthy men and women. Am Heart J, v.159, n.4, Apr, p.612619 e3. 2010.

COOTE, J. H. Recovery of heart rate following intense dynamic exercise. Exp Physiol, v.95, n.3, Mar, p.431-40. 2010.

CRAIG, C. L. et al. International physical activity questionnaire: 12-country reliability and validity. Med Sci Sports Exerc, v.35, n.8, Aug, p.1381-95. 2003.

DANIELI, A. et al. Resting heart rate variability and heart rate recovery after submaximal exercise. Clin Auton Res, v.24, n.2, Apr, p.53-61. 2014.

DANILOWICZ-SZYMANOWICZ, L. et al. Effect of various forms of physical training on the autonomic nervous system activity in patients with acute myocardial infarction. Kardiologia polska, v.71, n.6, p.558-65. 2013.

DE GODOY, M. F. et al. Preoperative nonlinear behavior in heart rate variability predicts morbidity and mortality after coronary artery bypass graft surgery. Int Med J Exp Clin Res, v.15, n.3, Mar, p.CR117-22. 2009.

DHOBLE, A. et al. Cardiopulmonary fitness and heart rate recovery as predictors of mortality in a referral population. Journal of the American Heart Association, v.3, n.2, p.e000559. 2014.

DIMOPOULOS, S. et al. Effects of exercise rehabilitation program on heart rate recovery in patients with chronic heart failure. Eur J Cardiovasc Prev Rehabil, v.13, n.1, Feb, p.67-73. 2006.

DOMENE, P. A.; EASTON, C. Combined triaxial accelerometry and heart rate telemetry for the physiological characterization of Latin dance in non-professional adults. J Dance Med Sci, v.18, n.1, Mar, p.29-36. 2014. 
DOMENE, P. A. et al. Physiological and perceptual responses to Latin partnered social dance. Hum Movement Sci, v.37, Oct, p.32-41. 2014.

ELLER, N. H. Total power and high frequency components of heart rate variability and risk factors for atherosclerosis. Auton Neurosci, v.131, n.1-2, Jan 30, p.123-30. 2007.

ESCO, M. R. et al. The relationship between resting heart rate variability and heart rate

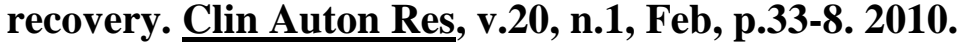

EVRENGUL, H. et al. The relationship between heart rate recovery and heart rate variability in coronary artery disease. Ann Noninvasive Electrocardiol, v.11, n.2, Apr, p.154-62. 2006.

FAGUNDES, J. E.; CASTRO, I. Predictive value of resting heart rate for cardiovascular and all-cause mortality. Arq Bras Cardiol, v.95, n.6, Dec, p.713-9. 2010.

FARRELL, S. W. et al. Cardiorespiratory fitness, body mass index, and heart failure mortality in men: Cooper Center Longitudinal Study. Circ Heart Fail, v.6, n.5, Sep 1, p.898-905. 2013.

FEI, D. Y. et al. Relationship between arterial stiffness and heart rate recovery in apparently healthy adults. Vasc Health Risk Manag, v.1, n.1, p.85-9. 2005.

GAYDA, M. et al. Heart rate recovery after exercise and long-term prognosis in patients

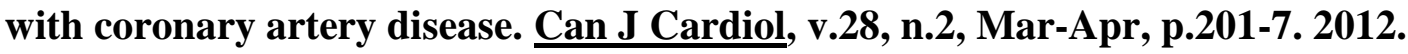

GERRITSEN, J. et al. Impaired autonomic function is associated with increased mortality, especially in subjects with diabetes, hypertension, or a history of cardiovascular disease: the Hoorn Study. Diabetes care, v.24, n.10, Oct, p.1793-8. 2001.

GIELEN, S. et al. Exercise training in patients with heart disease: review of beneficial effects and clinical recommendations. Prog Cardiovasc Dis, v.57, n.4, Jan-Feb, p.347-55. 2015.

GOLDBERGER, J. J. et al. Assessment of parasympathetic reactivation after exercise. Am J Physiol Heart Circ Physiol, v.290, n.6, Jun, p.H2446-52. 2006.

GOLDSTEIN, D. S. et al. Low-frequency power of heart rate variability is not a measure of cardiac sympathetic tone but may be a measure of modulation of cardiac autonomic outflows by baroreflexes. Exp Physiol, v.96, n.12, Dec, p.1255-61. 2011. 
GOMES, C. J.; MOLINA, G. E. Utilização da variabilidade da frequência cardíaca para a identificação do limiar anaeróbio. Uma revisão sistemática. Revista Ed Física, v.25, n.4, p.675-683. 2014.

GOULOPOULOU, S. et al. Exercise training improves cardiovascular autonomic modulation in response to glucose ingestion in obese adults with and without type 2 diabetes mellitus. Metabolism, v.59, n.6, Jun, p.901-910. 2010.

GRANT, C. C. et al. Heart rate variability assessment of the effect of physical training on autonomic cardiac control. Ann Noninvasive Eletrocardiol, v.17, n.3, Jul, p.219-29. 2012.

GUERRA, Z. F. et al. Effects of load and type of physical training on resting and postexercise cardiac autonomic control. Clin Physiol Funct Imaging, v.34, n.2, Mar, p.114-20. 2014.

HA, D. et al. Association of impaired heart rate recovery with cardiopulmonary complications after lung cancer resection surgery. J Thoracic Cardiovasc Surg, Nov 21. 2014.

HABEK, J. C. et al. Left ventricular diastolic function in diabetes mellitus type 2 patients: correlation with heart rate and its variability. Acta Diabetol, v.51, n.6, Dec, p.999-1005. 2014.

HACKNEY, M. E.; EARHART, G. M. Effects of dance on movement control in Parkinson's disease: a comparison of Argentine tango and American ballroom. $\underline{J}$ Rehabil Med, v.41, n.6, May, p.475-81. 2009.

HARRIS, P. R. et al. Heart rate variability measured early in patients with evolving acute coronary syndrome and 1-year outcomes of rehospitalization and mortality. Vasc Health Risk Manag, v.10, p.451-64. 2014.

HAYANO, J. et al. Accuracy of assessment of cardiac vagal tone by heart rate variability in normal subjects. Am J Cardiol, v.67, n.2, Jan 15, p.199-204. 1991.

HAYKOWSKY, M. J. et al. Meta-analysis of aerobic interval training on exercise capacity and systolic function in patients with heart failure and reduced ejection fractions. Am J CArdiol, v.111, n.10, May 15, p.1466-9. 2013.

HEDMAN, A. E. et al. The high frequency component of heart rate variability reflects cardiac parasympathetic modulation rather than parasympathetic 'tone'. Acta Physiol Scand, v.155, n.3, Nov, p.267-73. 1995. 
IELLAMO, F. et al. Effects of a residential exercise training on baroreflex sensitivity and heart rate variability in patients with coronary artery disease: A randomized, controlled study. $\underline{\text { Circulation, }}$, v.102, n.21, Nov 21, p.2588-92. 2000.

IMAI, K. et al. Vagally mediated heart rate recovery after exercise is accelerated in athletes but blunted in patients with chronic heart failure. J Am Coll Cardiol, v.24, n.6, Nov 15, p.1529-35. 1994.

IRIGOYEN, M. C. et al. Controle cardiovascular: regulação reflexa e papel do sistema nervoso simpático. Rev Bras Hipertens, v.8, n.1, p.55-62. 2001.

JAE, S. Y. et al. Slow heart rate recovery after exercise is associated with carotid atherosclerosis. Atherosclerosis, v.196, n.1, Jan, p.256-61. 2008.

JAE, S. Y. et al. Heart rate recovery after exercise and incidence of type 2 diabetes in men. Clin Auton Res, v.19, n.3, Jun, p.189-92. 2009.

JARCZOK, M. N. et al. Lower heart rate variability predicts increased level of Creactive protein 4 years later in healthy, nonsmoking adults. J Int Medicine, v.276, n.6, Dec, p.667-71. 2014.

JAVORKA, M. et al. Heart rate recovery after exercise: relations to heart rate variability and complexity. Braz J Med Biol Res, v.35, n.8, Aug, p.991-1000. 2002.

JOUVEN, X. et al. Resting heart rate as a predictive risk factor for sudden death in middle-aged men. Cardiovasc Res, v.50, n.2, May, p.373-8. 2001.

JUNQUEIRA JR, L. F. Sobre o possível papel da disfunção autonômica cardíaca na morte súbita associada à doença de chagas. Arq Bras Cardiol, v.56, n.6, p.451-456. 1990.

JUNQUEIRA JR, L. F. Insights into the clinical and functional significance of cardiac

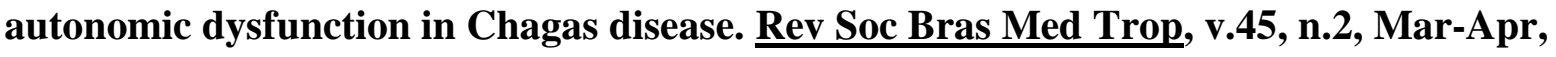
p.243-52. 2012.

KALTSATOU, A. C. et al. Functional and psychosocial effects of either a traditional dancing or a formal exercising training program in patients with chronic heart failure: a comparative randomized controlled study. Clin Rehabil, Jul 17. 2013.

KAMINSKA, A. et al. Spontaneous baroreflex sensitivity in subjects with type 1 diabetes with and without cardiovascular autonomic neuropathy. Endokrynologia Polska, v.59, n.5, Sep-Oct, p.398-402. 2008. 
KANALEY, J. A. et al. Plasticity of heart rate signalling and complexity with exercise training in obese individuals with and without type 2 diabetes. Int J Obesity, v.33, n.10, Oct, p.1198-1206. 2009.

KANNANKERIL, P. J. et al. Parasympathetic effects on heart rate recovery after exercise. J Investig Med, v.52, n.6, Sep, p.394-401. 2004.

KARAPETIAN, G. K. et al. Effect of caffeine on LT, VT and HRVT. Int J Sports Med, v.33, n.7, Jul, p.507-13. 2012.

KARAPETIAN, G. K. et al. Use of heart rate variability to estimate LT and VT. Int J Sports Med, v.29, n.8, Aug, p.652-7. 2008.

KEYHANI, D. et al. Autonomic function change following a supervised exercise program in patients with congestive heart failure. ARYA atherosclerosis, v.9, n.2, Mar, p.150-6. 2013.

KHAN, H. et al. Cardiorespiratory fitness and risk of heart failure: a population-based follow-up study. Eur J Heart Fail, v.16, n.2, Feb, p.180-8. 2014.

KLEIGER, R. E. et al. Decreased heart rate variability and its association with increased mortality after acute myocardial infarction. Am J Cardiol, v.59, n.4, Feb 1, p.256-62. 1987 .

KODAMA, S. et al. Cardiorespiratory fitness as a quantitative predictor of all-cause mortality and cardiovascular events in healthy men and women: a meta-analysis. JAMA, v.301, n.19, May 20, p.2024-35. 2009.

KOENIG, J. et al. Body mass index is related to autonomic nervous system activity as measured by heart rate variability--a replication using short term measurements. $\underline{\mathrm{J} \text { Nutr}}$ Health Aging, v.18, n.3, Mar, p.300-2. 2014.

LACASSE, M. et al. Post-exercise heart rate recovery and mortality in chronic obstructive pulmonary disease. Respir Med, v.99, n.7, Jul, p.877-86. 2005.

LEE, D. C. et al. Mortality trends in the general population: the importance of cardiorespiratory fitness. J Psychopharmacol, v.24, n.4 Suppl, Nov, p.27-35. 2010.

LEE, I. M. et al. Effect of physical inactivity on major non-communicable diseases worldwide: an analysis of burden of disease and life expectancy. Lancet, v.380, n.9838, Jul 21, p.219-29. 2012. 
LEPRÊTRE, P. et al. Determination of ventilatory threshold using heart rate variability in patients with heart failure Surgery, v.S12, p.2-6. 2013.

LIMA, J. R. P.; KISS, M. A. P. D. M. Limiar de variabilidade da frequência cardíaca. Ver Bras Ativ Fis Saúde, v.4, n.1, p.29-38. 1999.

LIPSITZ, L. A. et al. Spectral characteristics of heart rate variability before and during postural tilt. Relations to aging and risk of syncope. Circulation, v.81, n.6, Jun, p.180310. 1990.

LOIMAALA, A. et al. Exercise training improves baroreflex sensitivity in type 2 diabetes. Diabetes, v.52, n.7, Jul, p.1837-1842. 2003.

MADDOX, T. M. et al. Impaired heart rate recovery is associated with new-onset atrial fibrillation: a prospective cohort study. BMC cardiovascular disorders, v.9, p.11. 2009.

MAINARDI, L. T. et al. Time-frequency and time-varying analysis for assessing the dynamic responses of cardiovascular control. Crit Rev Biomed Eng, v.30, n.1-3, p.175217. 2002.

MARTINISKOVA, Z. et al. Baroreflex sensitivity in patients with type I diabetes mellitus. Neuro endocrinology letters, v.30, n.4, p.491-5. 2009.

MELILLO, P. et al. Heart rate variability and target organ damage in hypertensive patients. BMC cardiovascular disorders, v.12, p.105. 2012.

METZLER, M. et al. Neurogenic orthostatic hypotension: pathophysiology, evaluation, and management. J Neurol, v.260, n.9, Sep, p.2212-9. 2013.

MOAK, J. P. et al. Supine low-frequency power of heart rate variability reflects baroreflex function, not cardiac sympathetic innervation. Cleve Clin J Med, v.76 Suppl 2, Apr, p.S51-9. 2009.

MOLINA, G. E. et al. Unaltered R-R interval variability and bradycardia in cyclists as compared with non-athletes. Clin Auton Res, v.23, n.3, Jun, p.141-8. 2013.

MOTAHARI-TABARI, N. et al. The effect of 8 weeks aerobic exercise on insulin resistance in type 2 diabetes: a randomized clinical trial. Global J Health Sci, v.7, n.1, p.34118. 2015. 
MOUROT, L. et al. Quantitative Poincare plot analysis of heart rate variability: effect of endurance training. Euro J Appl Physiol, v.91, n.1, Jan, p.79-87. 2004.

MOUROT, L. et al. Heart rate variability to assess ventilatory thresholds: reliable in cardiac disease? Eur J Prev Cardiol, v.19, n.6, Dec, p.1272-80. 2012.

NAKAMURA, M. et al. Effects of regular aerobic exercise on post-exercise vagal reactivation in young female. Euro J Sports Sci, v.13, n.6, p.674-80. 2013.

NEGISHI, K. et al. Relation of heart-rate recovery to new onset heart failure and atrial fibrillation in patients with diabetes mellitus and preserved ejection fraction. Am J Cardiol, v.111, n.5, Mar 1, p.748-53. 2013.

NEGRÃO, C. E.; BARRETTO, A. C. P. Cardiologia do exercício: Do atleta ao cardiopata. $3^{\text {a }}$ ed São Paulo: Manole, 2010

NEGRAO, C. E. et al. Commentaries on Viewpoint: Is the resting bradycardia in athletes the result of remodeling of the sinoatrial node rather than high vagal tone? $\underline{\mathbf{J}}$ Appl Physiol, v.114, n.9, May, p.1356-7. 2013.

NEGRAO, C. E. et al. Effects of exercise training on neurovascular control and skeletal myopathy in systolic heart failure. Am J Physiol Heart Circ Physiol, v.308, n.8, Apr 15, p.H792-H802. 2015.

NG, G. A. Vagal modulation of cardiac ventricular arrhythmia. Exp Physiol, v.99, n.2, Feb, p.295-9. 2014.

NG, G. A. et al. Autonomic modulation of electrical restitution, alternans and ventricular fibrillation initiation in the isolated heart. Cardiovasc Res, v.73, n.4, Mar 1, p.750-60. 2007.

NG, J. et al. Autonomic effects on the spectral analysis of heart rate variability after exercise. Am J Physiol Heart Circ Physiol, v.297, n.4, Oct, p.H1421-8. 2009.

NISHIME, E. O. et al. Heart rate recovery and treadmill exercise score as predictors of mortality in patients referred for exercise ECG. JAMA, v.284, n.11, Sep 20, p.1392-8. 2000.

PAGKALOS, M. et al. Heart rate variability modifications following exercise training in type 2 diabetic patients with definite cardiac autonomic neuropathy. Br J Sports Med, v.42, n.1, Jan, p.47-54. 2008. 
PANZER, C. et al. Association of fasting plasma glucose with heart rate recovery in healthy adults: a population-based study. Diabetes, v.51, n.3, Mar, p.803-7. 2002.

PIERPONT, G. L.; VOTH, E. J. Assessing autonomic function by analysis of heart rate recovery from exercise in healthy subjects. Am J Cardiol, v.94, n.1, Jul 1, p.64-8. 2004.

PIWONSKA, A. et al. The relationship between resting heart rate and atherosclerosis risk factors. Kardiol Pol, v.66, n.10, Oct, p.1069-75; discussion 1076-8. 2008.

POLANCZYK, C. A. et al. Sympathetic nervous system representation in time and frequency domain indices of heart rate variability. Eur J Appl Physiol Occup Physiol, v.79, n.1, Dec, p.69-73. 1998.

POMERANZ, B. et al. Assessment of autonomic function in humans by heart rate spectral analysis. Am J Physiol, v.248, n.1 Pt 2, Jan, p.H151-3. 1985.

PORTO, L. G.; JUNQUEIRA, L. F., JR. Comparison of time-domain short-term heart interval variability analysis using a wrist-worn heart rate monitor and the conventional electrocardiogram. Pacing Clin Electrophysiol, v.32, n.1, Jan, p.43-51. 2009.

RAMOS, R. P. et al. Heart rate recovery in pulmonary arterial hypertension: relationship with exercise capacity and prognosis. Am Heart J, v.163, n.4, Apr, p.580-8. 2012.

ROGOWSKI, O. et al. Elevated resting heart rate is associated with the metabolic syndrome. Cardiovasc Diabetol, v.8, p.55. 2009.

ROVEDA, F. et al. The effects of exercise training on sympathetic neural activation in advanced heart failure: a randomized controlled trial. J Am Coll Cardiol, v.42, n.5, Sep 3, p.854-60. 2003.

RYAN, M. L. et al. Heart rate variability is an independent predictor of morbidity and

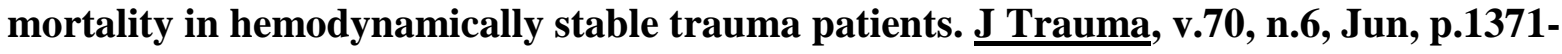
80. 2011.

SALES, M. M. et al. Noninvasive method to estimate anaerobic threshold in individuals with type 2 diabetes. Diabetol Metab Syndr, v.3, n.1, p.1. 2011.

SAVIN, W. M. et al. Autonomic contribution to heart rate recovery from exercise in humans. J Appl Physiol, v.53, n.6, Dec, p.1572-5. 1982. 
SAVONEN, K. P. et al. Two-minute heart rate recovery after cycle ergometer exercise and all-cause mortality in middle-aged men. J Intern Med, v.270, n.6, Dec, p.589-96. 2011.

SAWADA, S. S. et al. Cardiorespiratory fitness, body mass index, and cancer mortality: a cohort study of Japanese men. BMC public health, v.14, p.1012. 2014.

SCHNEIDER, D. A. et al. Plasma catecholamine and blood lactate responses to incremental arm and leg exercise. Med Sci Sports Med, v.32, n.3, Mar, p.608-13. 2000.

SCHROEDER, E. B. et al. Hypertension, blood pressure, and heart rate variability: the Atherosclerosis Risk in Communities (ARIC) study. Hypertension, v.42, n.6, Dec, p.1106-11. 2003.

SHAFFER, F. et al. A healthy heart is not a metronome: an integrative review of the heart's anatomy and heart rate variability. Front Psycol, v.5, p.1040. 2014.

SLOAN, R. P. et al. The effect of aerobic training and cardiac autonomic regulation in young adults. Am J Public Health, v.99, n.5, May, p.921-8. 2009.

SMITH, A. L. et al. Correlated Poincare indices for measuring heart rate variability. Australasian physical \& engineering sciences in medicine, v.30, n.4, Dec, p.336-41. 2007.

SMITH, L. L. et al. Heart rate recovery after exercise: a predictor of ventricular fibrillation susceptibility after myocardial infarction. Am J Physiol Heart Circ Physiol, v.288, n.4, Apr, p.H1763-9. 2005.

SOCIEDADE BRASILEIRA DE CARDIOLOGIA. VI Diretrizes brasileiras de hipertensão arterial. Rev Bras Hipertens, v.17, n.1, Jan-Mar. 2010

SRIDHAR, B. et al. Increase in the heart rate variability with deep breathing in diabetic patients after 12-month exercise training. Tohoku J Exp Med, v.220, n.2, p.107-13. 2010.

SRINIVASAN, K. et al. Effect of standing on short term heart rate variability across age. Clin Physiol Funct Imaging, v.22, n.6, Nov, p.404-8. 2002.

SUNG, J. et al. Metabolic syndrome is associated with delayed heart rate recovery after exercise. J Korean Med Sci, v.21, n.4, Aug, p.621-6. 2006.

SUZUKI, M. et al. Nonlinear measures of heart rate variability and mortality risk in hemodialysis patients. Clin J Am Soc Nephrol, v.7, n.9, Sep, p.1454-60. 2012. 
TAK, Y. J. et al. 25-hydroxyvitamin D and its relationship with autonomic dysfunction using time- and frequency-domain parameters of heart rate variability in Korean populations: a cross-sectional study. Nutrients, v.6, n.10, Oct, p.4373-88. 2014.

TANAKA, H. et al. Age-predicted maximal heart rate revisited. J Am Coll Cardiol, v.37, n.1, Jan, p.153-6. 2001.

\section{TASK FORCE OF THE EUROPEAN SOCIETY OF CARDIOLOGY AND THE} NORTH AMERICAN SOCIETY OF PACING AND ELECTROPHYSIOLOGY. Heart rate variability: Standards of measurement, physiological interpretation, and clinical use. Eur Heart J, v. 17, n. 3, mar, p. 354-81. 1996

THOMAS, G. D. Neural control of the circulation. Adv Physiol Educ, v.35, n.1, Mar, p.28-32. 2011.

TRUCCOLO, A. B. et al. Association between endothelial function and autonomic modulation in patients with Chagas disease. Arq Bras Cardiol, v.100, n.2, Feb, p.135-40. 2013.

TSUJI, H. et al. Reduced heart rate variability and mortality risk in an elderly cohort. The Framingham Heart Study. Circulation, v.90, n.2, Aug, p.878-83. 1994.

TUKEK, T. et al. Effect of diurnal variability of heart rate on development of arrhythmia in patients with chronic obstructive pulmonary disease. Int J Cardiol, v.88, n.2-3, Apr, p.199-206. 2003.

TULPPO, M. P. et al. Quantitative beat-to-beat analysis of heart rate dynamics during exercise. Am J Physiol, v.271, n.1 Pt 2, Jul, p.H244-52. 1996.

VANDERLEI, L. C. M. et al. Noções básicas de variabilidade da frequência cardíaca e sua aplicabilidade clínica. Rev Bras Cir Cardiovasc, v.24, n.2, p.205-217. 2009.

VIVEKANANTHAN, D. P. et al. Heart rate recovery after exercise is a predictor of mortality, independent of the angiographic severity of coronary disease. J Am Coll Cardiol, v.42, n.5, Sep 3, p.831-8. 2003.

WANG, S. et al. Blood pressure regulation in diabetic patients with and without peripheral neuropathy. Am J Physiol, v.302, n.5, Mar 1, p.R541-50. 2012.

WYON, M. A. et al. Oxygen uptake during modern dance class, rehearsal, and performance. J Strength Cond Res, v.18, n.3, Aug, p.646-9. 2004. 
YAMADA, T. et al. Heart rate recovery after exercise is a predictor of silent myocardial ischemia in patients with type 2 diabetes. Diabetes care, v.34, n.3, Mar, p.724-6. 2011.

ZHANG, J. Effect of age and sex on heart rate variability in healthy subjects. $\underline{\mathbf{J}}$ Manipulative Physiol Ther, v.30, n.5, Jun, p.374-9. 2007. 


\title{
ANEXOS
}

ANEXO I: Termo de consentimento livre e esclarecido

Projeto de Pesquisa

\section{FUNÇÃO AUTONÔMICA CARDÍACA E DECREMENTO DA FREQUÊNCIA CARDÍACA APÓS TESTE DE ESFORÇO SUBMÁXIMO EM HOMENS PRATICANTES DE DANÇA DE SALÃO}

\author{
Pesquisador \\ Prof. Esp. Carlos Janssen Gomes \\ Aluno do programa de Pós-graduação (mestrado) em ciências Médicas-UnB
}

\section{Orientador}

Prof. Dr. Luiz Fernando Junqueira Jr.

Prof. Titular do Dep. de Clínica Médica - Cardiologia - Faculdade de Medicina - Universidade de Brasília - UnB

\section{Local de Realização}

Laboratório de Avaliação do Desempenho Físico e Saúde, no Centro Universitário UNIEURO.

\section{TERMO DE CONSENTIMENTO LIVRE ESCLARECIDO}

$\mathrm{Eu}$, abaixo assinado, juntamente com os pesquisadores declaro ter lido ou ouvido, e compreendido totalmente o presente termo de meu consentimento para a participação como convidado que fui pelo pesquisador acima indicado da pesquisa, o qual estabelece o seguinte

1. Estou participando de minha livre e espontânea vontade, a convite dos pesquisadores envolvidos, na pesquisa para verificar os mecanismos que regulam a função cardíaca durante o repouso e após teste de esforço com o objetivo de comparar os achados entre diferentes populações.

2. Nenhum tipo de pagamento será realizado devido a minha participação como voluntário nessa pesquisa. Os pesquisadores responsáveis não têm qualquer responsabilidade sobre eventuais problemas pessoais e/ou profissionais de qualquer tipo. Salvo, eventuais problemas médicos e/ou fisiológicos decorrentes diretamente e exclusivamente de minha participação como voluntário nesta pesquisa. 
3. O protocolo geral da pesquisa prevê apenas uma visita ao laboratório. Inicialmente eu serei atendido pelo pesquisador a fim de proceder a uma anamnese (entrevista sobre características e hábitos pessoais), medida de dados antropométricos (peso, altura, composição corporal, frequência cardíaca de repouso), eletrocardiograma de repouso e teste de avaliação da função autonômica cardíaca. Será utilizado um frequencímetro para registro da contagem do número de batimentos do coração (frequiência cardíaca), na posição deitada, em cama de exame médico, e na posição de pé ao lado da cama. Em cada uma das situações de teste, a contagem dos batimentos do coração terá a duração de 5 minutos, com intervalo de alguns minutos entre uma situação e outra. Após estes procedimentos, serei encaminhado à esteira rolante onde serei submetido a um teste de esforço submáximo o qual será interrompido quando for atingida $85 \%$ da FC máx prevista para idade ou pedido para interromper o teste. Logo ao término do teste de esforço irei ficar $5 \mathrm{~min}$ caminhando na esteira .

4. No caso de serem detectadas quaisquer indicações de suspeita de alterações cardiovasculares no comportamento da frequência cardíaca e da pressão arterial o voluntário será comunicado, indicando-se a sugestão de avaliação médica posterior, independente da pesquisa, para investigação.

5. Quando estiver concluída a pesquisa, poderei, sob minha expressa solicitação, ser informado(a) detalhadamente sobre os resultados e ter uma cópia da mesma.

6. Entendo que poderei não ter benefício pela participação nessa pesquisa, a não ser o da consulta médica e da realização de exames especializados que fornecerão informações sobre meu estado de saúde.

7. Os pesquisadores garantem que qualquer informação pessoal será mantida em sigilo, e a descrição da mesma na apresentação da tese de doutorado e nas publicações científicas que decorrerão deste trabalho não identificará a pessoa examinada

8. Tenho assegurado o direito de abandonar a participação nessa pesquisa a qualquer momento, sem qualquer consequência ou prejuízo para mim, bastando para isso comunicar o desejo aos pesquisadores.

Brasília,................de .......................................de ......

Nome.

Assinatura.

Voluntário

Carlos Janssen Gomes

Pesquisador responsável pela coleta de dados 
ANEXO II: Parecer do comitê de ética

จ UNIEURO

\section{CENTRO UNIVERSITÁRIO UNIEURO \\ COMITÊ DE ÉTICA EM PESQUISA}

PARECER N ${ }^{0} 152 / 2011$

Protocolo No: 152B/2011

Projeto: Modulação autonômica cardíaca e recuperação da frequência cardíaca em homens praticantes de dança de salão

Pesquisador Responsável: Guilherme Eckhardt Molina

Instituição Pesquisada::Unieuro

Validade do Parecer: 1 ano

O Comitê de Ética em Pesquisa do Centro Universitário UNIEURO, após apreciação ética do presente projeto, manifesta-se pela APROVAÇÃo do mesmo. Para a emissão do parecer, observou-se as disposições contidas na Resolução 196/96 CNS/MS, que dispõe sobre as diretrizes e normas regulamentadoras em pesquisa envolvendo seres humanos, assim como as suas resoluções complementares. Ressaltamos que o pesquisador deverá observar as responsabilidades que Ihe são atribuídas na Resolução 196/96 CNS/MS, inciso IX.1 e IX.2, em relação ao desenvolvimento do projeto.

Brasília, 22 de novembro de 2011

Atenciosamente,

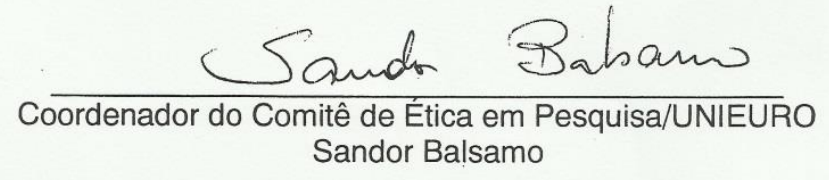

Centro Universitário UNIEURO

www.unieuro.com.br

Av. das Nações, Trecho 0, Conjunto 5, Brasília-DF CEP:70200-000

Tel: (61) 3445-5873 


\section{UNIEURO CENTRO UNIVERSITÁRIO CENTRO UNIVERSITARIO UNIEURO/DF}

\section{PARECER CONSUBSTANCIADO DO CEP}

\section{DADOS DO PROJETO DE PESQUISA}

Título da Pesquisa: MODULAÇÃO AUTONÔMICA CARDÍACA E RECUPERAÇÃO DA FREQUÊNCIA CARDÍACA EM PRATICANTES DE DANÇA DE SALÃO

Pesquisador: CARLOS JANSSEN GOMES

Área Temática:

Versão: 1

CAAE: 35039014.0 .0000 .5056

Instituição Proponente: Centro Universitário UNIEURO/DF

Patrocinador Principal: Financiamento Próprio

\section{DADOS DO PARECER}

Número do Parecer: 791.728

Data da Relatoria: $26 / 08 / 2014$

Apresentação do Projeto:

DE ACORDO, VISTO QUE APRESENTA OS ELEMENTOS FUNDAMENTAIS PARA ELABORAÇÃO DO PROJETO DE PESQUISA AO RIGOR DA METODOLOGIA CIENTÍFICA.

Objetivo da Pesquisa:

COMPATÍVEL COM A INTRODUÇÃO, HIPÓTESE E MÉTODOS

Avaliação dos Riscos e Benefícios:

NÃO FORAM OBSERVADOS RISCOS SIGNIFICATIVOS PARA A AMOSTRA NO PROTOCOLO DO EXPERIMENTO.

Comentários e Considerações sobre a Pesquisa:

DE GRANDE IMPORTÂNCIA E MUITO BEM DELINEADO, METODOLOGICAMENTE.

Considerações sobre os Termos de apresentação obrigatória:

CLAROS, COESOS E COMPATIVEEIS COM AS NECESSIDADES DO PROJETO E O CONSENTIMENTO DA AMOSTRA.

Recomendações:

CERTIFICAR QUE O IPAQ DO GRUPO CONTROLE É DIFERENTE DOS OUTROS GRUPOS.

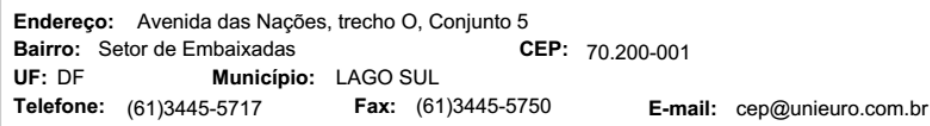


ANEXO IV: Análise de correlação entre a variabilidade da frequência cardíaca em repouso e o decremento relativo da frequência cardíaca

Anexo IV-a:Correlação $(n=46)$ entre os diversos índices temporais das séries de intervalo RR na posição supina previamente ao esforço com o decremento relativo das frequências cardíacas obtidas no período de recuperação ativa após o esforço

\begin{tabular}{|c|c|c|c|c|c|c|c|c|c|c|}
\hline & \multicolumn{2}{|c|}{$\mathrm{DFC}_{1}(\%)$} & \multicolumn{2}{|c|}{$\mathrm{DFC}_{2}(\%)$} & \multicolumn{2}{|c|}{$\mathrm{DFC}_{3}(\%)$} & \multicolumn{2}{|c|}{$\mathrm{DFC}_{4}(\%)$} & \multicolumn{2}{|c|}{$\mathrm{DFC}_{5}(\%)$} \\
\hline iRR & $-0,21$ & 0,14 & $-0,30$ & $0,03^{*}$ & $-0,40$ & $0,006^{*}$ & $-0,51$ & $0,0001^{*}$ & $-0,59$ & $0,0001^{*}$ \\
\hline Média (ms) & 0,25 & 0,08 & 0,37 & $0,009^{*}$ & 0,47 & $0,001^{*}$ & 0,57 & $0,0001^{*}$ & 0,64 & $0,0001^{*}$ \\
\hline Desvio padrão (ms) & $-0,11$ & 0,46 & $-0,06$ & 0,65 & 0,04 & 0,79 & 0,22 & 0,13 & 0,14 & 0,34 \\
\hline pNN50 (\%) & 0,04 & 0,78 & 0,08 & 0,57 & 0,14 & 0,34 & 0,25 & 0,09 & 0,31 & $0,03^{*}$ \\
\hline r-MSSD (ms) & 0,02 & 0,85 & 0,07 & 0,64 & 0,13 & 0,38 & 0,27 & 0,06 & 0,28 & $0,05^{*}$ \\
\hline
\end{tabular}

Correlação de Spearman. $\mathrm{DFC}_{1}$ a $\mathrm{DFC}_{5}$ : decremento relativo da frequência cardíaca obtidos do primeiro ao quinto minuto de recuperação após o teste de esforço 
Anexo IV-b:Correlação $(\mathrm{n}=46)$ entre os diversos índices temporais das séries de intervalo RR na posição ortostática previamente ao esforço com o decremento relativo das frequências cardíacas obtidas no período de recuperação ativa após o esforço

\begin{tabular}{|c|c|c|c|c|c|c|c|c|c|c|}
\hline & \multicolumn{2}{|c|}{$\mathrm{DFC}_{1}(\%)$} & \multicolumn{2}{|c|}{$\mathrm{DFC}_{2}(\%)$} & \multicolumn{2}{|c|}{$\mathrm{DFC}_{3}(\%)$} & \multicolumn{2}{|c|}{$\mathrm{DFC}_{4}(\%)$} & \multicolumn{2}{|c|}{$\mathrm{DFC}_{5}(\%)$} \\
\hline iRR & $-0,21$ & 0,15 & $-0,27$ & 0,06 & $-0,35$ & $0,01 *$ & $-0,30$ & $0,03 *$ & $-0,33$ & $0,02 *$ \\
\hline Média (ms) & 0,22 & 0,13 & 0,34 & $0,01 *$ & 0,45 & $0,002 *$ & 0,50 & $0,0001 *$ & 0,58 & $0,0001 *$ \\
\hline Desvio padrão (ms) & 0,12 & 0,39 & 0,11 & 0,43 & 0,24 & 0,10 & 0,24 & 0,10 & 0,30 & $0,03^{*}$ \\
\hline pNN50 (\%) & 0,05 & 0,72 & 0,03 & 0,82 & 0,15 & 0,32 & 0,15 & 0,29 & 0,19 & 0,20 \\
\hline r-MSSD (ms) & 0,05 & 0,73 & 0,05 & 0,73 & 0,15 & 0,31 & 0,18 & 0,22 & 0,20 & 0,17 \\
\hline
\end{tabular}

Correlação de Spearman. $\mathrm{DFC}_{1}$ a $\mathrm{DFC}_{5}$ : decremento relativo da frequência cardíaca obtidos do primeiro ao quinto minuto de recuperação após o teste de esforço 
Anexo IV-c: Correlação $(n=46)$ entre a variação relativa dos diversos índices temporais das séries de intervalo RR na transição da posição supina para posição ortostática e o decremento relativo das frequências cardíacas obtidas no período de recuperação ativa após o esforço

\begin{tabular}{|c|c|c|c|c|c|c|c|c|c|c|}
\hline & \multicolumn{2}{|c|}{$\operatorname{DFC}_{1}(\%)$} & \multicolumn{2}{|c|}{$\mathrm{DFC}_{2}(\%)$} & \multicolumn{2}{|c|}{$\mathrm{DFC}_{3}(\%)$} & \multicolumn{2}{|c|}{$\mathrm{DFC}_{4}(\%)$} & \multicolumn{2}{|c|}{$\mathrm{DFC}_{5}(\%)$} \\
\hline$\Delta \mathbf{i R R}$ & $-0,05$ & 0,72 & $-0,03$ & 0,82 & 0,002 & 0,99 & 0,08 & 0,56 & 0,1 & 0,47 \\
\hline$\Delta$ Média (ms) & $-0,005$ & 0,97 & $-0,03$ & 0,84 & $-0,008$ & 0,95 & 0,02 & 0,85 & 0,03 & 0,81 \\
\hline$\Delta$ Desvio padrão (ms) & $-0,24$ & 0,1 & $-0,24$ & 0,09 & $-0,24$ & 0,1 & $-0,27$ & 0,06 & $-0,25$ & 0,1 \\
\hline$\Delta$ pNN50 (\%) & $-0,03$ & 0,83 & 0,06 & 0,85 & $-0,07$ & 0,61 & $-0,03$ & 0,82 & $-0,08$ & 0,56 \\
\hline$\Delta \mathbf{r}-\operatorname{MSSD}(\mathbf{m s})$ & $-0,06$ & 0,68 & $-0,004$ & 0,97 & $-0,05$ & 0,71 & 0,03 & 0,80 & 0,03 & 0,81 \\
\hline
\end{tabular}

Correlação de Spearman. $\Delta$ : diferença relativa entre os valores obtidos nas posições supina e ortostática; $\mathrm{DFC}_{1}$ a $\mathrm{DFC}_{5}$ : decremento relativo da frequência cardíaca obtidos do primeiro ao quinto minuto de recuperação após o teste de esforço 
Anexo IV-d: Correlação (n=46) entre os diversos índices espectrais das séries de intervalo RR na posição supina previamente ao esforço com o decremento relativo das frequências cardíacas obtidas no período de recuperação ativa após o esforço

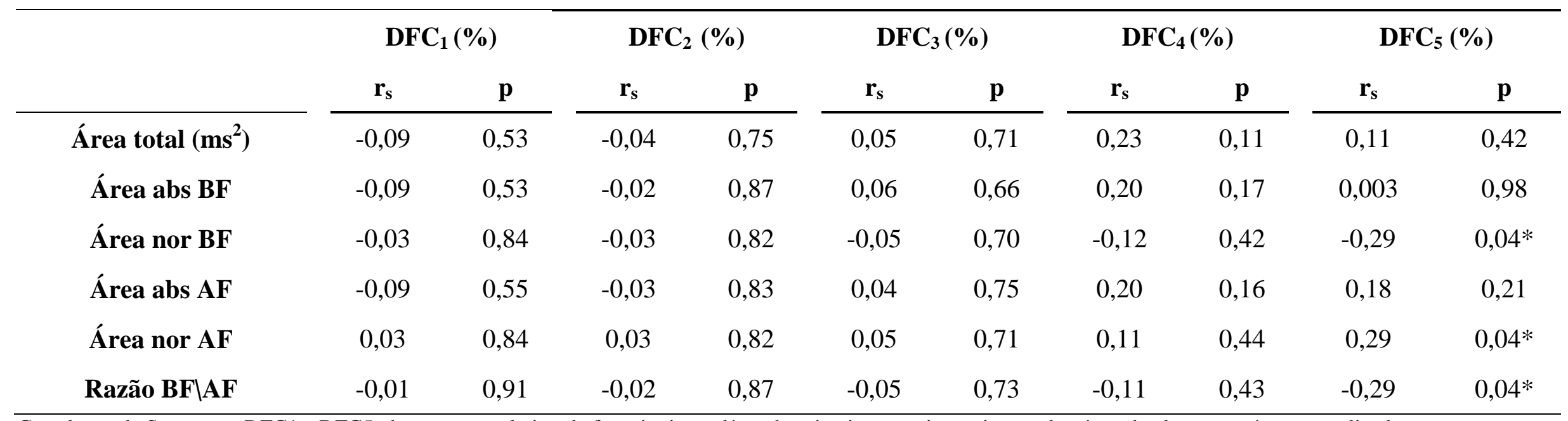

Correlação de Spearman. DFC1 a DFC5: decremento relativo da frequência cardíaca do primeiro ao quinto minuto; abs: área absoluta; nor: área normalizada 
Anexo IV-e: Correlação $(n=46)$ entre os diversos índices espectrais das séries de intervalo RR na posição ortostática previamente ao esforço com o decremento relativo das frequências cardíacas obtidas no período de recuperação ativa após o esforço

\begin{tabular}{|c|c|c|c|c|c|c|c|c|c|c|}
\hline & \multicolumn{2}{|c|}{$\mathrm{DFC}_{1}(\%)$} & \multicolumn{2}{|c|}{$\mathrm{DFC}_{2}(\%)$} & \multicolumn{2}{|c|}{$\mathrm{DFC}_{3}(\%)$} & \multicolumn{2}{|c|}{$\mathrm{DFC}_{4}(\%)$} & \multicolumn{2}{|c|}{$\mathrm{DFC}_{5}(\%)$} \\
\hline Área total $\left(\mathrm{ms}^{2}\right)$ & 0,09 & 0,51 & 0,08 & 0,56 & 0,22 & 0,12 & 0,28 & $0,05 *$ & 0,33 & 0,02 \\
\hline Área abs BF & $-0,05$ & 0,71 & $-0,001$ & 0,99 & 0,14 & 0,33 & 0,17 & 0,24 & 0,16 & 0,28 \\
\hline Área nor BF & $-0,06$ & 0,66 & 0,12 & 0,40 & 0,11 & 0,45 & 0,07 & 0,63 & 0,04 & 0,75 \\
\hline Área nor AF & 0,06 & 0,66 & $-0,12$ & 0,40 & $-0,10$ & 0,48 & $-0,07$ & 0,63 & $-0,04$ & 0,78 \\
\hline Razão BF\AF & $-0,05$ & 0,69 & 0,13 & 0,63 & 0,11 & 0,44 & 0,07 & 0,62 & 0,04 & 0,76 \\
\hline
\end{tabular}

Correlação de Spearman. DFC1 a DFC5: decremento relativo da frequência cardíaca do primeiro ao quinto minuto; abs: área absoluta; nor: área normalizada 
Anexo IV-f: Correlação $(n=46)$ entre a variação relativa dos diversos índices espectrais das séries de intervalo RR na transição da posição supina para posição ortostática e o decremento relativo das frequências cardíacas obtidas no período de recuperação ativa após o esforço

\begin{tabular}{|c|c|c|c|c|c|c|c|c|c|c|}
\hline & \multicolumn{2}{|c|}{$\mathrm{DFC}_{1}(\%)$} & \multicolumn{2}{|c|}{$\mathrm{DFC}_{2}(\%)$} & \multicolumn{2}{|c|}{$\mathrm{DFC}_{3}(\%)$} & \multicolumn{2}{|c|}{$\mathrm{DFC}_{4}(\%)$} & \multicolumn{2}{|c|}{$\mathrm{DFC}_{5}(\%)$} \\
\hline$\Delta$ Área total $\left(\mathrm{ms}^{2}\right)$ & $-0,04$ & 0,08 & $-0,08$ & 0,57 & $-0,08$ & 0,58 & $-0,14$ & 0,35 & $-0,03$ & 0,82 \\
\hline$\Delta$ Área abs BF & $-0,05$ & 0,73 & 0,07 & 0,61 & 0,05 & 0,74 & 0,02 & 0,88 & 0,12 & 0,41 \\
\hline$\Delta$ Área nor BF & 0,01 & 0,93 & 0,08 & 0,57 & 0,10 & 0,47 & 0,16 & 0,26 & $0,36^{*}$ & 0,01 \\
\hline$\Delta$ Área nor AF & $-0,02$ & 0,85 & 0,13 & 0,37 & 0,15 & 0,31 & 0,19 & 0,19 & 0,25 & 0,08 \\
\hline$\Delta$ Razão BF\AF & $-0,06$ & 0,69 & 0,08 & 0,58 & 0,11 & 0,44 & 0,19 & 0,18 & $0,32 *$ & 0,02 \\
\hline
\end{tabular}


Anexo IV-g: Correlação $(n=46)$ entre os diversos índices tempo-frequenciais das séries de intervalo RR na posição supina previamente ao esforço com o decremento relativo das frequências cardíacas obtidas no período de recuperação ativa após o esforço

\begin{tabular}{|c|c|c|c|c|c|c|c|c|c|c|}
\hline & \multicolumn{2}{|c|}{$\mathrm{DFC}_{1}(\%)$} & \multicolumn{2}{|c|}{$\mathrm{DFC}_{2}(\%)$} & \multicolumn{2}{|c|}{$\mathrm{DFC}_{3}(\%)$} & \multicolumn{2}{|c|}{$\mathrm{DFC}_{4}(\%)$} & \multicolumn{2}{|c|}{$\mathrm{DFC}_{5}(\%)$} \\
\hline Grau & $-0,11$ & 0,43 & 0,006 & 0,97 & 0,11 & 0,44 & 0,17 & 0,24 & 0,11 & 0,46 \\
\hline Natureza & $-0,1$ & 0,5 & $-0,12$ & 0,42 & $-0,11$ & 0,46 & $-0,16$ & 0,28 & $-0,27$ & 0,06 \\
\hline Área razão > 1 & $-0,05$ & 0,70 & $-0,08$ & 0,58 & $-0,11$ & 0,44 & $-0,12$ & 0,41 & $-0,23$ & 0,11 \\
\hline CV da razão & 0,28 & 0,10 & 0,23 & 0,18 & 0,19 & 0,26 & $-0,05$ & 0,76 & $-0,03$ & 0,84 \\
\hline
\end{tabular}

Correlação de Spearman. DFC1 a DFC5: decremento relativo da frequência cardíaca do primeiro ao quinto minuto; CV: coeficiente de variação 
Anexo IV-h: Correlação ( $\mathrm{n}=46)$ entre os diversos índices tempo-frequenciais das séries de intervalo RR na posição ortostática previamente ao esforço com o decremento relativo das frequências cardíacas obtidas no período de recuperação ativa após o esforço

\begin{tabular}{|c|c|c|c|c|c|c|c|c|c|c|}
\hline & \multicolumn{2}{|c|}{$\mathrm{DFC}_{1}(\%)$} & \multicolumn{2}{|c|}{$\mathrm{DFC}_{2}(\%)$} & \multicolumn{2}{|c|}{$\mathrm{DFC}_{3}(\%)$} & \multicolumn{2}{|c|}{$\mathrm{DFC}_{4}(\%)$} & \multicolumn{2}{|c|}{$\mathrm{DFC}_{5}(\%)$} \\
\hline Grau & 0,11 & 0,46 & 0,05 & 0,7 & 0,23 & 0,11 & 0,26 & 0,07 & 0,27 & 0,07 \\
\hline Natureza & 0,06 & 0,67 & 0,16 & 0,26 & 0,20 & 0,16 & 0,16 & 0,27 & 0,13 & 0,37 \\
\hline Área razão > 1 & 0,09 & 0,55 & 0,22 & 0,12 & 0,19 & 0,20 & 0,20 & 0,16 & 0,17 & 0,25 \\
\hline CV da razão & 0,25 & 0,14 & 0,23 & 0,16 & 0,25 & 0,15 & 0,32 & $0,05^{*}$ & 0,28 & 0,09 \\
\hline
\end{tabular}

Correlação de Spearman. DFC1 a DFC5: decremento relativo da frequência cardíaca do primeiro ao quinto minuto; CV: coeficiente de variação 
Anexo IV-i: Correlação $(n=46)$ entre os diversos índices tempo-frequenciais das séries de intervalo RR na transição da posição supina para posição ortostática previamente ao esforço e o decremento relativo das frequências cardíacas obtidas no período de recuperação ativa após o esforço

\begin{tabular}{|c|c|c|c|c|c|c|c|c|c|c|}
\hline & \multicolumn{2}{|c|}{$\mathrm{DFC}_{1}(\%)$} & \multicolumn{2}{|c|}{$\mathrm{DFC}_{2}(\%)$} & \multicolumn{2}{|c|}{$\mathrm{DFC}_{3}(\%)$} & \multicolumn{2}{|c|}{$\mathrm{DFC}_{4}(\%)$} & \multicolumn{2}{|c|}{$\mathrm{DFC}_{5}(\%)$} \\
\hline$\Delta$ Grau & $-0,02$ & 0,85 & $-0,19$ & 0,20 & $-0,17$ & 0,25 & $-0,11$ & 0,43 & $-0,13$ & 0,36 \\
\hline$\Delta$ Natureza & 0,14 & 0,34 & 0,25 & 0,08 & 0,27 & 0,07 & 0,25 & 0,08 & 0,37 & $0,01^{*}$ \\
\hline$\Delta$ Área razão > 1 & 0,21 & 0,14 & 0,26 & 0,07 & 0,28 & $0,05^{*}$ & 0,30 & $0,04 *$ & 0,41 & $0,004^{*}$ \\
\hline$\Delta C V$ da razão & $-0,16$ & 0,33 & $-0,10$ & 0,53 & $-0,12$ & 0,47 & 0,09 & 0,60 & 0,05 & 0,76 \\
\hline
\end{tabular}

Correlação de Spearman. $\mathrm{DFC}_{1}$ a $\mathrm{DFC}_{5}$ : decremento relativo da frequência cardíaca obtidos do primeiro ao quinto minuto de recuperação após o teste de esforço; $\mathrm{CV}$ : coeficiente de recuperação; $\Delta$ : diferença relativa entre os valores obtidos nas posições supina e ortostática 
Anexo IV-j: Correlação $(n=46)$ entre os diversos índices de Poincaré na posição supina previamente ao esforço com o decremento relativo das frequências cardíacas obtidas no período de recuperação ativa após o esforço

\begin{tabular}{|c|c|c|c|c|c|c|c|c|c|c|}
\hline & \multicolumn{2}{|c|}{$\mathrm{DFC}_{1}(\%)$} & \multicolumn{2}{|c|}{$\mathrm{DFC}_{2}(\%)$} & \multicolumn{2}{|c|}{$\mathrm{DFC}_{3}(\%)$} & \multicolumn{2}{|c|}{$\mathrm{DFC}_{4}(\%)$} & \multicolumn{2}{|c|}{$\mathrm{DFC}_{5}(\%)$} \\
\hline & $\mathbf{r}_{\mathrm{s}}$ & $\mathbf{p}$ & $\mathbf{r}_{\mathrm{s}}$ & $\mathbf{p}$ & $\mathbf{r}_{\mathrm{s}}$ & $\mathbf{p}$ & $\mathbf{r}_{\mathrm{s}}$ & $\mathbf{p}$ & $\mathbf{r}_{\mathrm{s}}$ & $\mathbf{p}$ \\
\hline $\mathbf{N}^{\circ}$ de pontos & $-0,17$ & 0,25 & $-0,16$ & 0,07 & $-0,38$ & $0,008^{*}$ & $-0,48$ & $0,001^{*}$ & $-0,58$ & $0,0001^{*}$ \\
\hline Centróide (ms) & 0,25 & 0,08 & 0,37 & $0,01 *$ & 0,47 & $0,001 *$ & 0,56 & $0,0001 *$ & 0,63 & $0,0001 *$ \\
\hline SD1 & 0,05 & 0,69 & 0,09 & 0,54 & 0,13 & 0,38 & 0,25 & 0,09 & 0,25 & 0,09 \\
\hline SD2 & $-0,09$ & 0,54 & $-0,03$ & 0,82 & 0,08 & 0,59 & 0,23 & 0,11 & 0,08 & 0,56 \\
\hline Razão SD1/SD2 & 0,19 & 0,19 & 0,17 & 0,25 & 0,14 & 0,33 & 0,16 & 0,28 & 0,29 & $0,05^{*}$ \\
\hline Área da elipse (ms2) & $-0,004$ & 0,97 & 0,05 & 0,7 & 0,13 & 0,35 & 0,29 & $0,05^{*}$ & 0,21 & 0,15 \\
\hline Coeficiente de correlação & $-0,24$ & 0,09 & $-0,27$ & 0,06 & $-0,23$ & 0,11 & $-0,22$ & 0,13 & $-0,35$ & $0,01 *$ \\
\hline
\end{tabular}


Anexo IV-k: Correlação $(\mathrm{n}=46)$ entre os diversos índices de Poincaré na posição ortostática previamente ao esforço com o decremento relativo das frequências cardíacas obtidas no período de recuperação ativa após o esforço

\begin{tabular}{|c|c|c|c|c|c|c|c|c|c|c|}
\hline & \multicolumn{2}{|c|}{$\mathrm{DFC}_{1}(\%)$} & \multicolumn{2}{|c|}{$\mathrm{DFC}_{2}(\%)$} & \multicolumn{2}{|c|}{$\mathrm{DFC}_{3}(\%)$} & \multicolumn{2}{|c|}{$\mathrm{DFC}_{4}(\%)$} & \multicolumn{2}{|c|}{$\mathrm{DFC}_{5}(\%)$} \\
\hline $\mathbf{N}^{\circ}$ de pontos & $-0,06$ & 0,66 & $-0,17$ & 0,25 & $-0,27$ & 0,06 & $-0,12$ & 0,41 & $-0,20$ & 0,16 \\
\hline Centróide (ms) & 0,21 & 0,15 & 0,33 & $0,02 *$ & 0,44 & $0,002 *$ & 0,50 & $0,0001 *$ & 0,57 & $0,0001^{*}$ \\
\hline SD1 & 0,02 & 0,89 & 0,01 & 0,92 & 0,11 & 0,45 & 0,15 & 0,31 & 0,17 & 0,23 \\
\hline Razão SD1/SD2 & $-0,12$ & 0,42 & $-0,11$ & 0,43 & $-0,06$ & 0,65 & 0,01 & 0,89 & $-0,01$ & 0,91 \\
\hline Área da elipse (ms2) & 0,06 & 0,66 & 0,05 & 0,7 & 0,16 & 0,26 & 0,18 & 0,21 & 0,23 & 0,11 \\
\hline Coeficiente de correlação & 0,13 & 0,37 & 0,14 & 0,32 & 0,08 & 0,55 & 0,01 & 0,92 & 0,02 & 0,86 \\
\hline
\end{tabular}


Anexo IV-l: Correlação ( $\mathrm{n}=46)$ entre os diversos índices de Poincaré na transição da posição supina para posição ortostática previamente ao esforço e o decremento relativo das frequências cardíacas obtidas no período de recuperação ativa após o esforço

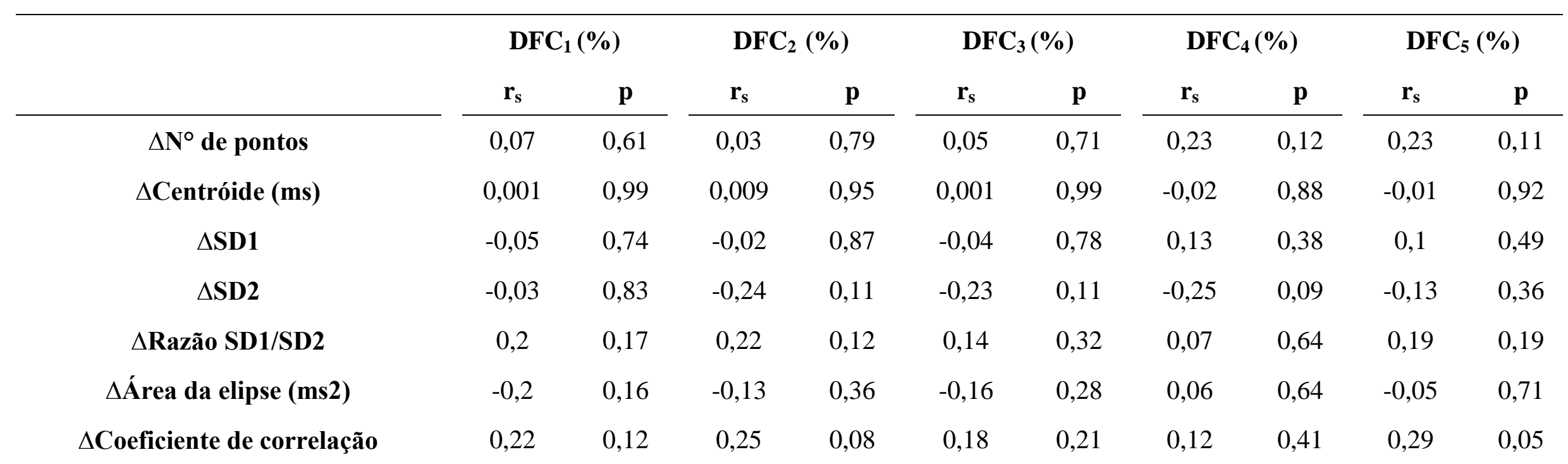

Correlação de Spearman. $\mathrm{DFC}_{1}$ a $\mathrm{DFC}_{5}$ : decrementos relativos das frequências cardíacas obtidos do primeiro ao quinto minuto de recuperação após o teste de esforço; $\Delta$ :

diferença relativa entre os valores obtidos nas posições supina e ortostática 
